\title{
WIND TURBINE AERODYNAMICS RESEARCH NEEDS ASSESSMENT
}

January 1986

Prepared by:

F. S. Stoddard, Principal Investigator

B. K. Porter, Project Coordinator

Washington Consulting Group

Washington, D.C. 20006

Contract No. DE-AC01-85ER30075

U.S. Department of Energy

Office of Energy Research

Office of Program Analysis 
Cover photograph furnished courtesy of Cermak/Peterka \& Associates Inc.,

Fort Collins, Colorado, March 1986. 


\section{DISCLAIMER}

This report was prepared as an account of work sponsored by an agency of the United States Government. Neither the United States Government nor any agency thereof, nor any of their employees, make any warranty, express or implied, or assumes any legal liability or responsibility for the accuracy, completeness, or usefulness of any information, apparatus, product, or process disclosed, or represents that its use would not infringe privately owned rights. Reference herein to any specific commercial product, process, or service by trade name, trademark, manufacturer, or otherwise does not necessarily constitute or imply its endorsement, recommendation, or favoring by the United States Government or any agency thereof. The views and opinions of authors expressed herein do not necessarily state or reflect those of the United States Government or any agency thereof. 


\section{DISCLAIMER}

Portions of this document may be illegible in electronic image products. Images are produced from the best available original document. 


\section{TABLE OF CONTENTS}

Executive Summary $\ldots \ldots \ldots \ldots \ldots \ldots \ldots \ldots \ldots \ldots \ldots \ldots \ldots$ iv

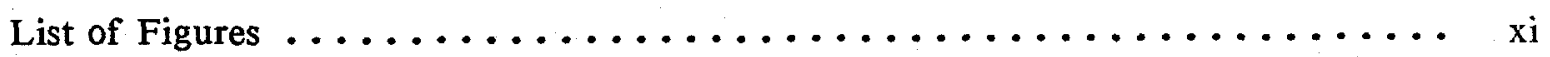

Introduction $\ldots \ldots \ldots \ldots \ldots \ldots \ldots \ldots \ldots \ldots \ldots \ldots \ldots \ldots \ldots \ldots \ldots \ldots \ldots$

\section{CHAPTERS:}

1. Technical Assessment Panel and Procedure $\ldots \ldots \ldots \ldots \ldots \ldots \ldots \ldots \ldots$

2. Research Needs Assessment Methodology .................... 4

3. Wind Turbine Aerodynamic Study Areas $\quad \ldots \ldots \ldots \ldots \ldots \ldots$

Airfoil and Rotor Behavior $\quad \ldots \ldots \ldots \ldots \ldots \ldots \ldots \ldots \ldots \ldots \ldots \ldots$

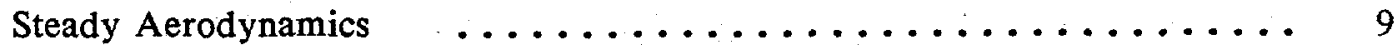

Unsteady Aerodynamics ........................ 11

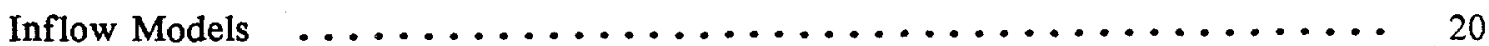

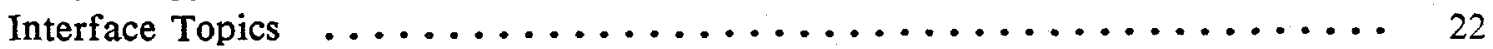

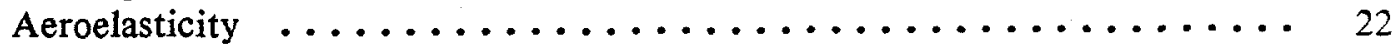

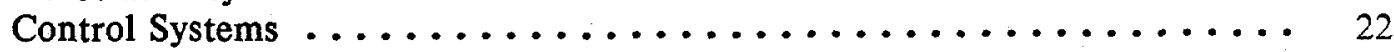

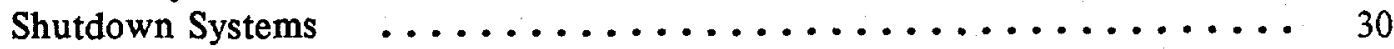

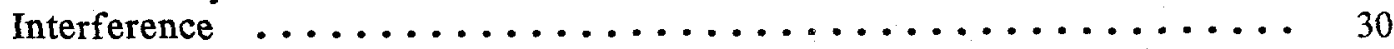

4. Wind Turbine Aerodynamic Research Needs and Priorities ........... 31

Specific Aerodynamic Research Needs by Priority $\ldots \ldots \ldots \ldots \ldots \ldots \ldots$

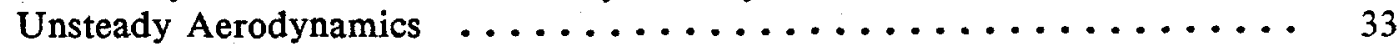

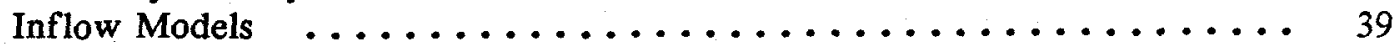

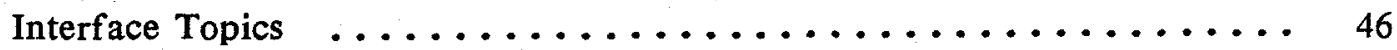

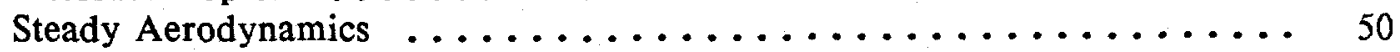

5. The Need for Basic Research $\ldots \ldots \ldots \ldots \ldots \ldots \ldots \ldots \ldots \ldots \ldots \ldots$

6. The Need for Aerometeorology $\ldots \ldots \ldots \ldots \ldots \ldots \ldots \ldots \ldots \ldots$

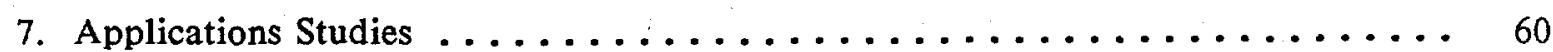

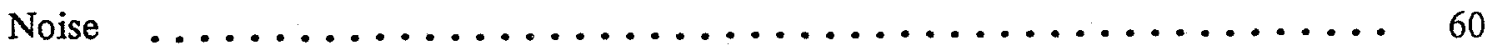

Rotor Size, Productivity, and Economy of Scale ............... 64

Variable Speed Rotors ........................... . 70

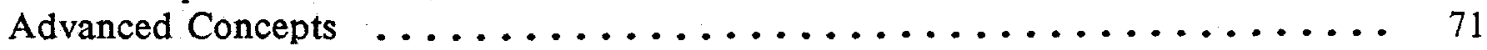

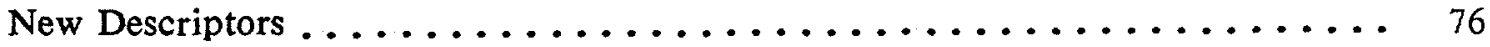

8. Recommended DOE Wind Turbine Unsteady Aerodynamics R\&D

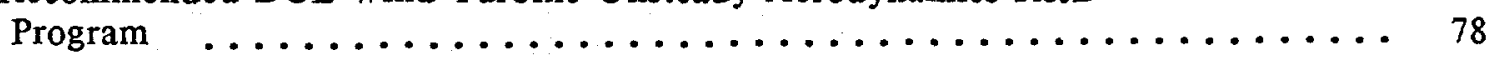

List of References $\ldots \ldots \ldots \ldots \ldots \ldots \ldots \ldots \ldots \ldots \ldots \ldots \ldots \ldots \ldots$ 


\section{APPENDICES}

1. Technical Assessment Panel and Procedures $\ldots \ldots \ldots \ldots \ldots \ldots \ldots \ldots \ldots \ldots \ldots$

2. A Tutorial: The Influence of Aerodynamics on Wind Turbine Design

Rotor Characteristics

Rotor Aerodynamic Design

100

Wind Turbine Comparison

..........

109

3. Summary of Generic Design Approaches

116

4. Wind Turbine Industry Representatives and Sites Visited, July 1985 


\section{EXECUTIVE SUMMARY}

Wind energy is a potentially viable technology, but one that now needs carefully planned research in order that pressing design problems can be solved. Wind turbine design tools must be improved for the adequate prediction of performance and reliability. This is due in part to the severe environmental demands for wind energy systems, and in part to a lack of understanding of wind turbine aerodynamics.

\section{TECHNICAL ASSESSMENT PANEL AND PROCEDURE}

The Wind Turbine Aerodynamics Technical Assessment Panel ("Panel") was formed by the Washington Consulting Group under the sponsorship of the Office of Energy Research of the U.S. Department of Energy, and was directed to define the highest priority research needs relating to the aerodynamics of wind turbines.

In addition to its own meetings and deliberations, the Panel attended a DOE-sponsored seminar on the aerodynamics of wind turbines at Sandia National Laboratory in Albuquerque, NM, in March 1985, a briefing by wind turbine industry representatives in Oakland, California, in July 1985, and conducted an inspection of a number of wind farm installations at Altamont Pass and the Boeing/PGE Mod-II wind turbine in Fairfield, California. The Panel also reviewed a substantial number of technical reports documenting the research on the aerodynamics of wind turbines.

The Panel's goal was to develop a prioritized list of wind turbine aerodynamic research needs and opportunities which could be used by the Department of Energy program management team in detailing the DOE Five-Year Wind Turbine Research Plan. The focus of the Assessment was the basic science of aerodynamics as applied to wind turbines, including all relevant phenomena, such as turbulence, dynamic stall, threedimensional effects, viscosity, wake geometry, and others which influence aerodynamic understanding and design.

The study was restricted to wind turbines that provide electrical energy compatible with the utility grid, and included both horizental axis wind turbines (HAWT) and vertical axis wind turbines (VAWT). Also, no economic constraints were imposed on the design concepts or recommendations since the focus of the investigation was purely scientific.

\section{RESEARCH NEEDS ASSESSMENT METHODOLOGY AND WIND TURBINE AERODYNAMICS STUDY AREAS}

The Panel established a method to identify the present research needs: first a hierarchy of aerodynamic study areas relevant to the engineering design of wind turbines was established. The study areas ranged from simple, steady-state, two-dimensional (2-D) airfoil studies, to the very complex stochastic representation of unsteady turbulence of the wind. Each of these was further broken down into specific subcategories, developed in order of complexity. 
The first category is the interaction between the airflow and the airfoil. This includes steady aerodynamics, on which most classical aerodynamics and most present design tools for wind turbines are based, and unsteady aerodynamics, which is concerned with airfoil and rotor behavior under time-varying conditions.

The second major aerodynamic topic is the inflow to the rotor, which consists of:

$$
\begin{aligned}
& \text { wind speed and direction } \\
& \text { fluctuations, } \\
& \text { terrain-induced fluctuations, } \\
& \text { and } \\
& \text { interference-induced } \\
& \text { fluctuations, }
\end{aligned}
$$

all of which are unsteady.

In the third and last category of aerodynamic study are interface topics, for which the aerodynamics plays a major role through the unsteady airloading, but which depend also on other disciplines. These are aeroelasticity, control systems, shutdown systems, and interference.

Next, having established a framework of topics for discussion, the Panel chose seven generic design approaches for wind turbines which are the most significant and in the Panel's view, represent the best potential for improving the cost and reliability of wind energy systems.

These were:
- Stall control
o Pitch control
- Variable speed HAWT
- Darrieus VAWT
- Straight blade VAWT
- High tip speed HAWT
- Free Yaw HAWT

Each concept was then discussed and evaluated for each aerodynamic topic, and a numerical research opportunity priority was established for each.

\section{WIND TURBINE AERODYNAMIC RESEARCH NEEDS AND PRIORITIES}

The highest priority research was identified as those topics which are common to all design approaches, that cut across design and configuration boundaries, and will enhance those approaches and others yet to be identified.

The Panel agreed that the following three subject areas and specific subdisciplines are the highest priority aerodynamic research areas and opportunities for future development of the wind-power industry:

\section{Unsteady Aerodynamics}

Dynamic Stall Understanding--

Develop an understanding of the 2-D and 3-D hysteresis effects of dynamic stall.

Testing Methodology for Unsteady Flow.--

Stimulate complex unsteady flows in wind tunnel and total system (field) tests in order to exploit dynamic stall effects.

Airfoil Development Studies for Unsteady Flow--

Initiate a design process which will yield airfoils specifically suited for unsteady flow, and which considers the significance of: 
o dynamic stall repeatability and insensivity

o airfoil roughness

o delayed stall and sof $t$ stall

o performance and prediction

o rotor stability; and

- airfoil control devices.

Wind Inflow Models: The Development of Aerometeorology

Establish a new study termed aerometeorology, which will attempt to develop realistic inflow models for the assessment of unsteady effects, including the specific cases of:

- unsteady, uniform inflow-or representation of the uniform gust front;

o unsteady nonuniform inflow-or representation of frozen turbulence;

o steady, nonuniform inflow-. or representation of steady inflow fronts; and

o stochastic inflow-or representation of inflow in the frequency/wave number domain.

\section{Interface Topics}

Aeroelasticity--

Develop a methodology for defining the unsteady airloads which must be included in the structural dynamic models.

\section{Control Systems--}

Investigate the performance and reliability benefits to be obtained with external control actions, such as ailerons, in steady and unsteady flow.
Wake Model Interference Development--

Develop and verify a model for the structure and decay of the unsteady wind turbine rotor wake.

\section{Component Wake Interference} Development--

Develop and verify models for the structure and decay of the unsteady wakes caused by turbine components such as towers.

Shutdown (Emergency) Systems--

Develop an understanding of the behavior of airfoils under extreme conditions, and at very high angles of attack, such as a moving aileron in separated flow.

In addition, further steady-state aerodynamics studies should be continued in order to provide a suitable data base for the above investigations and lend insight into the new physical and mathematical models and design tools which must be used.

\section{Steady Aerodynamics}

\section{Three-Dimensional Flow--}

Assess the degree of 3-D or spanwise flow which occurs under typical conditions, and relate that to the inflow and turbine wake geometry.

\section{Wake Modeling--}

Develop and verify a model of the turbine vortex wake geometry suitable for performance and stability studies. 
Effect of Roughness in Steady State--

Develop an understanding of environmentally-induced airfoil surface roughness on airfoil transition and separation in steady flow.

\section{Airfoil Mechanisms-- \\ Investigate the effect of airfoil mechanisms, such as vortex generators, on delaying turbine rotor stall and separation.}

Two-Dimensional Airfoil Development--

Develop a verified methodology for tailored wind turbine airfoil design in steady state to establish a database for future unsteady airfoil design.

Testing Methodology for Steady Flow--

Continue steady-state wind turbine testing in wind tunnels and in the field, to acquire long-term performance data and to investigate the benefits of new airfoils.

\section{THE NEEDS FOR BASIC RESEARCH AND AEROMETEOROLOGY}

The Panel determined that the major work should be in unsteady aerodynamics and rotor inflow and recommends that the emphasis be on basic research rather than on applied research. The basic research which is called for here must establish a technology base strong enough so that design decisions can be made with confidence and not by trial and error as is of ten the case now. The Panel believes that very signif icant improvement in the perfor- mance, reliability, and economics of wind turbines is possible given a proper unsteady aerodynamics technology base. This basic research program should include a strong experimental emphasis from the beginning.

The Panel also calls for the development of a new branch of aerodynamic study, termed aerometeorology, which combines the unsteady aerodynamics of the wind turbine design community and the unsteady physics of the meteorologists. The center of expertise for the study of unsteady rotor inflow is the atmospheric physics community. However, sufficient guidelines do not exist since the wind turbine community cannot yet adequately assess airfoil response to fluctuations. A better understanding of unsteady aerodynamics and new attempts to relate the extensive body of micrometeorological data on turbulence structure to engineering applications are needed. Therefore, the basic goal of aerometeorology is:

To define a disciplinary area of collaboration between the wind turbine engineering community and the micrometeorology community, both experimental and theoretical.

In aerometeorology, the emphasis will be on representing realistic unsteady inflows to the aerodynamicists who will be assessing various wind turbine design concepts. For example, two of the immediate projects that could be undertaken by aerometeorologists would be the determination of effective rotor inflow length scales and micrositing, or the representation of terrain interference inflow effects on distributions of mean inflow velocities. 


\section{DIFFICULTY OF THE PROBLEM}

The nature of the basic research goals makes it difficult to estimate the time or cost needed for the recommended research study areas. Innovations and discovery of new approaches and solutions will modify any set of strategic goals or milestones adopted. The most that can be done is to define the higher payoff directions which are clear now, and will eventually bear fruit. We expect that these directions will also change in the future as knowledge is gained.

This course of action is not a short-term strategy, but a major patient, continuing long-term commitment. It depends on substantial directed resources and talent that carefully constructs theoretical programs coupled with strong experimental support, and is allowed to gain momentum over a span of years, probably decades.

\section{APPLICATIONS STUDIES}

Additional studies that merit attention are in the area of applications: noise, rotor economy of scale, advanced concepts, and new descriptors.

Rotor noise, especially blade tip noise due to high tip speed, will be an important siting criterion. The capability to predict aerodynamic noise is well in hand provided the unsteady airloads can be determined. For wind turbines, the technology need is to provide these unsteady loads to the prediction programs.
An economy of scale is possible with larger wind turbine rotors. However, it is clear that the effects

are not simple. Clearly, more needs to be known about the unsteady performance of large rotors and the representation of the unsteady inflow, before a definitive conclusion can be made about economy of scale of large wind turbines.

A variable speed rotor which operates at constant tip speed ratio is attractive since the rotor operates at maximum power coefficient (efficiency) when it is at constant tip speed ratio. Another advantage that is not so easily quantified is the equilibrium of operation. Simply stated, for constant tip speed ratio the aerodynamic and inertial rotor blade loads are in balance for all inflow wind speeds.

A technology applications need is to determine the benefits of this.

There is potential for large improvement in output of wind turbines by using advanced aerodynamic concepts. The ducted turbine is one example of a family of advanced concepts all of which have the potential to exceed the Betz limit (based on rotor swept area) on power coefficient $(0.5926)$, which is generally held to be the ideal maximum for conventional rotors.

Lastly, new descriptors or nondimensional parameters need to be adopted which are relevant to wind turbines, both in the aerodynamics and in the system analysis. The most valuable historical use of non-dimensional aerodynamic quantities has been in the scaling and similarity 
studies necessary for small-scale controlled testing. However, these parameters are also useful in comparing various design concepts for performance reliability.

\section{RECOMMENDED WIND TURBINE AERODYNAMICS R\&D PROGRAM}

A national R\&D program for implementing the wind turbine unsteady aerodynamics research needs put forth in this Report can occur within the present framework of the current DOE wind energy program as stated in the current Five-Year Wind Energy Program Plan. The three major parts to the present DOE program are:

1. to sponsor basic research,

2. to conduct research on advanced components and systems, and

3. to transfer the research results to industry.

The current DOE plan thus places increased emphasis on improved understanding of the basic physical phenomena involved in converting the wind to useful energy.

The (draft) November 1985 Revised Comprehensive Program Management Plan goes further in this regard to establish specific objectives:

- "Improve the understanding of fundamental sources of wind variability--local windflow variability and shear, and turbineto-turbine interactions."

- "Increase basic understanding of the interactions between wind input and structural response and the resulting effects on performance and loads."

- "Investigate, through generic, proof-of-concept activities, the potential for improved performance using advanced components and subsystems."

o "Develop an advanced multimegawatt wind turbine.

The Panel Assessment has provided the proper focus for specific tasks which meet the first two objectives above, namely improving the understanding of fundamental sources of wind variability, and increasing the basic understanding of the interactions between the wind, the structural response, and the performance of wind energy systems. Further, the recommended work will eventually result in adequate and confident design tools, thus permitting major improvements in productivity and reliability of future wind energy systems.

The specific goals of the individual investigations cannot be described in detail until additional work is done. The most that can be said here is that an effective plan will incorporate the following aspects:

- Stressing basic research,

- Striving for better understanding of the physical phenomena,

- Maintaining a patient, long-term attitude,

o Keeping distance from the commercial uses, and

o Coordinating knowledge from disciplines related to aerodynamics and affecting wind turbine design.

The bulk of the $R \& D$ investigations should ideally occur at a facility which would include both analytical and experimental researchers to permit frequent and informal communication. In-house funding and technical staffing should allow a wide degree of investigative freedom, but care should be taken to discour- 
age proprietary, closed, or near-term commercializable projects.

A first-rate wind tunnel with unsteady flow and turbulencegenerating capability is needed.

Also, a sophisticated data-processing center is required to permit accurate storage of data, generation of turbulence statistics, verification of analytical codes, and manipulation of large datasets. An associated field installation should have rotors with full aerodynamic and dynamic instrumentation.

Given that the above will be very difficult to achieve, the Panel recommends a next-best approach of subcontracting for the wind tunnel, and keeping the rest of the effort together at a common facility. 


\section{LIST OF FIGURES}

1. Aerodynamic Study Areas for Wind Turbines $\ldots \ldots \ldots \ldots \ldots \ldots$

2. Wind Turbine Rotor Generic Approaches $\ldots \ldots \ldots \ldots \ldots \ldots$

3. Wind Turbine Research Needs and Priorities $\ldots \ldots \ldots \ldots \ldots \ldots$

4. Airfoil Stall Selection Chart $[4] \quad \ldots \ldots \ldots \ldots \ldots \ldots \ldots \ldots \ldots \ldots \ldots$

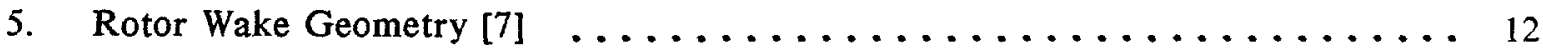

6. Separation Control Devices $[8] \ldots \ldots \ldots \ldots \ldots \ldots \ldots \ldots \ldots \ldots$

7. Dynamic Stall of NACA 0012 Airfoil [9] ................. 14

8. Delayed Stall and Soft Stall $\ldots \ldots \ldots \ldots \ldots \ldots \ldots \ldots \ldots \ldots \ldots \ldots \ldots$

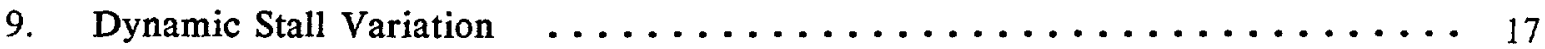

10. Airfoil Surface Roughness Effects in Steady State $[11] \quad \ldots \ldots \ldots \ldots \ldots$

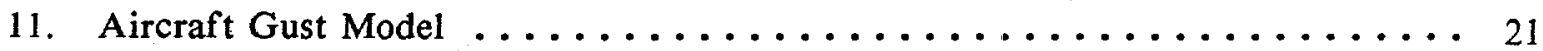

12. Wind Inflow: Uniform Front, Steady and Unsteady $\ldots \ldots \ldots \ldots \ldots$

13. Wind Inflow: Nonuniform Front, Steady $\ldots \ldots \ldots \ldots \ldots \ldots \ldots \ldots$

14. Atmospheric Inflow Fluctuations $\ldots \ldots \ldots \ldots \ldots \ldots \ldots \ldots \ldots \ldots$

15. Wind Inflow: Nonuniform Front, Unsteady $\ldots \ldots \ldots \ldots \ldots$

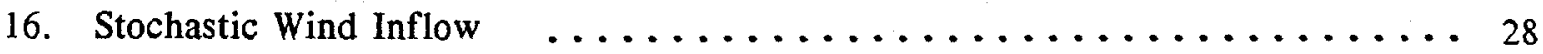

17. Wind Turbine General Control Regions . . . . . . . . . . . . . . . 29

18. High Tip Speed Rotors: Effect of Inflow Fluctuations $\ldots \ldots \ldots \ldots \ldots$

19. Effect of Roughness on Power Curve $\ldots \ldots \ldots \ldots \ldots \ldots \ldots$

20. Yaw Stability $\ldots \ldots \ldots \ldots \ldots \ldots \ldots \ldots \ldots \ldots \ldots \ldots \ldots \ldots \ldots \ldots$

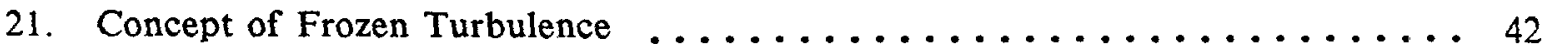

22. Total System (Field) Test Arrangement $\ldots \ldots \ldots \ldots \ldots \ldots \ldots \ldots$

23. Effect of Shear Front on Angle-of-Attack Distribution $\ldots \ldots \ldots \ldots \ldots$

24. Rotor Harmonics in the Inflow Power Density Spectrum [28] $\ldots \ldots \ldots \ldots$ 
25. Complete Wind Inflow Representation $\ldots \ldots \ldots \ldots \ldots \ldots \ldots \ldots$

26. Pitch Control vs. Constant Tip Speed Ratio (Variable Speed) $\ldots . . . . . . .49$

27. Aerometeorology $\ldots \ldots \ldots \ldots \ldots \ldots \ldots \ldots \ldots \ldots \ldots \ldots \ldots \ldots \ldots$

28. Rotor Noise: Effect of Tip Speed [52] $\ldots \ldots \ldots \ldots \ldots \ldots \ldots \ldots \ldots$

29. Rotor Noise: Typical Comparison $[52,53] \quad \ldots \ldots \ldots \ldots \ldots \ldots \ldots \ldots \ldots$

30. Rotor Noise: Effect of Disk Loading $[52,53] \ldots \ldots \ldots \ldots \ldots \ldots$

31. Economy of Scale: Land use Efficiency and Profile Effect [54] . . . . . 65

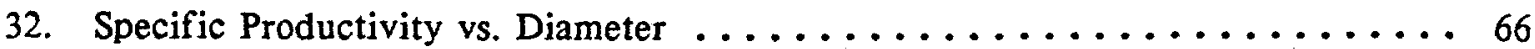

33. Wind Turbine Energy Productivity (Est.) $[55] \quad \ldots \ldots \ldots \ldots \ldots \ldots \ldots$

34. Specific Productivity vs. Rotor Area $\ldots \ldots \ldots \ldots \ldots$

35. Economy of Scale Drivers for Large Wind Turbine Rotors . . . . . . . . . . . 69

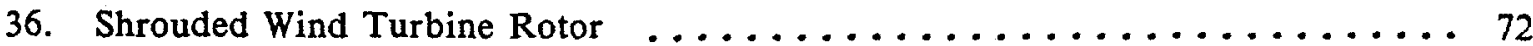

37. Average Mass Flow Increase Through an Annular Wing $[15] \ldots \ldots \ldots \ldots$

38. High Lift Coefficients from Flaps $[58] \ldots \ldots \ldots \ldots \ldots \ldots \ldots \ldots$

39. Tip Vane Rotor $[59] \quad \ldots \ldots \ldots \ldots \ldots \ldots \ldots \ldots \ldots \ldots \ldots \ldots$

A-1. Blade Element Diagrams in Various Rotor States $[31] \ldots \ldots \ldots \ldots \ldots \ldots$

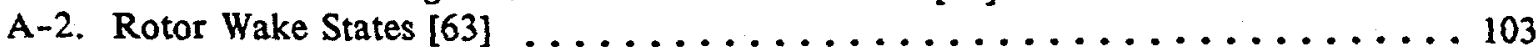

A-3. Various Coefficients vs. Tip Speed Ratio .................. 105

A-4. Dynamic Stall Events on a NACA 0012 Airfoil [Ref. 9] . . . . . . . . . . . 107

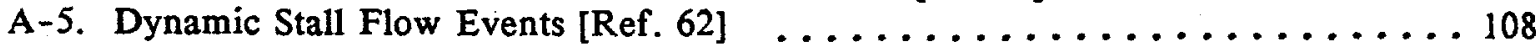

A-6. Rotor Comparison: Thrust and Power Coefficient . . . . . . . . . . 111

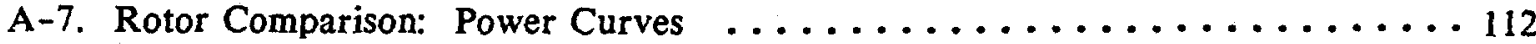

A-8. Performance of Example Pitch-Control Rotor $\ldots \ldots \ldots \ldots \ldots \ldots \ldots \ldots \ldots$

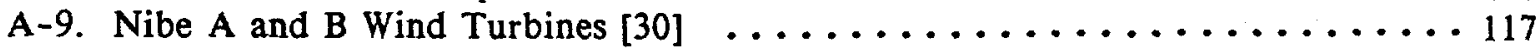

A-10. Nibe A Turbine: Stall-Induced Vibrations $[30] \ldots \ldots \ldots \ldots \ldots \ldots \ldots \ldots$

A-11. Nibe B Turbine: High Aerodynamic Braking Load [69] . . . . . . . . . 120

A-12. Darrieus Wind Turbine Rotor ..................... 122

A-13. Straight Blade Vertical Axis Wind Turbine $\ldots \ldots \ldots \ldots \ldots \ldots \ldots \ldots \ldots$ 


\section{INTRODUCTION}

\section{GOALS OF CURRENT WIND TURBINES}

The design goals for cost-effective wind turbines are simply to increase captured energy and to extend useful lifetime. Current wind turbines need a 2 to 3 factor increase in energy production, or alternatively, 2 to 3 factor decrease in cost, or a combination of both [1].

All wind turbines, both horizontal axis (HAWT) and vertical axis (VAWT), have the two resulting engineering design concerns of performance and reliability. The engineering challenge is to satisfy these two aims within the economic constraints. The economic potential of wind turbines is also thus strongly dependent on the available wind resource, the site conditions, and local competing energy cost.

\section{ROLE OF AERODYNAMICS}

A wind turbine system consists of interrelated mechanical and electrical parts, each having its own complexity and industrial practice. However, the rotor is undeniably the most complex, least understood, and most vulnerable part of the system. It is the principal component in extracting energy from the wind. It must perform its function efficiently and reliably for very long periods with little attention. Therefore, the aerodynamics of wind turbine rotors is a crucial technology area for attainment of these goals. Aerodynamics research which can improve performance and reliability is the principal subject of this Technical Needs Assessment.
Appendix 2 is a brief introduc tion to the influence of aerodynamics on wind turbine rotor design. That Section can be referred to by readers who are unfamiliar with the wind turbine or aerodynamic concepts used in the body of this Technical Assessment.

\section{WIND TURBINE AERODYNAMICS} PROBLEM

Improving wind turbine aerodynamic design requires state-of-the-art aerodynamic knowledge, and for a range of readily identified circumstances, requires knowledge not presently available. In some areas, the wind turbine problems present more severe demands than classical flight vehicle aerodynamic design. It is not an overstatement to say that some aspects of wind turbine aerodynamics are on the frontiers of fluid dynamics.

The environment of the wind turbine is more complex and more difficult to quantify than the environment for flight vehicles. Essentially all the operating time is spent in unsteady flow, with its associated potential for dynamic stall and airloading uncertainties. Extreme aerodynamic limit conditions are relatively common experiences. Wind turbines have a very wide array of possible dynamic conditions, and unlike flight vehicles, including helicopters, spend a great deal, if not a majority, of their design life in transient motions.

Wind turbine rotor response to wind fluctuations is not well under- 
stood. In normal operation, turbulent shear flow interacts with the wind turbine rotor to create unsteady flow and forces on the airfoils. The result is wind turbine system response which is complex and dynamic. Adequate response prediction is necessary for reliable structural design.

Therefore, successful wind turbine development and efficient deployment are now limited by insufficient understanding of the unsteady aerodynamics of the blades and an incomplete description of atmospheric fluctuations. This results in the inability to describe the phenomena mathematically, and then predict the aerodynamic and dynamic response. Current wind turbine design codes include significant empirical portions, and progress in the hardware has of ten been by trial and error. The greatest significance of this is the present inability to predict the blade loading and performance adequately.

\section{DEFINITION OF THE SYSTEMS RESEARCH PROBLEM AND CONTEXT OF THIS TECHNICAL ASSESSMENT}

Nevertheless, the foundations of wind turbine aerodynamics are the same as those of aerodynamic analysis and design of flight vehicles. A satisfactory physical and mathematical model is sought which reproduces all important aspects of the wind turbine behavior. The ultimate mathematical model of the flow would be based on the classical foundation of fluid mechanics (the Navier-Stokes equations), with boundary conditions providing a complete geometric description of the rotor, whose motion would be determined though a coupled solution of the structural equations. The shaft loading which determines the torque as a function of shaft speed would be included, as would be the constraints implemented by control systems. The incident inflow field would be unsteady and nonuniform, with properties particular to the expected site.

This ultimate system model is logically decomposed into three parts which interact with one another to yield the system behavior:

Structural Model: describes the geometric shape and how it moves,

Electrical Model: provides the load torque as a function of shaft speed, and

Aerodynamic Model: describes rotor response to inflow fluctuations.

The rotor aerodynamic response to inflow fluctuations is the most critical for future development of wind turbines. Such a solution of the Navier-Stokes equations for a reasonable model of a wind turbine, under a reasonable unsteady nonuniform wind environment, is well beyond the state-of-the-art. It is therefore necessary to break the aerodynamic problem down into a collection of subproblems which have some degree of tractability, so that progress can be made. It is imperative also that appropriate experimental checks be made on all of the proposed subsolutions.

The context of this Technical Assessment is the identification of this particular list of subproblems, and those which have the highest priority for future improvement in wind energy system performance and reliability. 


\section{PRESENT FEDERAL WIND TURBINE AERODYNAMICS R\&D ACTIVITY}

Wind turbine aerodynamics is just one of a number of general topics in the DOE wind turbine program, which also includes structural dynamics, advanced components, multimegawatt systems, and supporting research. Aerodynamics and atmospheric fluid dynamics, which are the subjects of this study, are currently being studied in the DOE program within the following array of subtopics:

1. Airfoils (2-D steady)

2. Unsteady Aerodynamics (2-D unsteady)

3. Unsteady Aerodynamics (3-D using some 2-D models)

4. Steady-State Rotor Performance Modeling

5. Three-Dimensional Flows

6. Rotor Performance Improvements

(steady-state)

7. Rotor Control and Braking with Ailerons

\section{Structural/Aerodynamic Interaction \\ 9. Stochastic Wind Effects}

The specific programs in these subtopics were presented by the DOE research groups, and were reviewed by the Panel, at the initial aerodynamics program review meeting and seminar at Sandia National Laboratory in March [3].

The DOE uses five laboratory/ centers around the country to accomplish these studies. Some university and subcontractor work is funded through these laboratories, and some work is done in-house. The program includes both analytical and experimental work, the bulk of the latter being done on the test-bed wind turbines which were erected in the earlier years of the DOE program (see table below). The DOE FY 1985 resource requirements for wind turbine research was $\$ 31.6$ million; the budget peaked in 1979 at $\$ 60.7$ million.

\section{LABORATORY/CENTER}

PROGRAM SCOPE TEST BED

Sandia National Lab.

NASA Lewis Res. Center

Battelle Pacific NW Lab.

Solar Energy Res. Inst. (formerly Rocky Flats)
VAWT

Large HAWT

Wind \& Siting

Small HAWT

Generic Research

And Basic Wind

Energy Science 17-meter VAWT

MOD-0 $125 \mathrm{KW}$

MOD-0A $200 \mathrm{KW}$

MOD-2 $2500 \mathrm{KW}$

None

Numerous systems up to $100 \mathrm{KW}, 15$-meter diameter at the field test site; Controlled Velocity Test Facility 
PRESENT WIND TURBINE INDUSTRY ACTIVITY

The present wind turbine industry consists of about 30 US and 20 foreign manufacturers of commercial wind turbines. The bulk of these are sized in the 10-20 meter diameter class, corresponding to roughly 25-250 KW rated output. Some are designed for the remote power, agricultural, supplemental-energy markets, but the majority are designed for the centralized utility windfarms. There is much worldwide interest at present in stand-alone, diesel-assist, decentralized turbines, but little market activity or sales in that market as yet.

The utility-connected windfarms in California are by far the largest installation of present production wind turbines. This has occurred as a result of federal and state legislation which established two favorable conditions:

1. Federal wind energy tax credit of $25 \%$ plus California's additional $25 \%$ total tax credit for commercial wind turbines (expiring in 1986).

2. Favorable utility participation in utility power purchase agreements of Qualifying Facilities under PURPA (Public Utilities Regulatory Policy Act of 1978) legislation by the largest California private utilities, Southern California Edison and Pacific Gas \& Electric Co.

In 1981 there were a total of only 142 interconnected turbines in two windfarms in the Altamont Pass, California, and they comprised the extent of the commercial deployment. By the end of 1985 there were over 10,000 utility-interconnected turbines in California representing roughly $\$ 1.5$ billion total financing through limited partnerships usually employing tax shelters. The average capacity per machine in 1981 was $50 \mathrm{KW}$, and in 1984 was $97 \mathrm{KW}$. The average annual output per operating machine was $35,000 \mathrm{KWh}$ in 1981 , and 120,000 $\mathrm{KWh}$ in 1984 . The cumulative total installed capacity is now over 1000 Megawatts in California, which represents about $2.5 \%$ of the total capacity of the state. The wind turbines have produced over 800 million Kilowatt-hours of electricity, and have long since passed the milestone of having displaced the first one million barrels of oil equivalent.

The growth of the industry has been very rapid since the enactment of the tax credits. There are at present in California three major sites (Altamont Pass, Tehachapi Pass, and San Gorgonio Pass) with over 100 windfarm projects. About half of these wind machines are from manufacturers located in foreign countries, principally Denmark, who have aggressive wind energy development and export policies.

\section{ORGANIZATION OF THIS REPORT}

Chapter 1 describes the Technical Assessment Panel, and its procedures and goals. Chapter 2 discusses the methodology adopted by the Panel for establishing the wind turbine aerodynamic research needs. The general wind turbine aerodynamic study areas are defined and described in Chapter 3. The Panel's prioritized research needs and specific recommended programs in each study area are given in Chapter 4. The need for basic reseach in the federal program is discussed in Chapter 5 , and the need for a new field of 
aerodynamic study, which the Panel has termed aerometeorology, is discussed in Chapter 6. Other engineering applications studies which are important are given in Chapter 7. Finally, aspects of the recommended federal $R \& D$ program are described in Chapter 8.

Appendix 1 contains the technical references reviewed by the Panel in the course of discussions, Appendix 2 is a short tutorial on the influence of aerodynamics on wind turbine engineering design, and Appendix 3 describes the generic wind turbine engineering concepts which were considered by the Panel. In Appendix 4 is a list of the wind industry representatives who made formal presentations, and a list of the wind turbine sites visited by the Panel. 
The Wind Turbine Aerodynamics Technical Assessment Panel ("Panel") was formed by the Washington Consulting Group under the sponsorship of the Office of Energy Research of the U.S. Department of Energy, and was directed to define the highest priority research needs relating to the aerodynamics of wind turbines. We list the Panel members below, and also describe the procedure and goals of this effort. The panel consisted of nationally-recognized aerodynamacists who have the individual and combined expertise to provide an objective and profound evaluation of wind turbine aerodynamics and the present $R \& D$ programs.

In order to acquaint the Panel members with research activities to date relating to the aerodynamics of wind turbines, the Panel attended a Department of Energy-sponsored seminar on the subject at Sandia National Labor New Mexico, March 26-28, 1985 [3], and also received a briefing by wind turbine industry representatives held in Oakland, California, July 10-12, 1985. At that meeting, the Panel inspected a number of the private wind farm installations at Altamont Pass, California, and the Boeing/PGE Mod-II wind turbine in Fairfield, California. The industry participants of the Oakland meeting and the sites visited are listed in Appendix 4.

In addition to the meetings, the Panel reviewed a number of reports including the Department of Energy Five Year Plan for Wind Energy document summarizing the
Development [1] and a DOE planning DOE-sponsored activities in the field of wind energy which were undertaken during 1984 [2]. Other technical reports documenting the research on the aerodynamics of wind turbines were made available by DOE and were reviewed (see Appendix 1).

\section{GOAL}

The Panel's goal was to develop a prioritized list of wind turbine aerodynamic research needs and opportunities which could be used by the Department of Energy program management team in refining and updating the DOE Five Year Wind Turbine Research Plan [1].

\section{FOCUS}

The focus of the Panel Assessment was the basic science of aerodynamics as applied to wind turbines, including all relevant phenomena, such as turbulence, dynamic stall, threedimensional effects, viscosity, wake geometry, and any others which influence aerodynamic understanding and design.

\section{OBJECTIVES}

The specific objectives of this Panel Assessment were to:

1. Identify and prioritize research needs and opportunities.

2. Identify those for which a government role is necessary.

3. Identify current research which is of lower priority. 


\section{DESIRED OUTPUT}

The final technical assessment report was specified to include:

1. Research needs by topic and activity.

2. Relative priority of research needs and opportunities, with rationale.

3. Options for performing needed research.

4. Cost and duration of proposed R\&D activities.

5. Activities underway deemed to be of lower priority.

\section{WORKING PLAN}

The working plan of the Panel was to develop guidelines that could be used by the Department of Energy, along with the Five Year Plan, for a wind energy technology R\&D program which would lead ultimately to a viable wind turbine industry. The assessment was to specifically look ahead in time to identify $R \& D$ areas that should be started now in order to strengthen the turbine industry in the future. The Panel was instructed to look at the present program only insofar as necessary to appreciate the complexity and scope of work to date.

\section{CRITERIA FOR PANEL SELECTION}

The individuals on this Technical Assessment Panel were chosen specifically for their range and depth of expertise in the various areas of aerodynamics. The Panel is qualified to research, describe, and plan any program in aerodynamics of any degree of sophistication or technical objective. The range of specific aerodynamic expertise represented on the Panel was determined by the needed study of wind turbines and atmospheric interaction. An additional requirement for the Panel was that none of them be presently under contract to the U.S. DOE Wind Energy Program.

\section{WIND TURBINE AERODYNAMICS} TECHINICAL ASSESSMENT PANEL

Barnes W. McCormick, Panel Chairman Department of Aerospace Engineering Penn. State University

Jewel B. Barlow

Glenn L. Martin Wind Tunnel

University of Maryland

Lawrence W. Carr

U.S. Army Aeroflightmechanics

Laboratory

NASA/Ames Research Center

Jack E. Cermak

Fluid Mechanics and Wind Engineering Colorado State University

David R. Ellis

Aircraft Division

Cessna Aircraft Company

Stan J. Miley

Department of Aerospace Engineering Texas A\&M University

John C. Wyngaard

National Center for Atmospheric

Research

\section{PROJECT TASKS}

1. Assess the State-of-the-Art of wind turbine aerodynamics research.

2. Conduct two workshops to review the research and development activities now underway. These workshops were to include responses to the research review and Panel discussion of perceived research needs and opportunities. 
3. Prepare a final report giving the Panel's recommendations and fulfilling the objectives of the technical assessment study.

\section{SCHEDULE}

Albuquerque, NM, March 25-28, 1985, DOE Wind Turbine Aerodynamics Research Program Review and Seminar

June 1985, Draft Report: "State of the Art/Expertise" Working Document reviewed by the Panel

Oakland, CA, July 10-12, 1985 Wind Turbine Industry Briefing

Altamont Pass, CA, July 12, 1985 , Wind Turbine Site Visits

Washington, DC, September 26-27, 1985, Final Panel Meeting 


\section{CHAPTER 2. RESEARCH NEEDS ASSESSMENT METHODOLOGY}

The Panel, having reviewed and gained an appreciation for the government and industry wind turbine aerodynamics research activities, decided on a method to identify the present research needs. First, a hierarchy of aerodynamic study areas was established (see Figure 1). These study areas were chosen to be relevant to the engineering design of wind turbines, and were discussed in depth by the Panel. The study areas range from simple, fundamental two-dimensional (2-D) airfoil studies, to the very complex stochastic representation of unsteady turbulence of the wind. These study areas are described in Chapter 3 , and represent the historical sequence of aerodynamics development.

Next, having established a framework of topics for discussion, the Panel chose seven generic design approaches for wind turbines, which, in the Panel's view, are the most significant and present the best potential for improving cost and reliability of wind turbines. These are described fully in Appendix 3, and are listed in Figure 2. Each concept was discussed and evaluated for each aerodynamic topic, and a numerical research opportunity priority was established for each. In this way, the particular research needs and opportunities were established for each concept. The highest priority research was then identified as those topics which were most significant and presented the most opportunity across the board. These are identified and discussed in Chapter 4.

\section{AREAS OF AERODYNAMIC STUDY}

The aerodynamic study of wind turbines was logically categorized by the Panel into three broad areas:

1. The airfoil and rotor behavior,

2. The inflow to the rotor, and

3. Interfaces with other disciplines.

Airfoil behavior is the steady and unsteady (i.e., constant and varying) response of the airfoil to changes in its flowfield. Most aircraft aerodynamics work rests on steady-state analysis, and all the present wind turbine design tools assume steady-state airflow over the rotor blade. The inflow to the rotor consists of unsteady components due to a myriad of factors including wind fluctuations, terrain effects, and interference from other aerodynamic wakes. Other wind turbine research disciplines depend intimately on the rotor response: aeroelastic analysis is both structural and aerodynamic, controls response analysis includes aerodynamic loading, and interference is the aerodynamic influence of other wakes.

\section{WIND TURBINE GENERIC APPROACHES}

The wind turbine aerodynamic research needs depend on the design approach taken. There is a large variety of rotor types, each with its own set of specif ic design requirements and characteristics, 
STEADY AERODYNAMTCS

AIRFOIL STUDIES:

TWO-DIMENSIONAL

THREE-DIMENS IONAL

EFFECT OF ROUGHNESS

AIRFOIL DEVELOPMENT

WAKE ANALYSIS

MECHANISMS :

HIGH LIFT DEVICES

SEPARATION CONTROL DEVICES

TESTING METHODOLOGY

UNSTEADY AERODYNAMICS

DYNAMIC STALL UNDERSTANDING:

TWO-DIMENSTONAL HYSTERESIS

THREE-DIMENSIONAL HYSTERESIS

AIRFOIL DEVELOPMENT STUDIES:

DELAYED STALL

SOFT STALL

REPEATABLE STALL

INSENSTTIVE STALL

EFFFCT OF ROUGHNESS

AIRFOIL DEVICES

TESTING METHODOLOGY:

WIND-TUNNEL TESTING

TOTAL SYSTEM. (FIELD) TESTING

PREDICTION OF PERFORMANCE

ROTOR STABILITY

WIND INFLOW MODELS

STEADY, UNIFORM

STEADY, NONUNIFORM

UNSTEADY, UNIFORM

UNSTEADY, NONUNIFORM

STOCHASTIC

INTERFACE TOPICS

AEROELASTICITY

CONTROL SYSTEMS

SHUTDOWN SYSTEMS

INTERFERENCE :

WAKE MODEL DEVELOPMENT

COMPONFNT INTERFEPENCE DEVELOPMFNT 


\section{FIGURE 2. WIND TURBINE ROTOR GENERIC APPROACHES}

ATTRTBUTES

CHARACTERISTICS

CHIEF UNKNOWNS

KNOWN LIMITS

\begin{tabular}{|c|c|c|c|c|}
\hline STALL CONTROL & $\begin{array}{l}\text { FIXED PITCH, CONSTANT RPM } \\
\text { ROTOR MUST SHED LOAD } \\
\text { AIRFOILS AT HIGH C } \mathrm{C}^{\prime} \text { 's } \\
\text { LOW ROTOR RPM }\end{array}$ & $\begin{array}{l}\text { BEEFY BLADE } \\
\text { NEAR STALL MUCH OF THE TIME } \\
\text { PROBABLY TRAILING EDGE STALL } \\
\text { HIGH SOLIDITY }\end{array}$ & $\begin{array}{l}\text { UNSTEADY AIRLOADS } \\
\text { STALL PREDICTION } \\
\text { ROUGHNESS EFFECTS }\end{array}$ & $\begin{array}{l}\text { STALL FLUTTER } \\
\text { BFTZ LIMIT }\end{array}$ \\
\hline PITCH CONTROL & $\begin{array}{l}\text { VARTABLE PITCH } \\
\text { CONSTANT RPM } \\
\text { PITCHING SHEDS LOAD } \\
\text { ROTOR AT LOW C'S } \\
\text { HIGH RPM FOR CENTRIF. RELIEF }\end{array}$ & $\begin{array}{l}\text { LOW! SOLIDITY POSSIBLE } \\
\text { BEIOW STALL, BUT STILL } \\
\text { GUST SENSITIVE } \\
\text { VARIOUS AIRFOILS ARE POSSIBLE }\end{array}$ & $\begin{array}{l}\text { UNSTEADY AIRLOADS } \\
\text { TRANSIENT AIRLOADS DUE } \\
\text { TO PITCH RATE, ETC. } \\
\text { ROUGHNESS EFFECTS }\end{array}$ & BETZ LIMIT \\
\hline VARIABLE SPEED (HAWT) & $\begin{array}{l}\text { VARIABLE ROTOR RPM } \\
\text { MUST CONTROL DRIVEN LOAD } \\
\text { NEEDS LOAD CONTROL ABOVE RATED }\end{array}$ & $\begin{array}{l}\text { ROTOR INERTIA DETERMINES } \\
\text { GUST RESPONSE } \\
\text { LOW SOLIDITY POSSIBLF } \\
\text { AERO \& INERTIAL LOADS BALANCE }\end{array}$ & $\begin{array}{l}\text { RANGE OF EFFECTIVE LOAD } \\
\text { CONTROL BEFORE RATED } \\
\text { UNSTEADY AIRLOADS } \\
\text { EFFECT OF ROUGHNESS }\end{array}$ & BETZ LTMIT \\
\hline DARRIEUS (VAWT) & $\begin{array}{l}\text { TROPOSKEIN SHAPE ROTOR BLADES } \\
\text { FIXED PITCH } \\
\text { CONSTANT RPM } \\
\text { ROTOR AIRFOIL SHEDS LOAD }\end{array}$ & $\begin{array}{l}\text { INDEPENDENT OF WIND DIRECTION } \\
\text { ALL OPERATION IN UNSTEADY } \\
\text { FLOW }\end{array}$ & UNSTEADY AERODYNAMICS & $\begin{array}{l}\text { BETZ LIMIT } \\
\text { MAY NOT APPLY }\end{array}$ \\
\hline STRAIGHT BLADE (VAWT) & $\begin{array}{l}\text { PITCH CONTROL DEGREE OF FREEDOM } \\
\text { STRAIGHT ROTOR BLADES } \\
\text { VARIABLE SPEED POSSIBLE }\end{array}$ & $\begin{array}{l}\text { VERY SENSITIVE TO PITCH } \\
\text { ANGLE CHANGES } \\
\text { CAN EXPLOIT DYNAMTC STALL } \\
\text { IF PITCH DOF, NO YAW INDEP. }\end{array}$ & UNSTEADY AERODYNAMICS & $\begin{array}{l}\text { BETZ LIMIT } \\
\text { MAY NOT APPLY }\end{array}$ \\
\hline HIGH TIP SPEED (HAWT) & $\begin{array}{l}\text { LOW SOLIDITY } \\
\text { HIGH TIP SPEED } \\
\text { LOW ROTOR C 'L'S }\end{array}$ & $\begin{array}{l}\text { HIGH TIP NOISE } \\
\text { THIN AIRFOILS, BETTER Re } \\
\text { LOW DRAG AIRFOIL POSSIBLE } \\
\text { LOWER GUST SENSITIVITY }\end{array}$ & $\begin{array}{l}\text { UNSTEADY AIRLOADS } \\
\text { EFFECT OF ROUGHNESS }\end{array}$ & $\begin{array}{l}\text { TIP MACH NO. } \\
\text { BETZ LIMIT }\end{array}$ \\
\hline FREE YAW (HAWT) & $\begin{array}{l}\text { ROTOR DYNAMIC STABILITY } \\
\text { NECESSARY } \\
\text { NO MECHANICAL OR AERODYNAMIC } \\
\text { YAW DEVICES }\end{array}$ & YAW DAMPER. ONLY & $\begin{array}{l}\text { YAW STABILITY DERIVATIVE } \\
\text { EFFECT OF STALL } \\
\text { UNSTEADY AIRLOADS }\end{array}$ & BETZ LIMIT \\
\hline
\end{tabular}


limitations, voids in knowledge, and R\&D needs. For example, a stallcontrolled rotor does not employ pitch changes to limit loading, but instead relies on airfoil stall. At some point in the engineering development of any wind turbine system a design choice is made, with all its associated tradeoffs. The currently most promising generic approaches were identified by the Panel and are described in Appendix 3. None is now clearly superior; therefore, all should be considered in defining the research goals of a successful R\&D program. Figure 2 lists the generic approaches, their general aerodynamic characteristics, chief unknowns, and present known limitations.

\section{HIGHEST PRIORITY AERODYNAMIC STUDY AREAS}

The Panel established numerical priorities for each aerodynamic topic within each generic approach. (see Figure 3). The most promising study topics could then be identified and given a priority rating.

The highest priority R\&D needs are those common to all design approaches, that cut across design and configuration boundaries, and will enhance these approaches and others yet to be identified. 
3 Highest Priority

2 High Priority

1 Low Priority

0 Does Not Apply

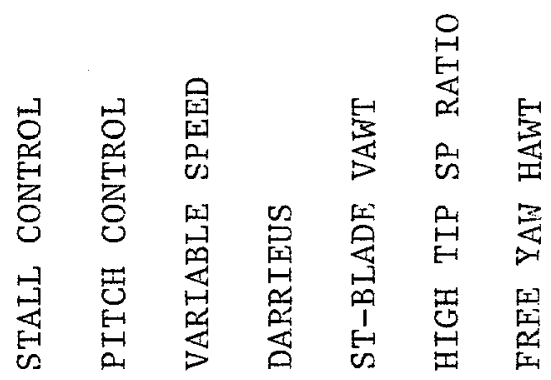

STEADY AERODYNAMTCS

1. AIRFOIL STUDIES:

2-D

3-D

EFFECT OF ROUGHNESS

AIRFOIL DEVELOPMENT

2. WAKE ANALYSIS

3. MECHANISMS :

HIGH LIFT DEVICES

SEPARATION CONTROL DEVICES

4. TESTING METHODOLOGY

UNSTEADY AERODYNAMICS

1. DYNAMIC STALL:

2-D HYSTERESIS

3-D HYSTERESIS

$$
\begin{array}{lllllll}
0 & 1 & 1 & 0 & 0 & 1 & 1 \\
1 & 2 & 2 & 0 & 0 & 1 & 2 \\
1 & 1 & 1 & 0 & 0 & 2 & 1 \\
1 & 1 & 1 & 0 & 0 & 1 & 1 \\
1 & 1 & 1 & 0 & 0 & 1 & 2 \\
& & & & & & \\
1 & 1 & 1 & 0 & 0 & 0 & 1 \\
1 & 1 & 1 & 0 & 0 & 0 & 1 \\
1 & 1 & 1 & 0 & 0 & 1 & 1
\end{array}
$$

2. AIRFOIL DEVELOPMENT STUDIES:

DELAYED STALL

SOFT STALL

REPEATABLE STALL

INSENSITIVE STALL

EFFECT OF ROUGHNESS

3. AIRFOIL DEVICES

4. TESTING METHODOLOGY:

WIND TUNNEL TESTING

TOTAL SYSTEM (FIELD) TESTING

5. PREDICTION OF PERFORMANCE

6. ROTOR STABILITY

$\begin{array}{lllllll}3 & 3 & 3 & 3 & 3 & 2 & 3 \\ 3 & 3 & 3 & 3 & 3 & 2 & 3\end{array}$

INFLOW MODELS:

1. STEADY, UNIFORM

2. STEADV, NONUNIFORM

3. UNSTEADY, UNIFORM

4. UNSTEADY, NONUNIFORM

5. STOCHASTIC

$\begin{array}{lllllll}2 & 2 & 2 & 3 & 3 & 1 & 2 \\ 2 & 2 & 2 & 3 & 3 & 2 & 2 \\ 3 & 3 & 3 & 3 & 3 & 2 & 3 \\ 3 & 3 & 3 & 3 & 3 & 2 & 3 \\ 3 & 3 & 3 & 3 & 3 & 2 & 3 \\ 2 & 2 & 2 & 3 & 3 & 1 & 2\end{array}$

INTERFACE TOPICS:

1. AEROELASTICITY

2. CONTROL SYSTEMS

3. SHUTDOWN SYSTEMS

4. INTERFERENCE:

WAKE MODEL DEVELOPMENT

COMPONENT INTERFERENCE DEVELOPMENT

$\begin{array}{lllllll}3 & 3 & 3 & 3 & 3 & 3 & 3 \\ 3 & 3 & 3 & 3 & 3 & 2 & 3 \\ 2 & 2 & 2 & 3 & 3 & 2 & 3 \\ 2 & 2 & 2 & 0 & 0 & 2 & 3 \\ & & & & & & \\ 1 & 1 & 1 & 3 & 3 & 1 & 1 \\ 2 & 2 & 2 & 1 & 1 & 2 & 3 \\ 3 & 3 & 3 & 1 & 1 & 3 & 3 \\ 3 & 3 & 3 & 1 & 1 & 3 & 3 \\ 2 & 2 & 2 & 1 & 1 & 2 & 2\end{array}$

FIGURE 3. WIND TURBINE RESEARCH NEEDS AND PRIORITIES 


\section{CHAPTER 3. WIND TURBINE AERODYNAMIC STUDY AREAS}

This Chapter defines and describes the aerodynamic study areas which were judged by the Panel to be relevant to wind turbines, and which were considered in detail for each generic design concept. The three main categories are:

- Airfoil and rotor behavior, i.e., steady aerodynamics and unsteady aerodynamics,

0 Inflow, and

o Interfaces.

Below, each of these is further broken down into specific subcategories, developed in order of their sophistication or difficulty. The list is a logical progression of complexity, from the simplest (or existing) theory to the complex (or not yet understood). These categories and study areas are all shown in Figure 1.

\section{AIRFOIL AND ROTOR BEHAVIOR}

The first category is the interaction between the airflow and the airfoil. All the aerodynamic manifestations are due to the integrated friction and pressure forces on this level.

A. Steady Aerodynamics. Most classical aerodynamics, and all present design tools for wind turbines, fall into this category.

1. Airfoil Studies: The following study areas are centered on the airfoil or blade element, and are the "building blocks" for all which follows.

a. Two Dimensional (2-D).

Virtually all textbook and reference data fall into this category of study. Most wind-tunnel testing and comparisons of airfoils and airfoil families have been done at the 2-D level because of its powerful mathematical generality. Strip theories, which are the current aerodynamic design tools, all depend on 2-D, steady-state airfoil data. The 2-D steady-state description is the state-of-the-art in predicting stall of a rotor blade, whether on a helicopter or wind turbine.

b. Three Dimensional (3-D). The extension to threedimensional flow allows treatment of tip effects, spanwise flow, and radial pressure gradients. In conventional aircraft studies these are called aspect ratio effects. Three-dimensional flow also includes, for rotary-wing aircraft and wind turbines, the induced velocity field in the rotor plane caused by the strong helical vortex wake shed by the blades.

c. Effect of Roughness.

This study is concerned with surface effects on airfoil behavior, which is significantly affected by the roughness caused by a number of environmental factors including dirt, bugs, rain, ice, and photochemical degradation. Figure 4 [4] shows representative types of airfoils, their steady-state stalling behavior, and the effect of roughness. For example, there is recent evidence that surface roughness does not affect the stall of certain airfoils as significantly as other commonly used airfoils [5].

\section{d. Airfoil Development.}

Designers introduce new airfoils and modify existing ones in order to meet new requirements or to improve performance. Invariably the basis of the underlying mathematical treatment is 2-D,steady-state aerodynamics. For example, current computer codes for airfoil design, e.g., the Eppler Code, tailor an 


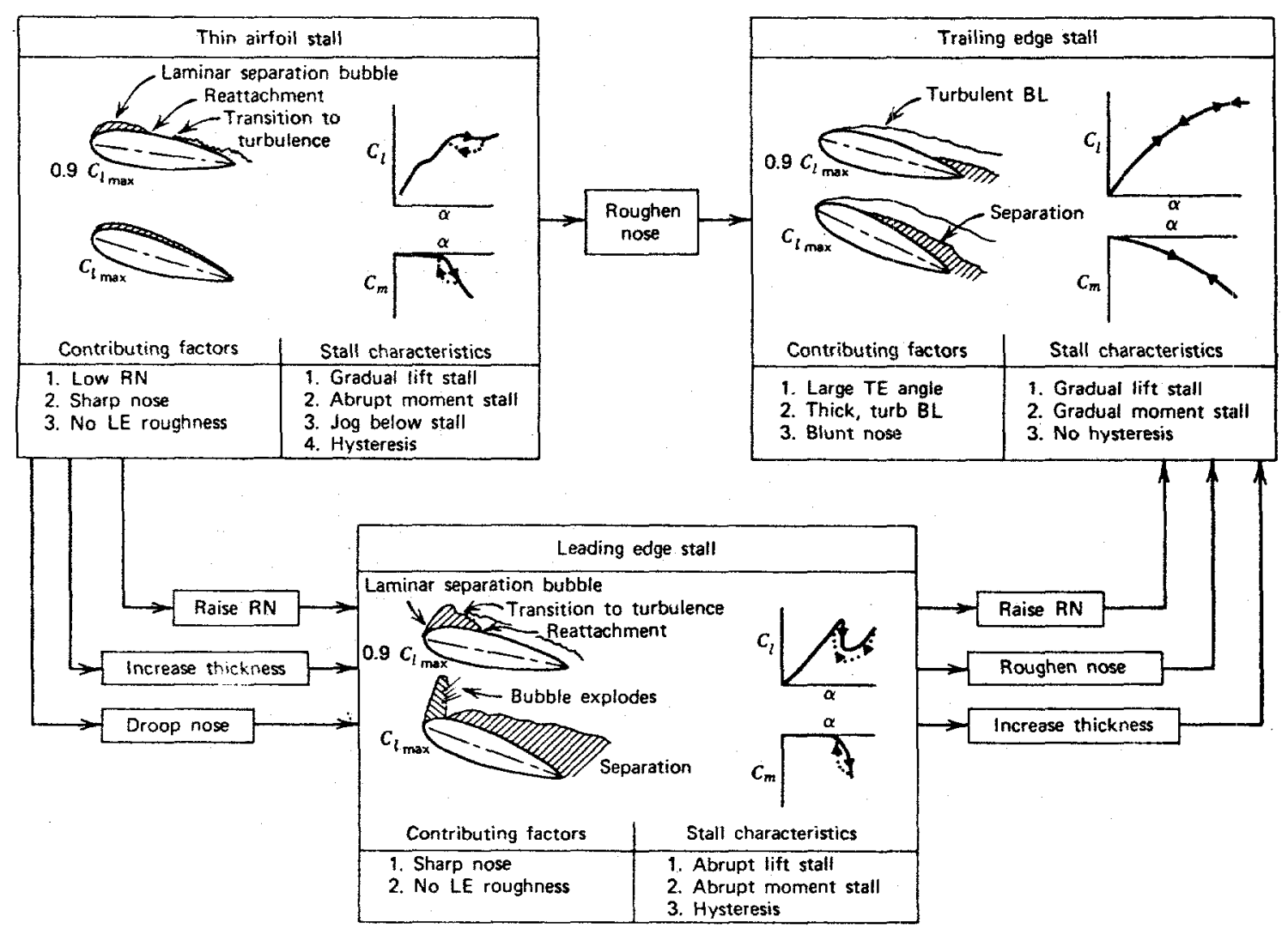


airfoil shape to a given table of desired conditions and parameters [6]. In the past, much airfoil development (e.g., the NACA 4- and 5-digit series foils) was done empirically in wind tunnels.

2. Wake Analysis: This is the study of the vortex wake of a rotor or propeller, in order to assess the induced velocity caused by this wake; see Figure 5 [7]. This induced velocity results in angle of attack changes along the blade which must be accounted for in order to predict loading. A good approximation of the vortex wake geometry and induced velocity is essential for good performance analysis.

3. Mechanisms: Mechanisms are added to otherwise clean aerodynamic devices in order to extend the operating envelope or improve performance for certain conditions. For example, fixed-wing aircraft have trailing-edge flaps which generate large lift to allow very slow landing and takeoff speeds, without stall. Devices are also used to increase performance in other areas.

a. High-Lift Devices. As
mentioned above, these are used to extend the flight envelope of wings and (sometimes) rotor blades, to allow flight in conditions beyond normal operations, which is simply cruise in the case of fixed-wing aircraft.

\section{b. Separation Control Devices}

These include vortex generators, flow energizers, attachment promoters, and any other devices which attempt to delay the stalling or separation of a given aerodynamic surface which would normally otherwise be stalled or separated; see Figure 6 [8]. Again, these are used to extend the envelope of flight, and are usually used to enhance controllability or consistency rather than directly increase lift or speed.
4. Testing Methodology: Practically all steady-state aerodynamic testing is done in wind tunnels. These wind-tunnel data are usually time series results or compilations of average data. Some flight data are used under conditions where a great deal of effort is expended to produce as close to steady state as possible.

B. Unsteady Aerodynamics. Unsteady aerodynamics is concerned with airfoil and rotor behavior under time-varying conditions. Unsteady effects have been recognized since the early days of airfoil investigation. Often an unsteady aerodynamic phenomenon, for example, a hummingbird's flight, can be described and explained qualitatively, but cannot be described mathematically. Without a sensible mathematical description, the effects, both detrimental and beneficial, cannot be assessed. The study of airfoils in unsteady conditions is important for aircraft, but is essential for wind turbines.

1. Dynamic Stall: Dynamic stall is described in Appendix 2 [9]. Its associated loss of lift and impulsive pitching moment occur as a result of many factors, including airfoil shape, starting angle of attack, rate of change of angle of attack, and the starting point of the cycle (that is, the time history of the motion). The resulting stall is usually represented as a hysteresis loop on the traditional 2-D lift and moment airfoil curves; see Figure 7. When dynamic stall occurs, it is almost always unexpected, abrupt, and inconsistent, and has significant effects on rotors and aircraft.

a. Two-Dimensional Hysteresis. The most basic level of study of dynamic stall assumes a blade of "infinite aspect ratio" 
FIGURE 5. ROTOR WAKE GEOMETRY [7]

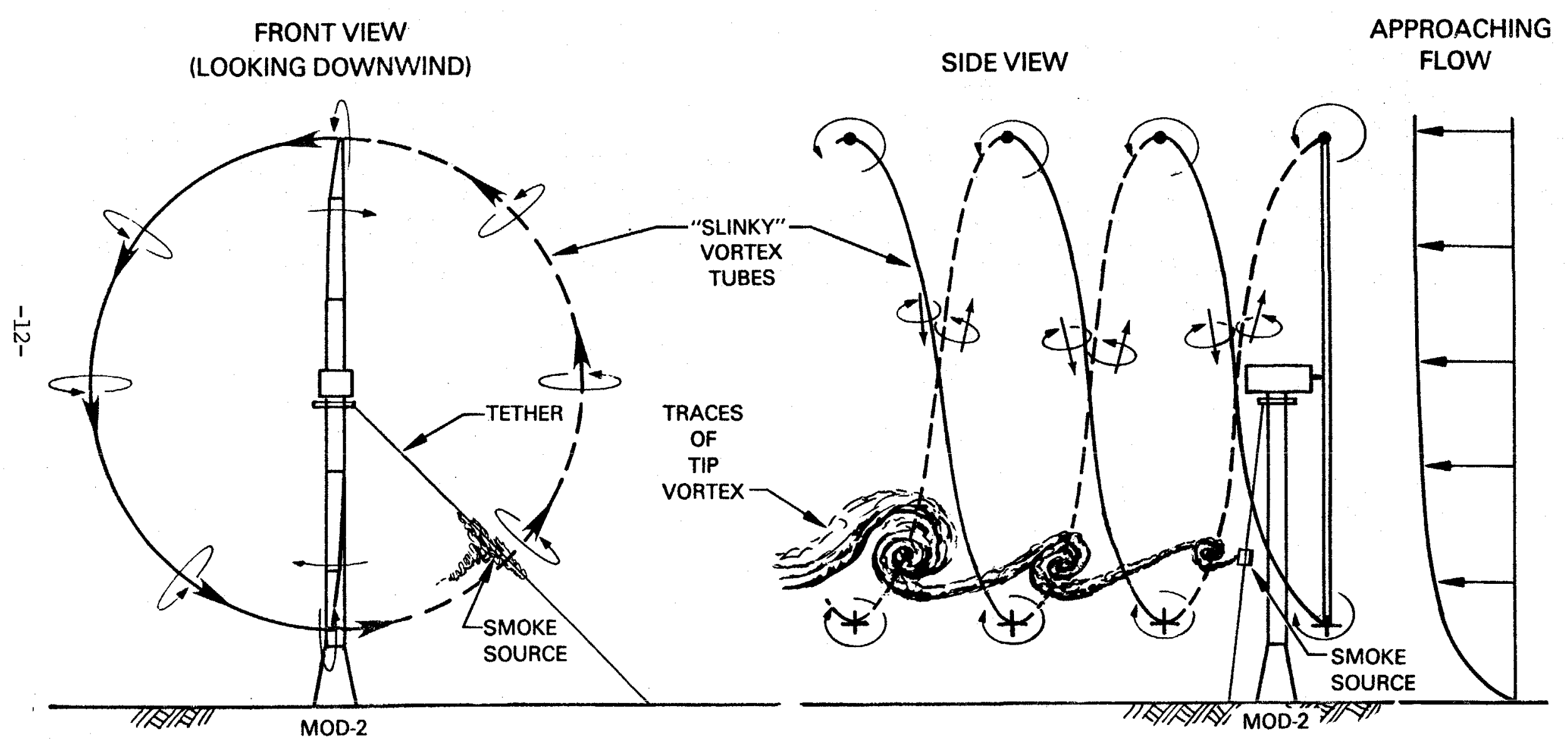




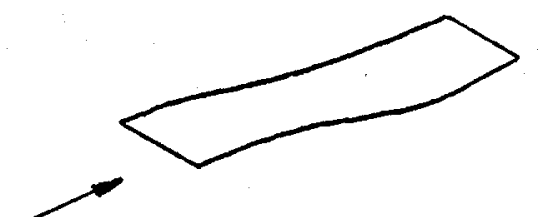

Simple Plow
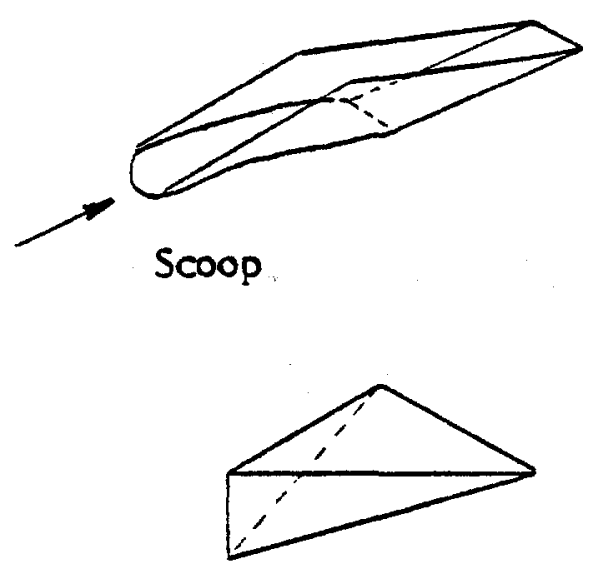

Wedge

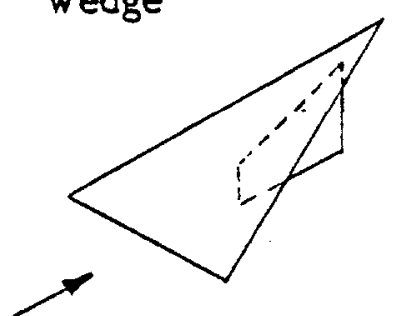

Ramp

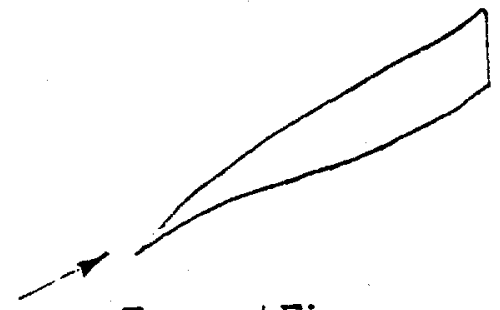

Tapered Fin

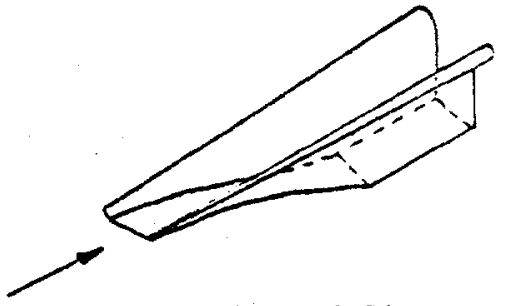

Shielded Plow
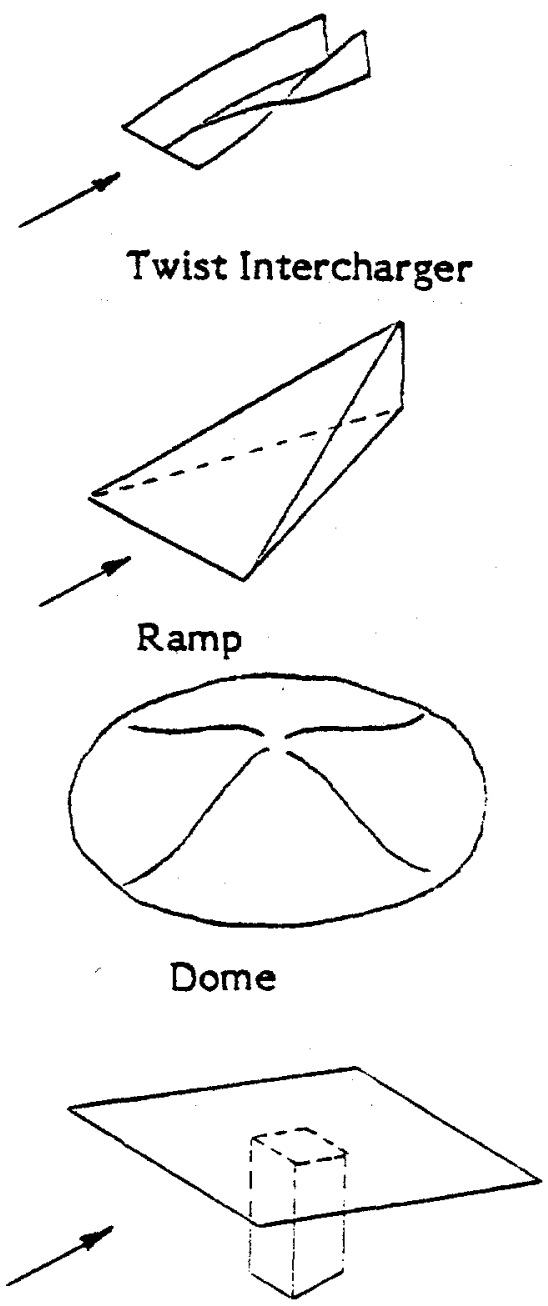

Finite Wing 
FIGURE 7. DYNAMIC STALL OF NACA 0012 AIRFOIL [9]

$$
\alpha=15^{\circ}+10^{\circ} \sin \omega t \quad k=\frac{\omega c}{2 U_{\infty}}=0.15 . \quad R e=2.5 \times 10^{6}
$$

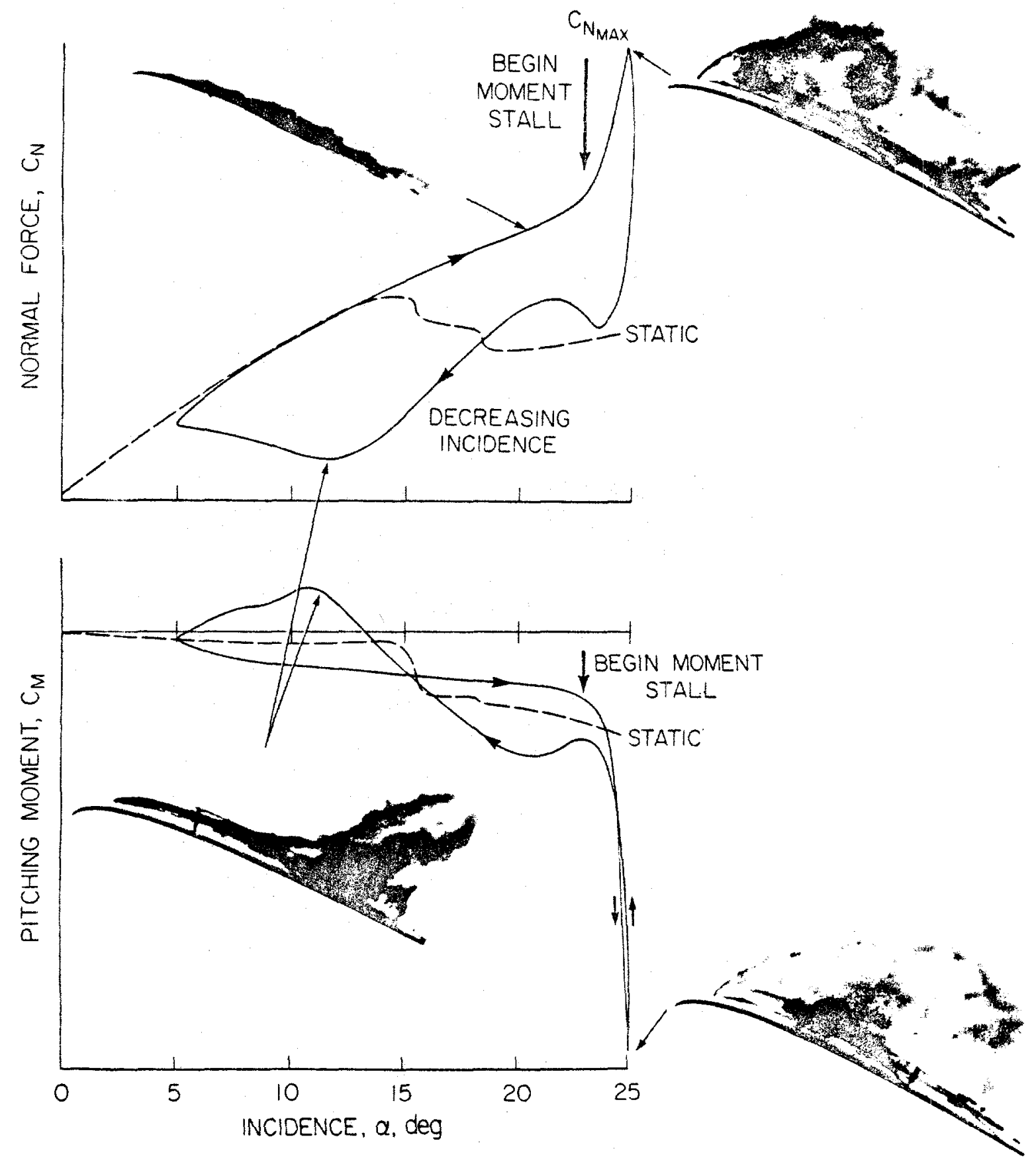


i.e., 2-D) oscillating under controlled conditions in a wind tunnel. This is an attempt to produce conditions which are identical along the test blade, or truly two-dimensional. Such studies are usually concerned with determining what set of conditions and time histories produce what hysteresis effects for a given airfoil.

\section{b. Three-Dimensional Hyste-} resis. As in steady-state work, the next step is to introduce finite span, or radial pressure gradients. Again, three-dimensional studies attempt to assess the effects of phenomena such as spanwise flow, sweepback, and tip effects on dynamic stall.

2. Airfoil Development Studies: When sufficient work is done to understand, describe and sensibly predict dynamic stall, it should be possible to design airfoils and blades which exploit dynamic stall effects. There appears to be a large amount of manipulation possible, judging from recent tests [10]. Some effects may be possible with simple airfoil geometry modifications, while others may require devices such as flaps or slats.

One of the interesting features of dynamic stall is that all airfoils seem to behave the same once the dynamic stall events have reached an irreversible point, but they behave very differently up to that point. It is also very important in wind turbine applications that the unsteady effects described below have no associated drag penalty since that would significantly reduce the output torque of the wind turbine. a. Delayed Stall. The first two categories, delayed stall and soft stall, avoid the hysteresis of the irreversible dynamic stall event. Both approaches cause airfoil lift and moment to exceed static values for a short time and then return to the static values, with no other penalty. Delayed stall is depicted on the curve in Figure 8. It has been reproduced in wind tunnel tests [10], wherein the static stall point was greatly exceeded during a dynamic airfoil pitching cycle (also shown on the curve). The associated pitching moment curve also did not contain the large impulsive moment hysteresis. If this airfoil modification is used, lift coefficients will reach very high momentary values and the lifting flow will remain intact. Essentially, the lift-curve slope stays constant, as can be seen in Figure 8. A consequence of this for rotors is that the aerodynamic damping in flapping motion is dependent on this being true.

b. Soft Stall. Soft stall, also depicted in Figure 8, is more difficult to achieve than delayed stall. Here the lifting flow is not completely lost, since the values return to the static values after the cycle just as above; the lift coefficient does not continue to rise, but levels off instead, leading to the term "soft stall." The large impulsive airloads present in delayed stall will not occur, but aerodynamic damping will be affected since the lift-curve slope has been changed. c. Repeatable Stall. One of the most difficult aspects of unsteady aerodynamics is the wide variation of response under apparently identical conditions. An example is the stall-spin event in fixed wing aircraft; even under the apparently same conditions of stall entry, one time the left wing might break, and another time the right one might. Present consensus about this is that the outcome is very sensitive to flow details such as microturbulence. Similarly, even under apparently identical wind-tunnel conditions dynamic stall hysteresis results may have a 

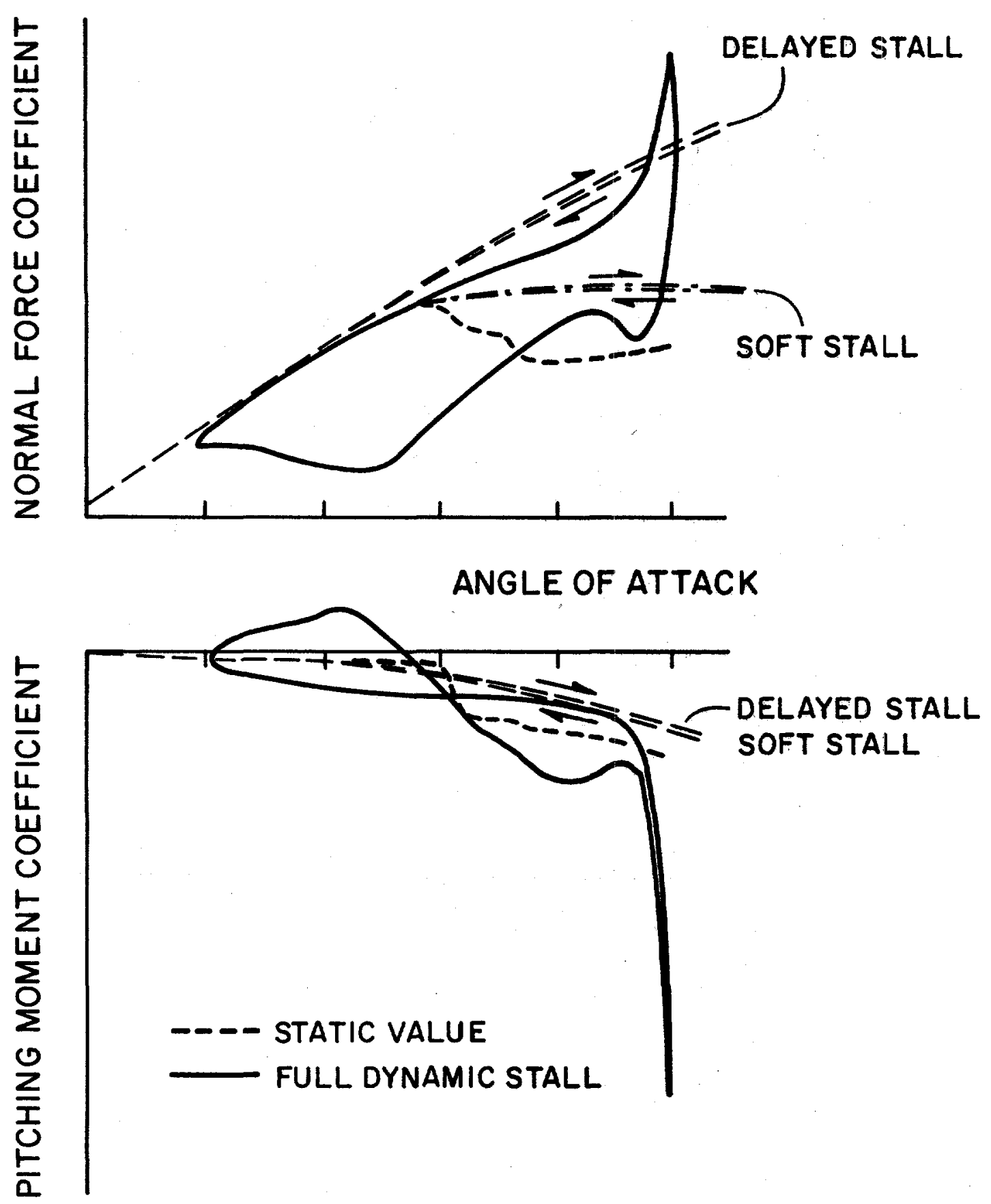
FIGURE 9. DYNAMIC STALL VARIATION

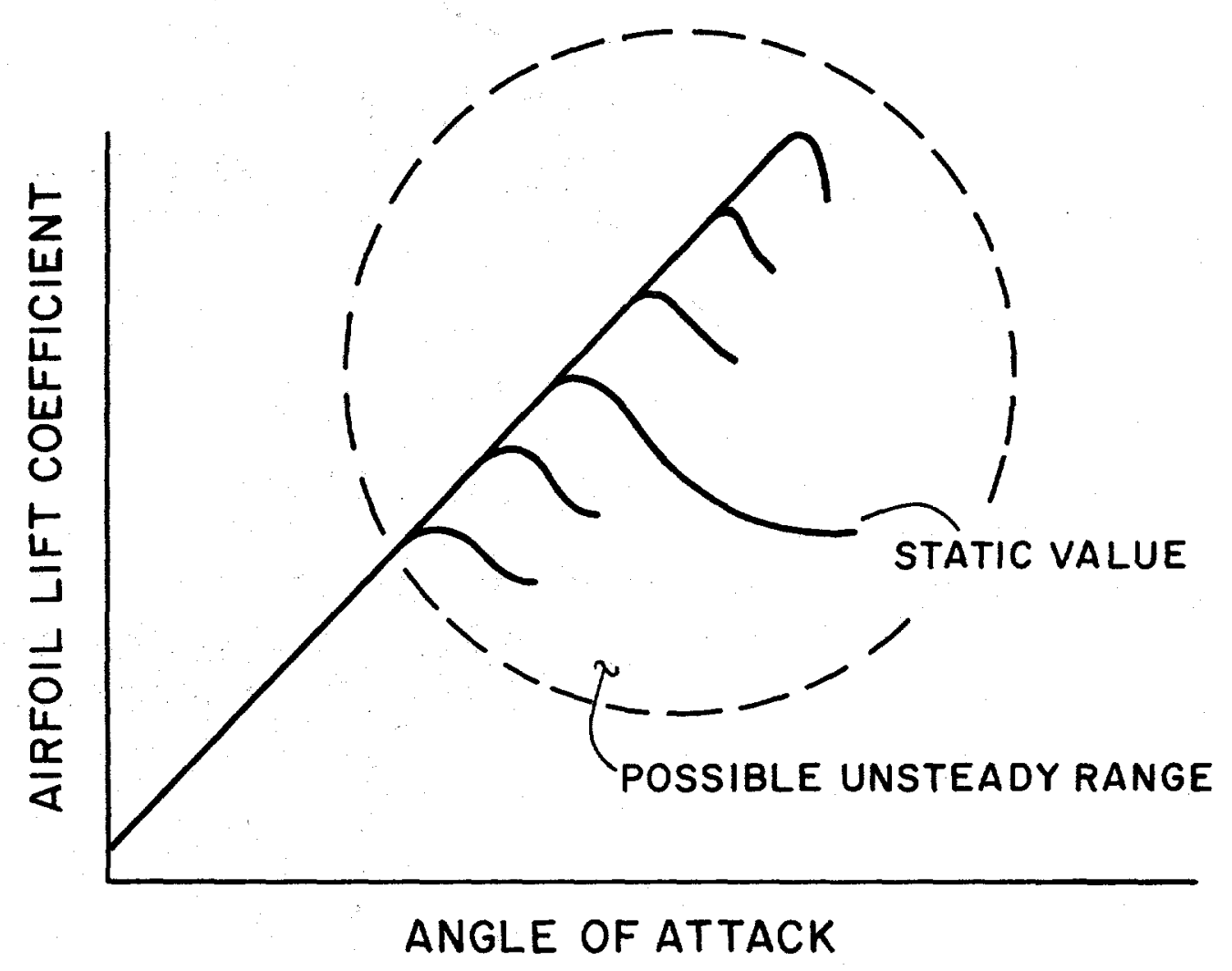


wide variation; this is depicted in Figure 9. The study of repeatable stall in unsteady conditions should reveal more about these subtle aerodynamic effects.

d. Insensitive Stall. Once the unsteady "repeatability" has been described, it is possible to design it into the performance of a rotor blade or wing. Insensitive stall means simply that the airfoil will show the same hysteresis under a wide range of conditions and time histories of motion.

e. Effect of Roughness.

Airfoil surface roughness has an important effect in the steady-state case, as depicted in Figure 10 [11]. In the unsteady case, roughness is likely to have a much more significant effect, especially in view of the subtle behavior modification which may be attempted in unsteady airfoil development.

3. Airfoil Devices: Unsteady aerodynamic devices include flaps, slats, and high lift devices. Their likely uses will be to foster delayed stall, soft stall, repeatable stall, and insensitive stall. The example given under delayed stall [10] was achieved in wind-tunnel tests with a leading edge slat modification to the airfoil.

4. Testing Methodology: All the aircraft and wind turbines operating in the unsteady environment are potential testbeds for unsteady aerodynamics. In fact, aggravating fluctuating airloads caused by dynamic stall are usually discovered first in flight testing (e.g., retreating blade stall on helicopters in forward flight). Systematic study usually then follows. To achieve understanding, controlled experimentation is required, and variation of a single parameter is the objective. This is frequently difficult in wind tunnels and impossible in system testing in the field. a. Wind Tunnel Testing.

Wind tunnels are being used increasingly in the study of unsteady aerodynamics. A very clean (low turbulence) tunnel can be used for 2-D studies of dynamic stall hysteresis, for example. Other tunnels use 3-D models of terrain and buildings and simulate unsteady atmospheric flow [12]. Wind-tunnel studies of the airfoil are done under 2-D circumstances, and studies of blades and rotors in 3-D circumstances. This field is called wind engineering, and any advances in exploiting unsteady aerodynamics will probably be seen in these tests before they will be described mathematically.

$$
\text { b. Total System (Field) }
$$

Testing. There must eventually exist the capability to do complete system testing, in the field, for unsteady aerodynamics. Existing systems will be useful if adequate and effective instrumentation and conditions can be maintained.

5. Prediction of Performance: It is clear that the logical progression of aerodynamics work is to study and develop the airfoil (1), then the rotor (2), and finally the performance prediction code (3). In steady flow, rotor performance is largely determined by the vortex wake, and to date a premium has been thus placed on the study of the fixed wake. In unsteady flow, the wake strength and geometry vary, making the problem more complex.

However, describing the wake effect on the rotor is not a large step, since the analysis methods are well in hand to describe varying, nonuniform inflow effects on the angle of attack distribution of the blade. Performance includes both productivity and reliability. A three-dimensionally varying wake geometry will place vorticity near enough to the blade that impulsive 

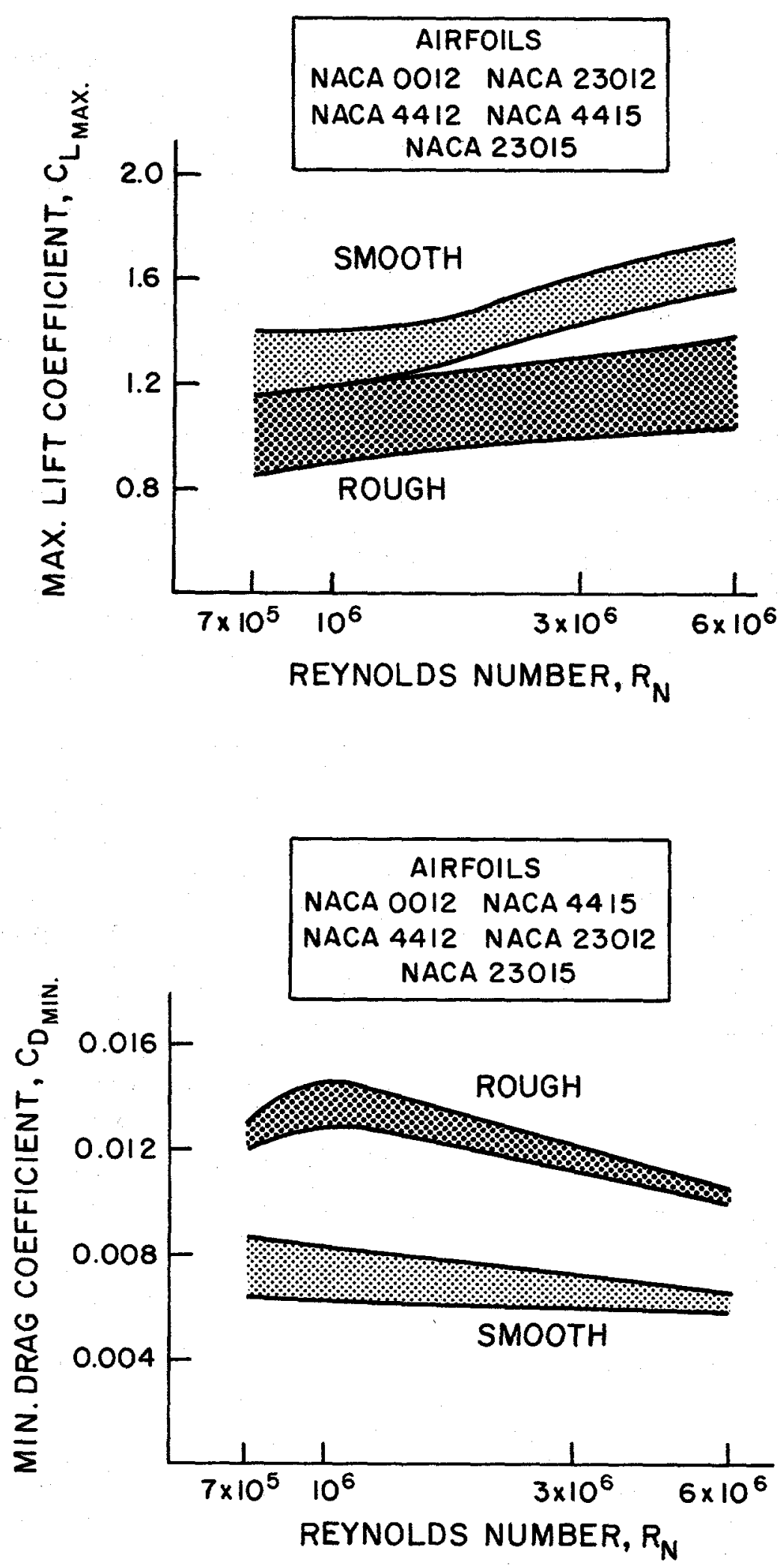
airloads will result. This unsteady effect, also seen in steady-state tests where the wake is fixed, decreases the fatigue life of the blades.

6. Rotor Stability: Stability of aircraft is complex, involving six degrees of freedom even for an assumed rigid flight vehicle; by contrast, wind turbines have only one equivalent degree of freedom, yaw. Using momentum/blade element theory (e.g., the PROP code) [13], it can be shown theoretically that in steady state, no aerodynamic yawing moment is produced by a wind turbine rotor [14]. A static yawing moment will be produced only in the presence of nonuniform induced velocity and blade motion. In unsteady conditions, yaw stability is more complex. Again, the unsteady geometry and strength of the wake are of crucial importance.

\section{INFLOW MODELS}

The second major aerodynamic topic is the inflow to the rotor, which causes the unsteady effects described above. An adequate mathematical description of the inflow will allow a variation in angle of attack of the rotor. This factor dominates the unsteady response. The inflow consists of:

o wind speed and direction fluctuations,

- terrain-induced fluctuations, and

o interference-induced fluctuations,

all of which are unsteady.

Each component can be represented by a non-stationary, 3-dimensional vector quantity, which is non-stationary. This means that it is not simply characterized by an average value and a complete set of moments of fluctuations about that mean; e.g., the average wind speed depends on the integration time and does not reach a constant even for large time [15].

The specification of these components in a form suitable for inflow calculations is far beyond present capability. One simplifying assumption is to specify only the horizontal component of inflow (wind), which is primarily responsible for angle of attack variation; this can be done via linear superposition. This makes the problem sensible and tractable for the present. A new field of study must be developed to go further; the Panel calls this aerometeorology, and discusses it in Chapter 6 of this Report.

An example of how unsteady fluctuations in the atmosphere are presently studied is given in Figure 11 for the simple case of a vertical wind gust on an aircraft in cruise. Isotropic turbulence, and a Von Karman power spectral density, are assumed. A simple cosine gust profile with a certain longitudinal wavelength is extracted from the model, and its effect on the aircraft is estimated in the following way: the atmosphere, exclusive of the gust, is assumed to be steady state for a short time. The equation of motion of the aircraft, which in general involves three space variables $(x, y, z)$ and time, is simplified by assuming simple time-dependent rectilinear flight. An effective pitching rate is calculated from the gust profile, and the resulting angle of attack change is calculated from that. Finally, the airload is calculated from the transfer function of the "point model" of the rigid aircraft dynamics.

For wind turbines, the description must be able to go far beyond this. The inflow is inherently nonuniform and unsteady. A logical 
FIGURE 11. AIRCRAFT GUST MODEL
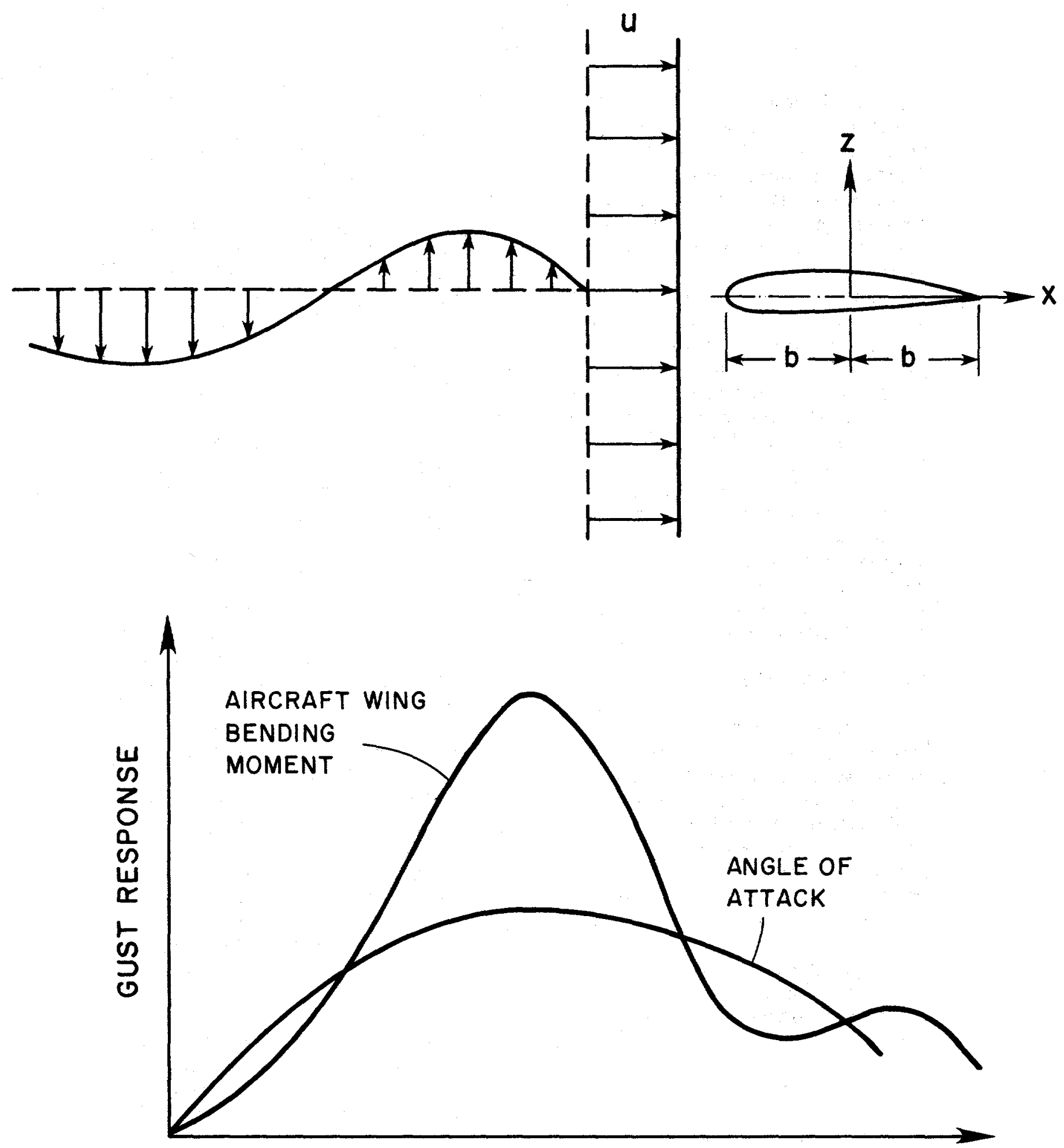

DISTANCE TRAVELED THROUGH GUST 
way to proceed is to build a hierarchy of descriptors of these components, of increasing complexity and realism, leading eventually to an ability to describe both spatial and temporal variations of the inflow. In the following descriptions of such a hierarchy, steady and unsteady refer to the time variation of the inflow, and uniform and nonuniform to the spatial variation.

A. Steady, Uniform. Steady, uniform inflow is depicted in Figure 12. This is the classical wind-tunnel flow, and admits no variation in either time or space.

B. Steady, Nonuniform. Steady, nonuniform inflow, shown in Figure 13 , has an inflow "front" which travels along with the horizontal flow. Linear wind shear might be the first term in a series representation of such a field, and a parabolic profile might be another. Any mathematical function with parametric frequency or wavelength might be used in such a series representation. Practically all present inflow modeling is at this level of description.

C. Unsteady, Uniform. Unsteady, uniform inflow is simply defined at present within the community as a "rotor gust front". The profile is uniform (constant), but the value changes with time. Hence the gust has "shape" and duration, as shown in Figure 12, and envelops the rotor uniformly. However, a given atmospheric turbulence eddy may appear nonuniform to a large diameter rotor, and uniform to a small diameter one (see Figure 14); this illustrates the need for an adequate (i.e., wavelength) description of spatial variation in the inflow.

D. Unsteady, Nonuniform. The actual inflow is both unsteady and nonuniform, as depicted in Figure 15. To first approximation, the inflow front may perhaps be taken to have 2-D shape similarity or symmetry; in general, variations will affect the front shape as well as magnitude.

E. Stochastic. Up to this point, the discussion has concerned deterministic descriptions of inflow. If a more general stochastic representation is successfully developed, the powerful tools of frequency analysis and statistical methods can be used (Figure 16). Stochastic methods are the most effective way of studying these. Most turbulence work to date in the atmospheric physics community has been accomplished in the frequency/wave number domain with statistical methods.

\section{INTERFACE TOPICS}

The third and last category of aerodynamic study concerns topics in which the aerodynamics plays a major or contributing role through the unsteady airloading, but which depend also on other disciplines.

A. Aeroelasticity. Here the major interface is with structures, which introduce both inertial and elastic forces which may lead to instabilities. Mass and motion are introduced, and depend on aerodynamic forces. The resulting feedback is also important to aerodynamic performance calculations, since blade motions affect angle of attack distributions and time variations.

B. Control Systems. Control systems are used to extend the operational envelope by decreasing loads, and to capture more energy by improving efficiency. The four control regions of wind turbines are discussed in Appendix 2 and shown in Figure 17; they are (1) 
FIGURE 12. WIND INFLOW: UNIFORM FRONT, STEADY AND UNSTEADY

\section{STEADY}
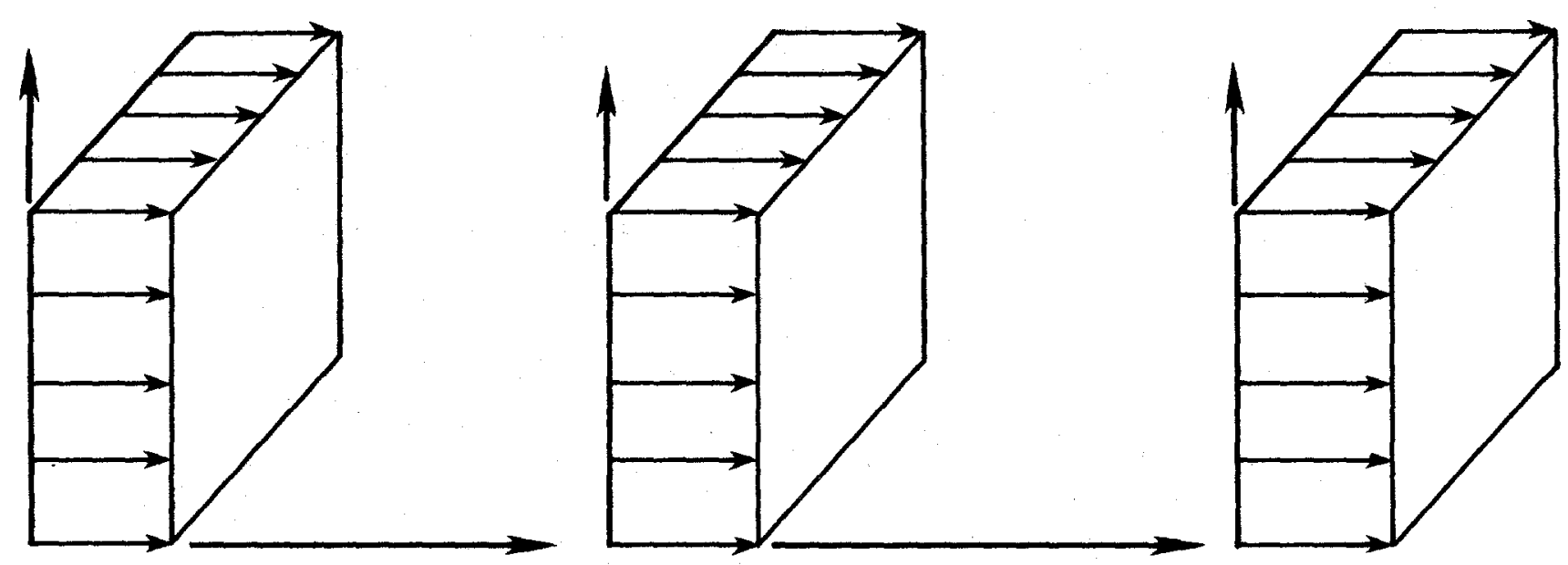

UNSTEADY
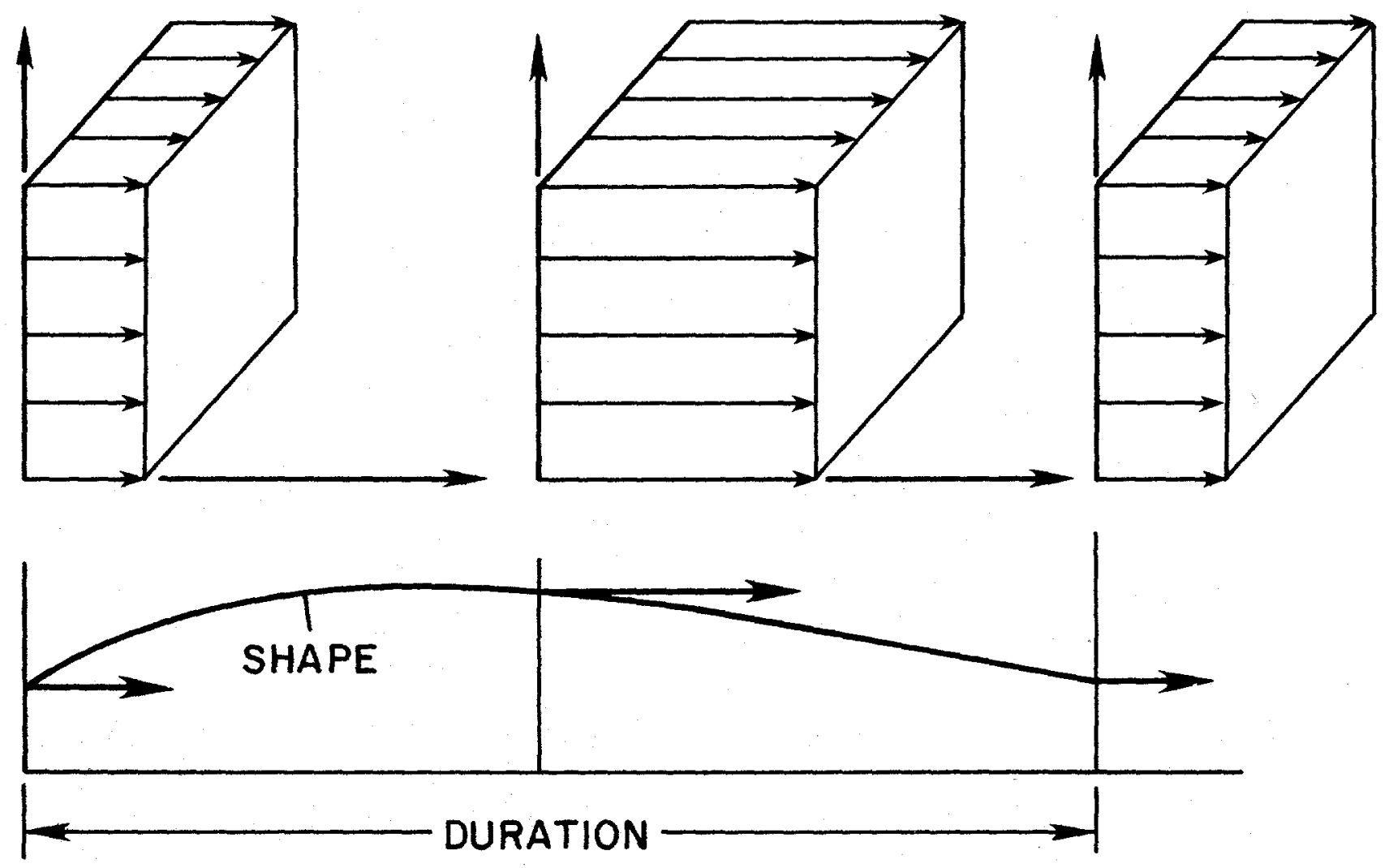
FIGURE 13. WIND INFLOW: NONUNIFORM FRONT, STEADY

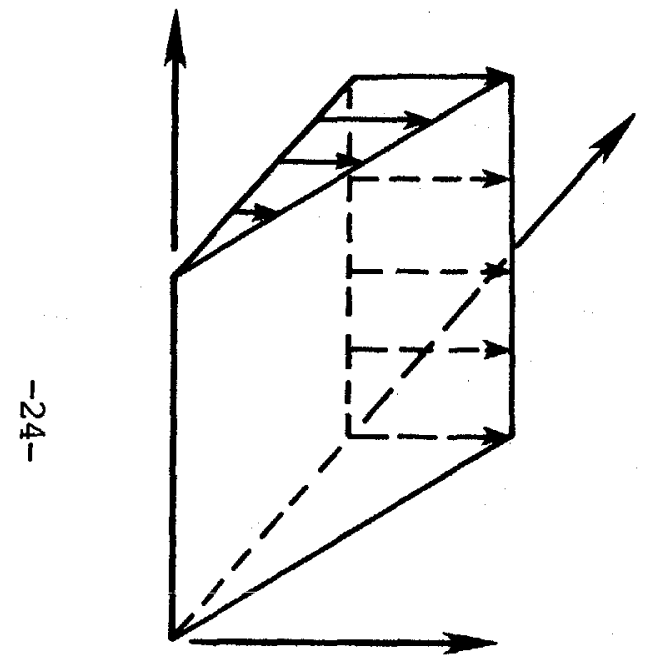

LINEAR

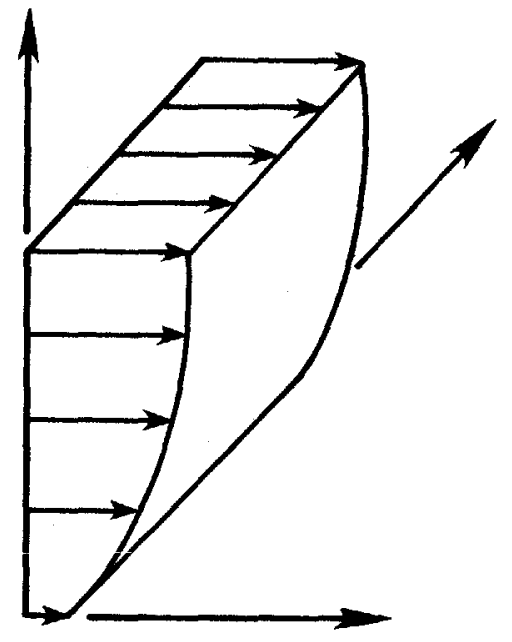

PARABOLIC

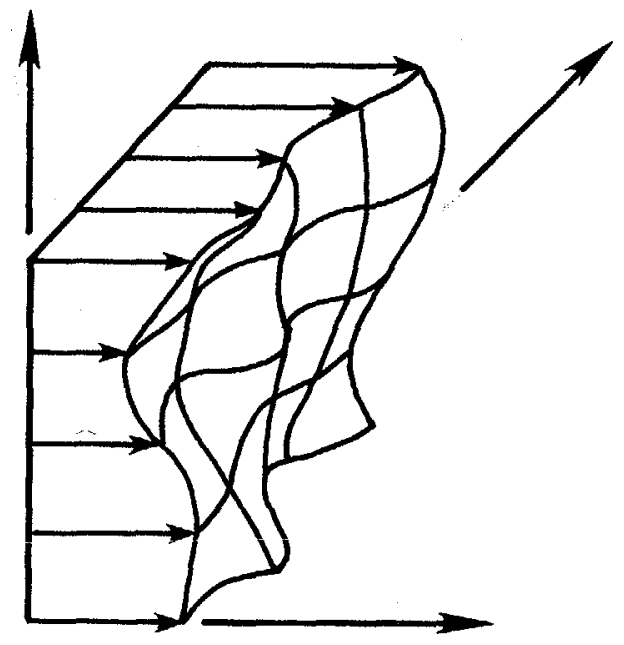

COMPLEX 


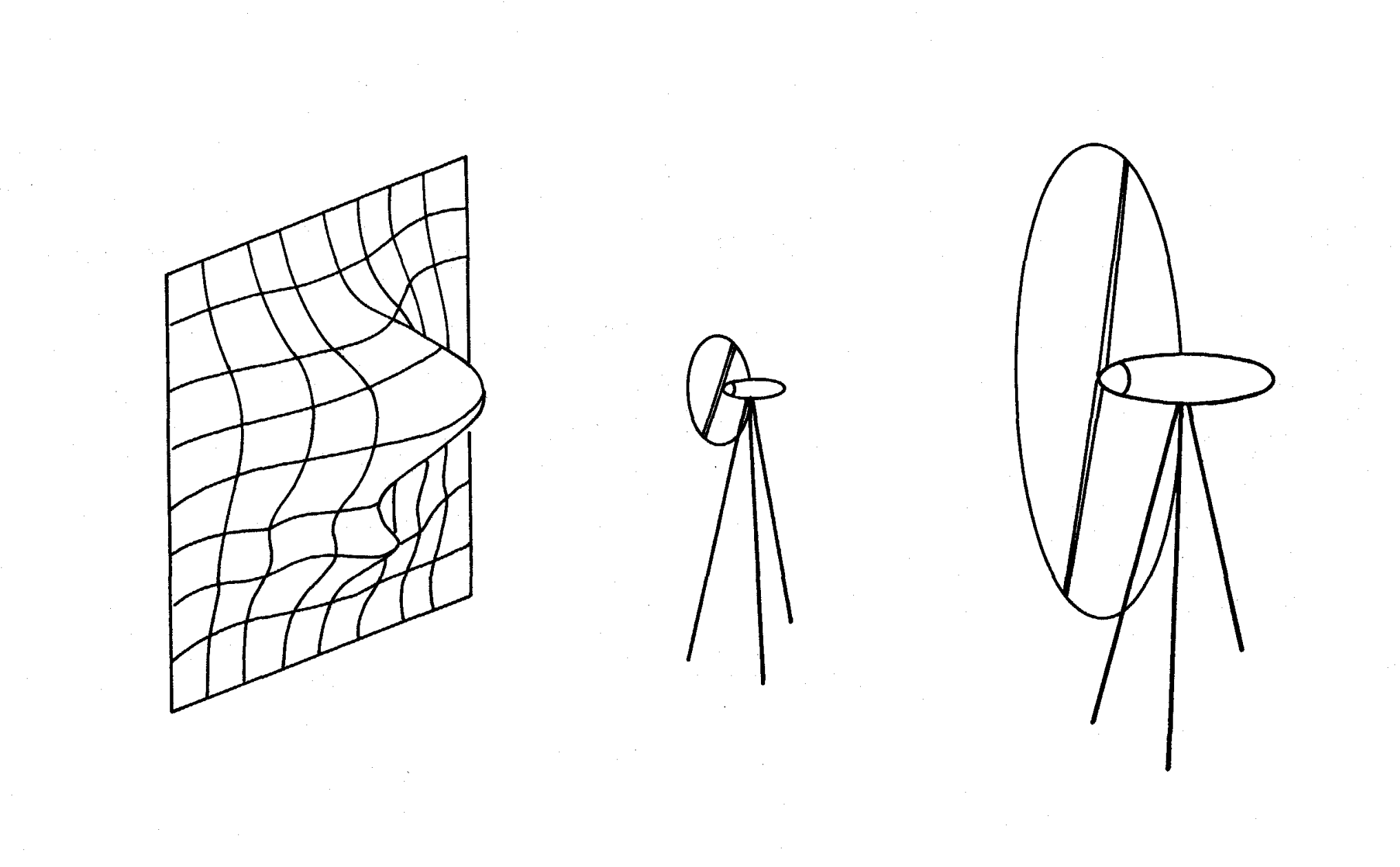



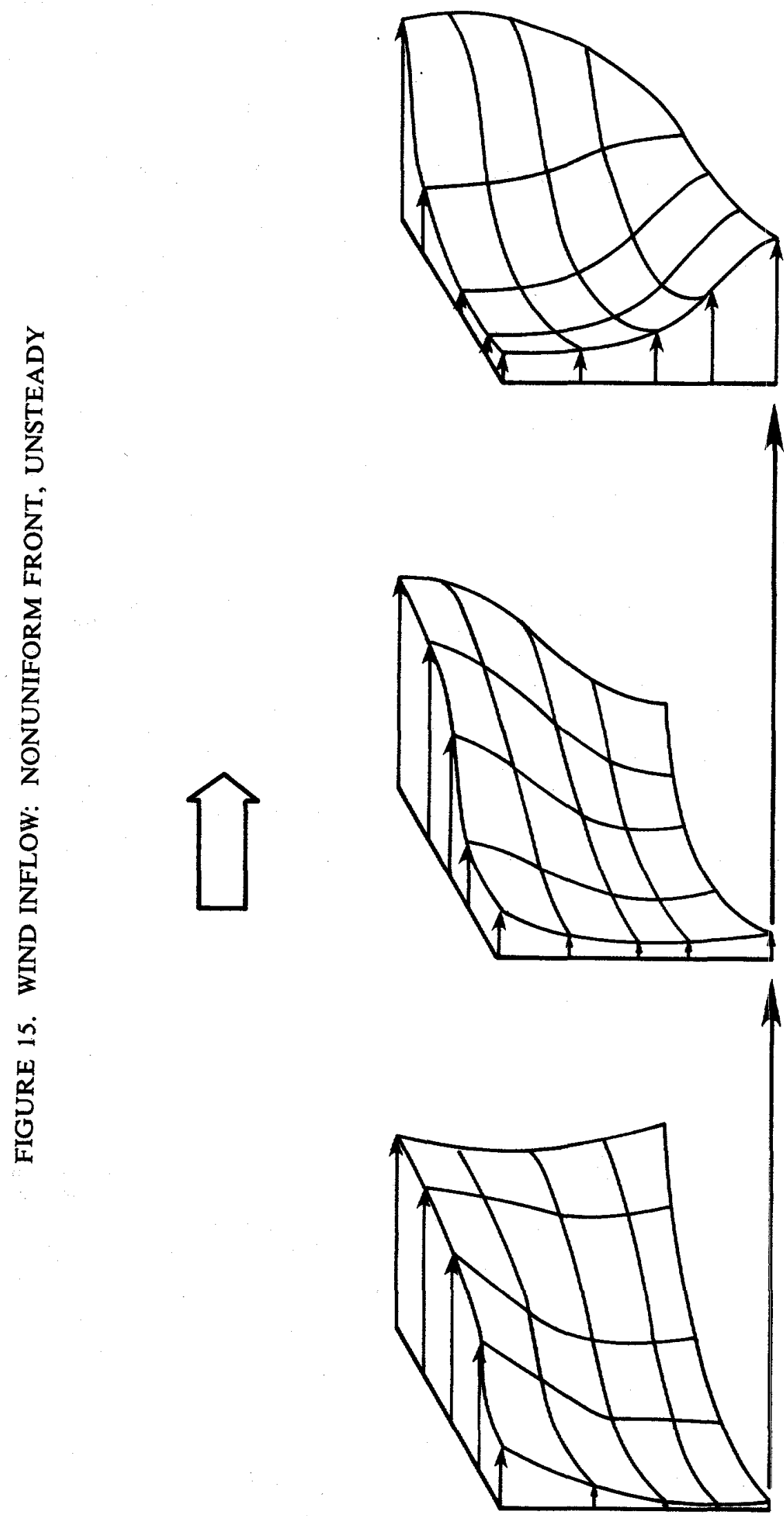
FIGURE 15. WIND INFLOW: NONUNIFORM FRONT, UNSTEADY (CONT'D)
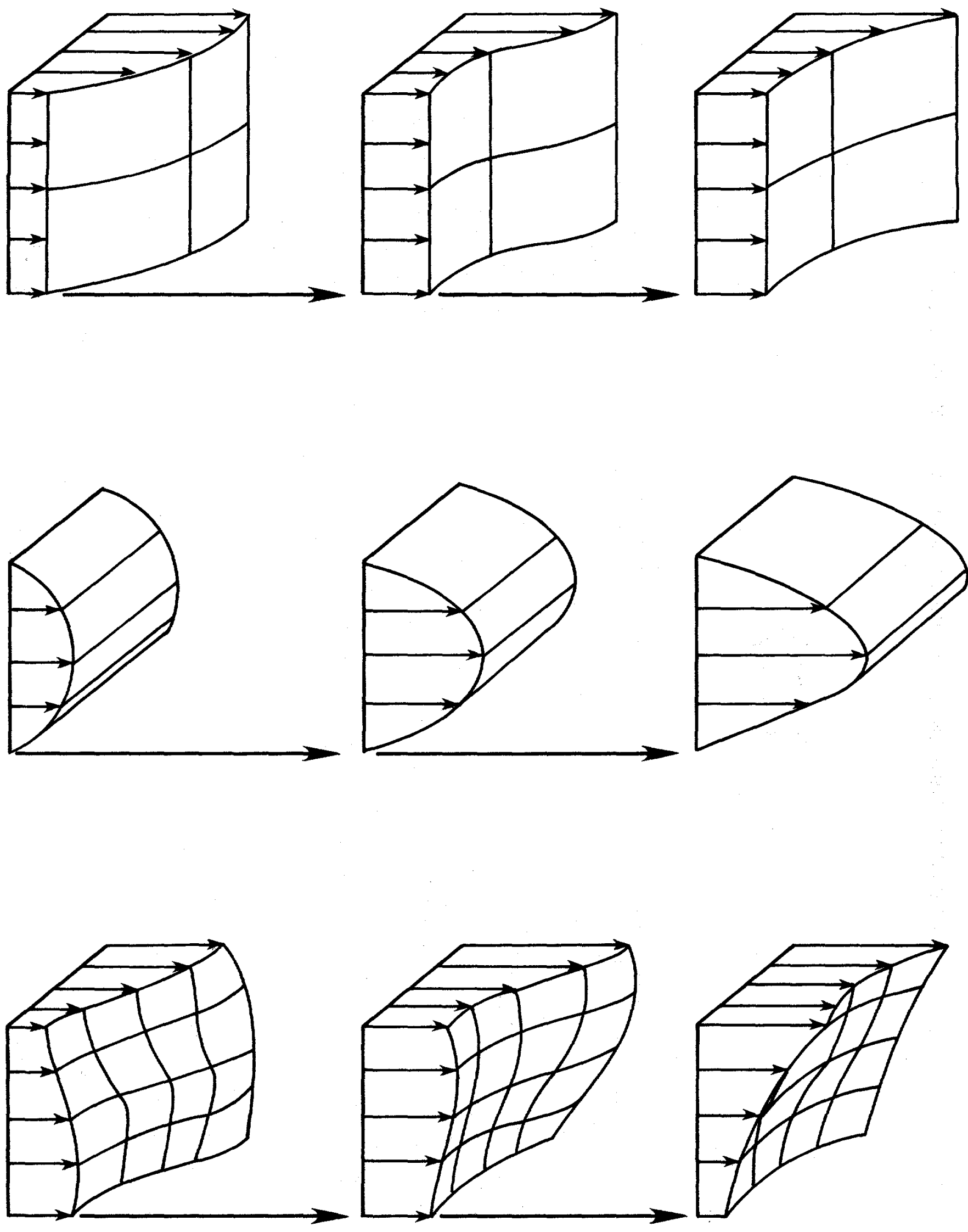
FIGURE 16. STOCHASTIC WIND INFLOW
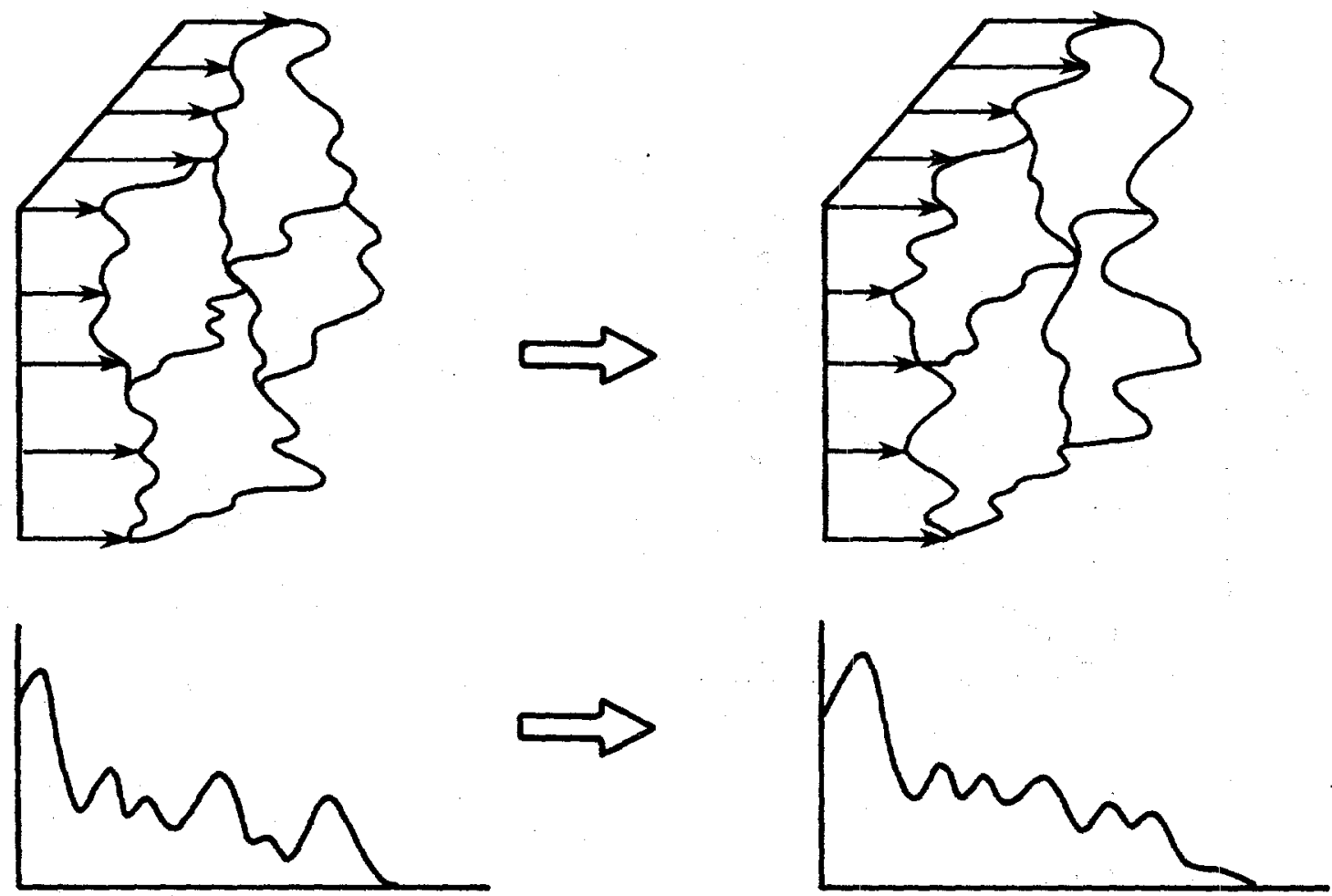

POWER SPECTRAL DENSITY
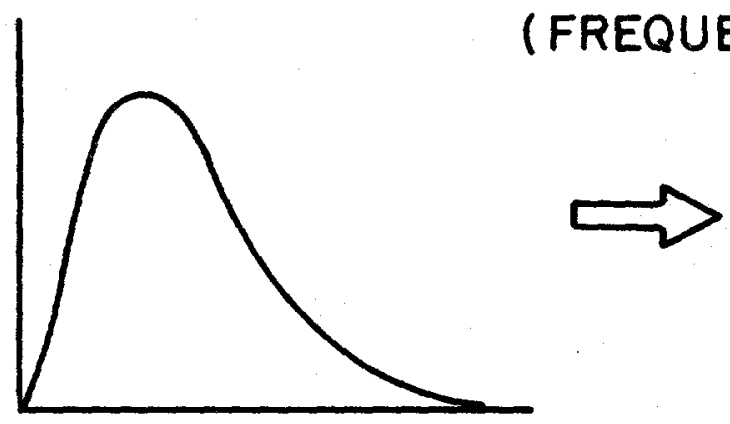

PROBABILITY DENSITY

(AMPLITUDE)

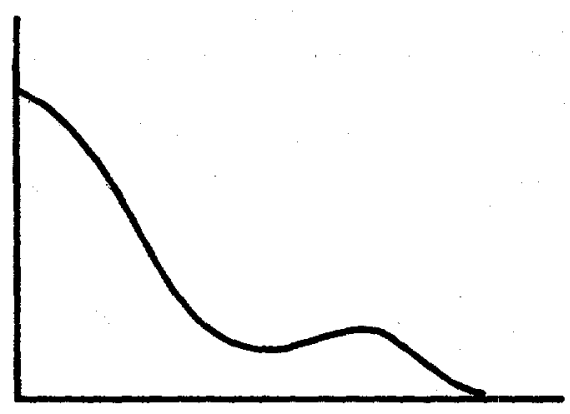

AUTOCORRELATION

( TIME) 
FIGURE 17. WIND TURBINE GENERAL CONTROL REGIONS

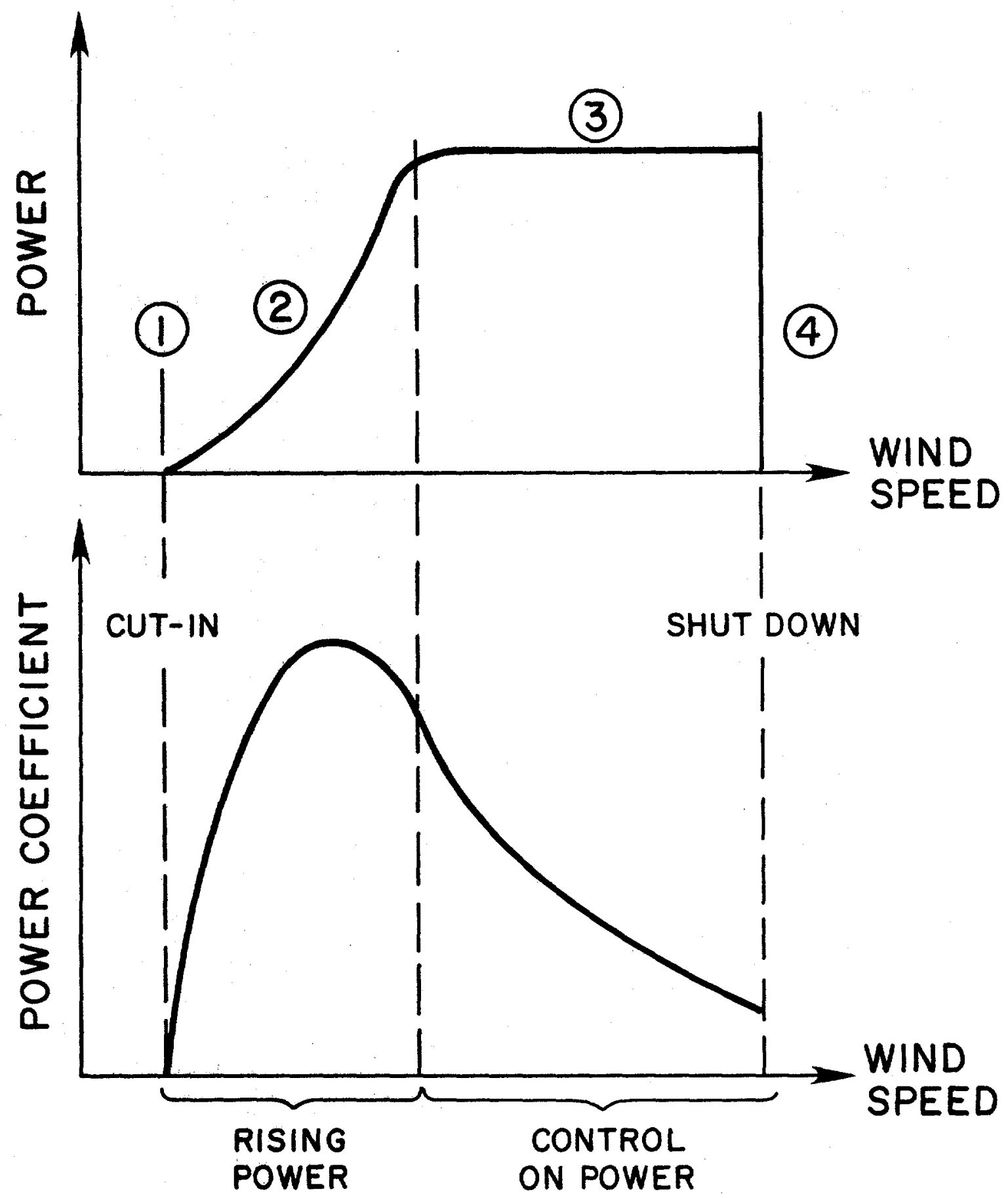


cut-in, (2) rising power, (3) load limiting, and (4) shutdown. Load limiting and power limiting are not necessarily the same; load limiting may occur first, in which case the control system extends the envelope of operation by decreasing the expected limit loads. A control system adds additional degrees of freedom and feedback paths, and hence allows new instabilities and potential resonances.

C. Shutdown (Emergency) Systems. Shutdown systems are the special control systems used in control region (4). They are intended to limit the exposure to high wind speeds (e.g., hurricanes), loss of drive load (overspeed), or adverse environmental conditions such as icing. The system loads are primarily aerodynamic except for overspeed conditions, where inertial (centrifugal) loads may be as high as aerodynamic loads. Most such systems of interest function aerodynamically; examples include pitch feathering devices, ailerons, blade tip tabs, and spoilers.

D. Interference. By "interference" is meant the aerodynamic interference with other bodies. This category includes wakes from other turbines as well as wakes from components supporting the turbine.

1. Wake Model Development: Modeling of the wake structure and decay is important for array design and siting plans. It involves describing mathematically the wake of the turbine, and assessing the blockage and velocity decrements in the downstream turbine inflow.

\section{Component Interference} Development: Components which produce aerodynamic wakes include towers, guys, stays, nacelles, and rotor hubs. These wakes also influence the inflow to the turbine. 
In the previous Chapter, the aerodynamic study areas which are relevant to wind turbines were described. In this Chapter, these areas are discussed in relationship to the generic design approaches considered by the Panel, and prioritized in order of general importance to the goals of the federal R\&D program.

The generic design approaches are discussed in detail in Appendix 3. They represent the most probable configuration choices available now, although new approaches will appear as research is done and new products are developed. These are given in Figure 2, which shows the attributes, aerodynamic characteristics, chief unknowns and known limitations.

With the approaches defined, the Panel was able to construct a matrix of aerodynamic study areas vs. the generic approaches. A summary chart was then constructed which prioritized the aerodynamic study areas for each generic approach. This chart is given in Figure 3.

A rating scale of 0 to 3 was placed on each topic, to depict the relative need for that approach. The ratings should be interpreted as follows:

Rating: 0

Interpretation: Does not apply to this approach; or advancement in this area will not result in improvements to this approach.

Rating: 1

Interpretation: Work should be done in this area, but it should be low priority.

Rating: 2

Interpretation: Work must be done in this area, and should be high priority.

Rating: 3

Interpretation: Work should start in this area, must be done, and will result in major improvement to this approach; the highest priority.

For each generic design approach, the rankings indicate the order of importance for effort in each study area. This can be seen by referring to Figure 3. In the opinion of the Panel, substantial effort in the highest priority areas, as indicated, can result in the greatest improvement in performance and reliability for each approach.

The aerodynamic study areas, summarized in order of priority are:

\section{Unsteady Aerodynamics}

Dynamic Stall Understanding--

Develop an understanding of the 2-D and 3-D hysteresis effects of dynamic stall.

Testing Methodology for Unsteady Flow--

Stimulate complex unsteady flows in wind tunnel and total system (field) tests in order to exploit dynamic stall effects. 
Airfoil Development Studies for Unsteady Flow--

Initiate a design process which will yield airfoils specifically suited for unsteady flow, and which considers the significance of:

o dynamic stall repeatability and insensitivity

o airfoil roughness

0 delayed stall and soft stall

o performance and prediction

$o$ rotor stability, and

o airfoil control devices.

\section{Wind Inflow Models: The Development} of Aerometerology

Establish a new study termed aerometerology, which will attempt to develop realistic inflow models for the assessment of unsteady effects,including the specific cases of:

o unsteady, uniform inflow-or representation of the uniform gust front;

0 unsteady nonuniform inflow-or representation of frozen turbulence;

o steady, nonuniform inflow-or representation of steady inflow fronts; and

o stochastic inflow-or representation of inflow in the frequency/ wave number domain.

\section{Interface Topics}

Aeroelasticity--

Develop a methodology for defining the unsteady airloads which must be included in the structural dynamic models.

\section{Control Systems--}

Investigate the performance and reliability benefits to be obtained with external control actions, such as ailerons, in steady and unsteady flow.

Wake Model Interference Development--

Develop and verify a model for the structure and decay of the unsteady wind turbine rotor wake.

\section{Component Wake Interference Development--}

Develop and verify models for the structure and decay of the unsteady wakes caused by turbine components such as towers.

\section{Shutdown (Emergency) Systems--}

Develop an understanding of the behavior of airfoils under extreme conditions, and at very high angles of attack, such as a moving aileron in separated flow.

In addition, further steadystate aerodynamics studies should be continued in order to provide a suitable data base for the above 
investigations and lend insight into the new physical and mathematical models and design tools which must be used.

\section{Steady Aerodynamics}

Three-Dimensional Flow--

Assess the degree of 3-D or spanwise flow which occurs under typical conditions, and relate that to the inflow and turbine wake geometry.

Wake Modeling--

Develop and verify a model of the turbine vortex wake geometry suitable for performance and stability studies.

Effect of Roughness in Steady State--

Develop an understanding of environmentallyinduced airfoil surface roughness on airfoil transition and separation in steady flow.

\section{Airfoil Mechanisms--}

Investigate the effect of airfoil mechanisms, such as vortex generators, on delaying turbine rotor stall and separation.

Two-Dimensional Airfoil Development--

Develop a verified methodology for tailored wind turbine airfoil design in steady state to establish a database for future unsteady airfoil design.
Testing Methodology for Steady Flow--

Continue steady-state wind turbine testing in wind tunnels and in the field, to acquire long-term performance data and to investigate the benefits of new airfoils.

\section{SPECIFIC AERODYNAMIC RESEARCH NEEDS BY PRIORITY}

The highest priority research areas are the ones which "cut across the board", i.e., are high priority for each approach. Study in these areas is discussed further in Chapter 5, The Need for Basic Research. The research needs are described here in order of their priority ranking, from the highest priority to the lowest.

Work in all the areas should continue in order to provide across-the-board progress, since that is what is required. The lower priority items must have reached a level of maturity such that the more complicated, higher priority items, can be sensibly done. The key indicator of this maturity is the existence of effective mathematical design tools which have been adequately verified by experiment. None of the wind turbine aerodynamic research areas can be said to have achieved this level of maturity.

\section{A. Unsteady Aerodynamics.}

1. Dynamic Stall Understanding (2-D and 3-D Hysteresis): This is the highest priority with all the approaches (with the exception of high tip speed turbines, which are discussed below). Both 2-D and 3-D studies are essential to assess 
the present HAWT turbines, and design tools must be much better if major improvements are to be made later. The situation is particularly important for VAWT where very little is now known about the unsteady performance.

For VAWT turbines, the Panel believes that major improvement in performance is possible if unsteady flow can be exploited. 2-D dynamic stall hysteresis studies will be directly applicable to these turbines.

For HAWT turbines, the unsteady airloads which now cause unexpected failure and fatigue are of primary importance. For stall-controlled turbines, dynamic stall must be understood and sensibly quantified before any major airfoil improvement development can take place. Pitch-controlled turbines are subject to the same dynamic stall events as are the stall-controlled machines, and probably at a worse place in the operating regime from a gust turbulence point of view, i.e., in the high wind load control region 3 [16]. There is also evidence from recent studies that the pitching rate (of a pitch-control machine) can greatly influence unsteady airloading and gust response $[17,18]$.

Knowledge of the effect of Reynolds number on dynamic stall is necessary for both HAWT and VAWT in the large sizes.

The high tip speed ratio turbine is a special case. It is likely to be less susceptible to dynamic stall than the others, since it operates at lower lift coefficients which are further from the stall boundary. Also, inflow fluctuation causes correspondingly smaller angle of attack variation for high tip speed turbines (see Figure 18). Nevertheless, this turbine will still be vulnerable to inflow events which lead to unsteady aerodynamics.

2. Testing Methodology: Unsteady aerodynamic testing methodology should be developed in parallel with theoretical development. Simulating complex, difficult-to-quantify events will likely be the source of most initial advances in exploiting unsteady effects.

a. Wind Tunnel. The capability to do unsteady testing in wind tunnels exists now, and offers the chance to see and explain an unsteady event before it can be quantified. However, much remains to be learned on the boundary layer level before windtunnel studies can be routinely equated to free flight conditions (see for example, Ref. [19]). Work in this area is of crucial importance since the best chance for understanding unsteady boundary layer behavior lies in carefully-controlled wind-tunnel studies.

b. Total System (Field). Total system tests are important, just as with aircraft, and will be much more difficult than windtunnel tests. The rotor behavior and flow must be recorded, but: the field inflow must also be recorded in sufficient detail to permit inflow modeling; this is discussed further in the section below on inflow. One attribute of wind turbines is the relative ease with which a small-scale rotor can be used in field testing. This is possible since the range of Reynolds number from convenient test size to actual rotor size, even with multimegawatt machines, is relatively small.

3. Airfoil Development Studies:

a. Repeatability and Insensitivity. Once the repeatability of dynamic stall has been determined and quantified, design process will 
FIGURE 18. HIGH TIP SPEED ROTORS: EFFECT OF INFLOW FLUCTUATIONS

\section{LOW TIP SPEED RATIO}

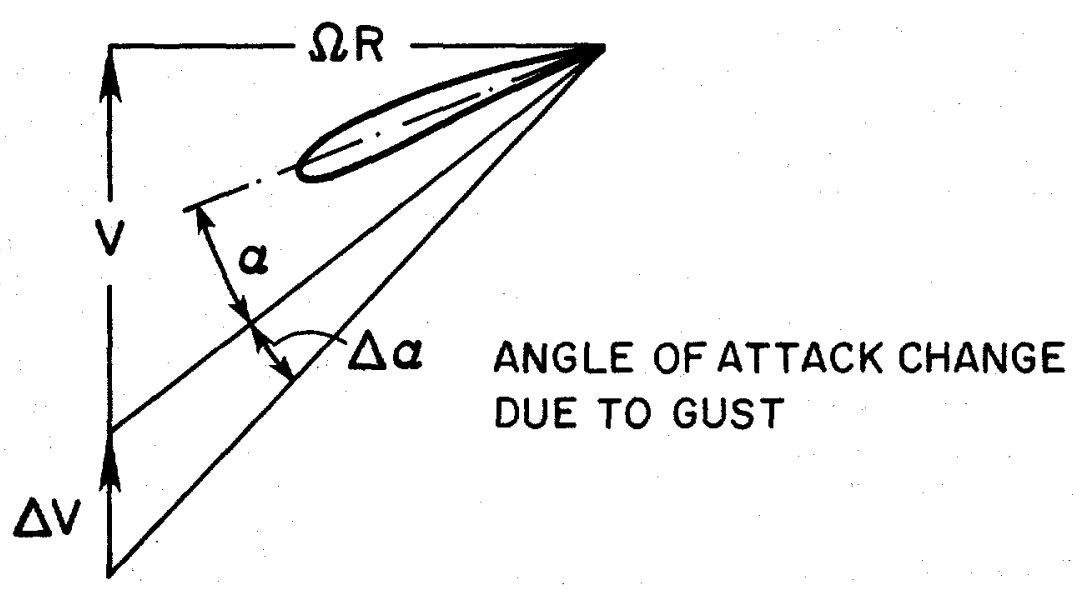

HIGH TIP SPEED RATIO

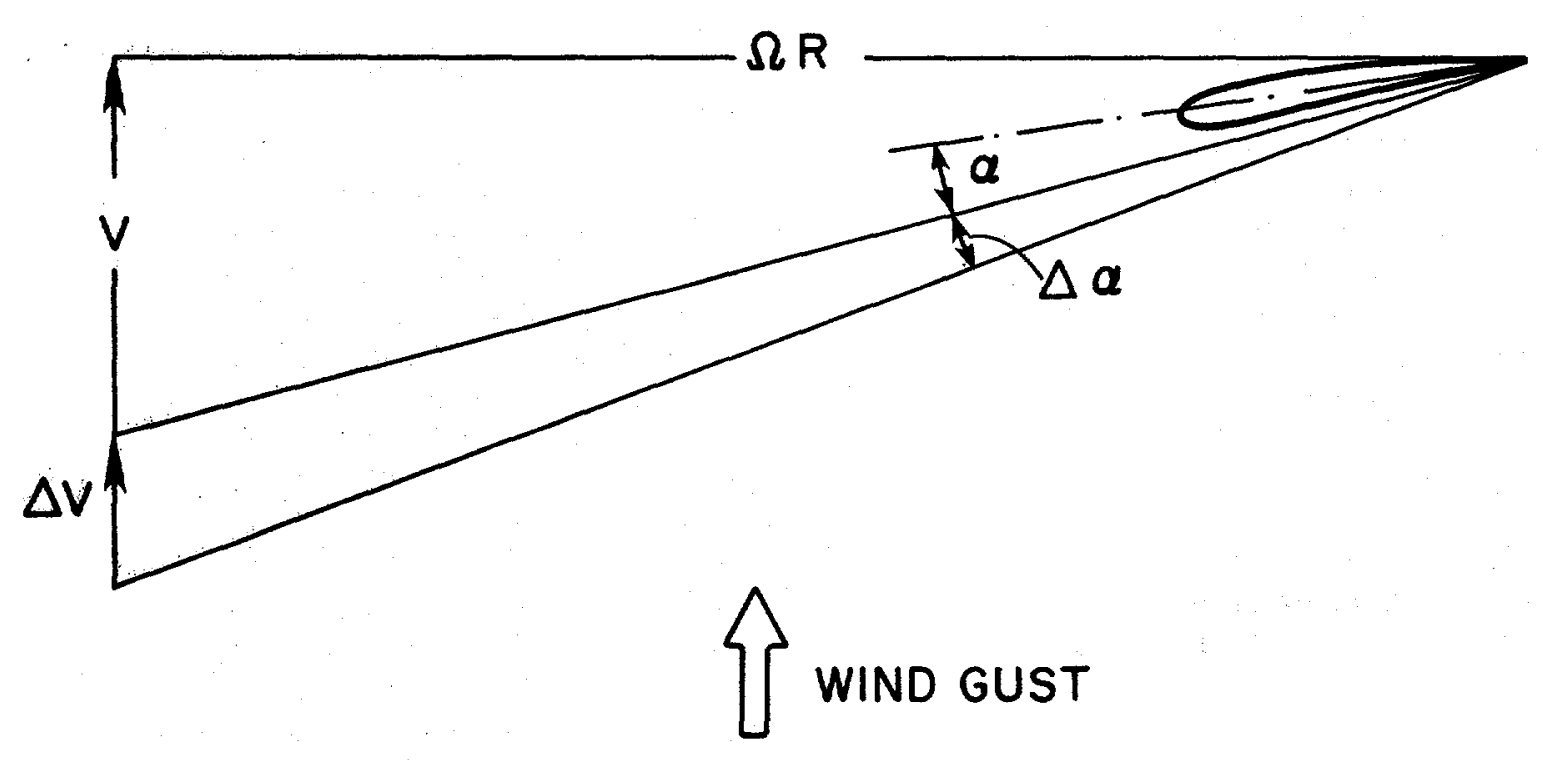


yield new airfoils and blades which are insensitive to their fluctuating time history. These new airfoils will dynamically stall in a predictable way, hopefully under a wide variety of conditions. This characteristic is of paramount importance with stall-controlled wind turbines, which operate at high lift coefficients on the edge of dynamic stall for much of their lifetime. It is important for this rotor to stall the same way each time, since stall is the only load control available.

Essentially none of a wind turbines' lifetime is spent in steady flow; and this is particularly true of VAWT, so the repeatability of dynamic stall is a starting point for improving its aerodynamics. Currently, not enough is known even to decide whether delayed stall or soft stall is needed for VAWT. Field tests show that the straight-bladed VAWT is very sensitive to its collective pitch angle, which probably determines whether the irreversible point of the dynamic stall hysteresis curve is met or exceeded [20].

b. Effect of Roughness.

All wind turbines accumulate surface roughness. Their degree of exposure and accumulation is much higher than for conventional aircraft, and wind turbines should ideally require less surface maintenance than aircraft. It is clear from steady-state tests that most airfoils are sensitive to roughness. However, these steady-state tests are likely to apply with confidence only to the high tip speed ratio wind turbine, which operates further from unsteady aerodynamics effects than other designs. Thus unsteady tests must be done.

Field experience with stall-controlled HAWTs and VAWTs has shown the profound effect of roughness on energy output and maximum lift (power rating) [21]. Figure 19 gives an extreme example of this, wherein the approximate power curves from field tests for a 13-meter diameter stall-controlled HAWT under smooth and rough airfoil conditions are given. The steadystate stalling behavior of airfoils exposed to varying degrees of roughness has received, and is currently receiving, a lot of attention by the sailplane and wind turbine engineering communities $[22,23,24]$. It is not clear what the unsteady behavior will be. This makes it imperative that the unsteady tests be done for those airfoils if they are to be considered for wind turbines.

c. Delayed Stall and Soft

Stall. When unsteady aerodynamics has been sufficiently developed, modifications to the dynamic stall characteristic can be made by deliberate design in order to improve performance of wind turbines. Soft stall may lessen the fatigue exposure at the cost of increased output; delayed stall might permit more energy capture at the cost of high peak loads.

Any improvement in dynamic stall for VAWT affects the entire operating envelope of the turbine, and not simply extreme or special turbulence bands, as is the case for HAWT. Therefore, VAWTs stand to gain a great deal if dynamic stall can be made to work for them.

The uncertainty about the desired dynamic stall hysteresis shape for VAWTs also exists for stall-controlled rotors, whether HAWT or VAWT. Delayed stall has the advantage of high momentary lift (and torque) forces, but the disadvantage of high impulsive airloads. Soft stall has the 
FIGURE 19. EFFECT OF AIRFOIL ROUGHNESS ON POWER CURVE

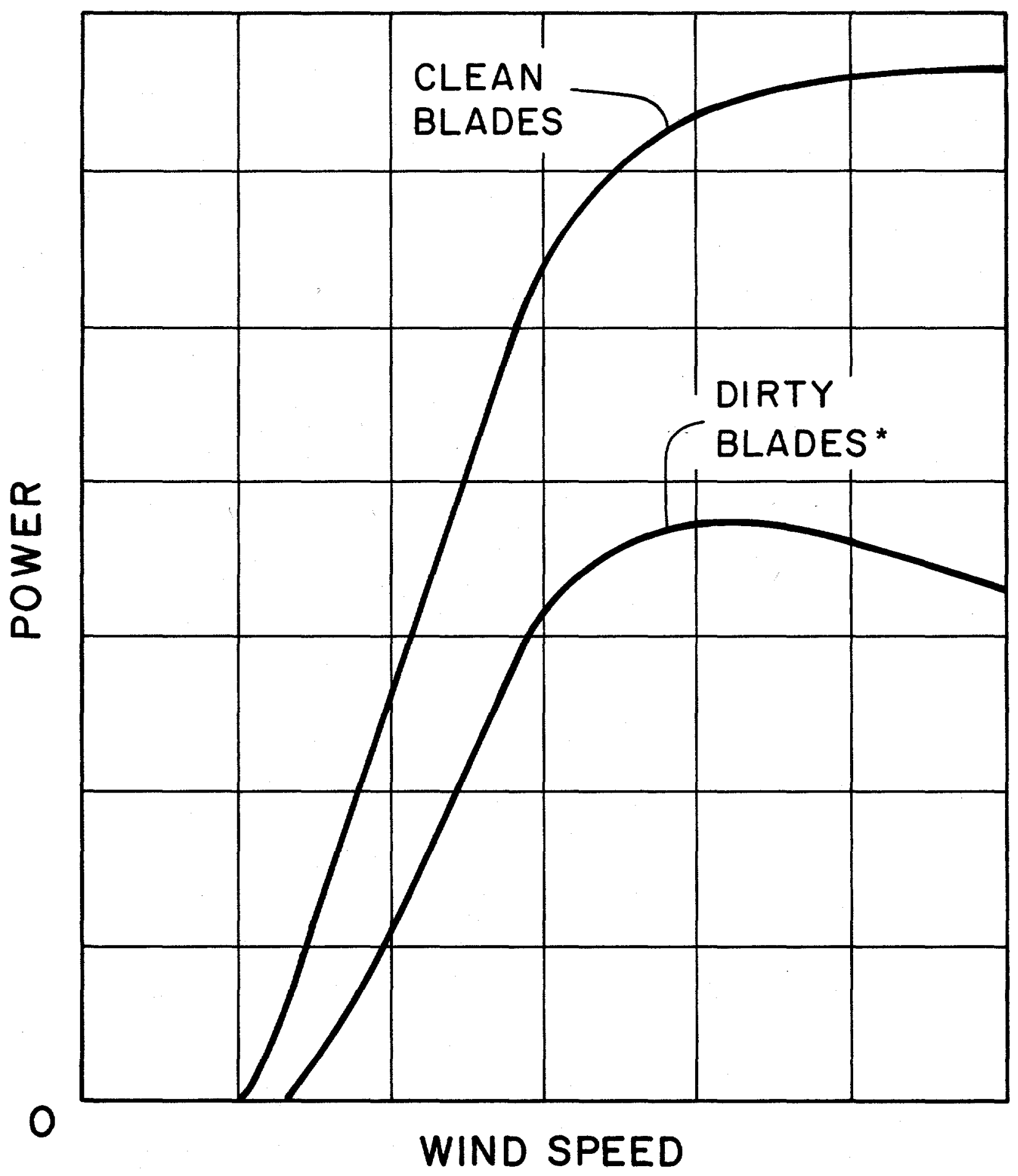

* This represents an extreme example 
advantage of no increase in fatigue loading, but the disadvantage of loss of beneficial aerodynamic damping to motion and increased torque.

The same arguments for delayed stall and soft stall apply to pitch-control and high tip speed rotors. However, the high tip speed rotor has a lower priority by virtue of its lower exposure to dynamic stall conditions.

4. Prediction of Performance: The next logical priority is combining the rotor simulation and the unsteady aerodynamics airload calculation to predict the unsteady rotor performance. This includes primarily analysis of the unsteady vortex wake and its feedback effect on the induced velocity field which determines rotor angle of attack.

For the VAWT, the distorted, unsteady vortex wake probably has a much greater influence on the rotor performance than other inflow perturbations. Initial test data tend to confirm this [25]. It is unlikely that confident performance, either unsteady airloading or torque output, will be successfully predicted for VAWT until the unsteady aerodynamics is much further developed. In contrast with HAWT, there is no mathematical coordinate reference frame for VAWT in which the flow can be approximated as steady state. The progressions of wake models, from the simplest to the complex, are very different for VAWT and HAWT, and probably different mathematical tools will be needed.

For HAWT, the study of the unsteady wake will enhance performance prediction, and also allow mathematical description of the wake physics for wake interference development study.
5. Rotor Stability: Of next importance is the stability of the rotor system, which can be analyzed when the unsteady wake has been adequately assessed. This is clearly important for free yaw HAWTs, which have a profound requirement for dynamic stability of the operating rotor under unsteady conditions. Only slight off-tracking in yaw can result in loss of up to half a turbine's annual output $[26,72]$.

For HAWT, the mathematical model, and code, will generally be different for the stability calculation than the performance calculation, since the stabilizing airloads are in the flapping (lift) direction, and the power-producing airloads are in the lead-lag (torque and drag) direction.

It is generally agreed that it is possible for any HAWT to be made yaw-stable in steady flow, either upwind or downwind of the yaw axis, with proper blade design [27]. However, unsteady flow can cause selective dynamic stall on opposite sides of the rotor disk, creating a destabilizing yaw moment (see Figure 20), Yaw damping used to limit yaw rates for blade load control might aggravate the yaw stability under these unsteady stall conditions. Such behavior has been observed on operating wind turbines [26].

For the VAWT, yaw stability is not a concern since the VAWT is insensitive to wind direction. One possible exception is the straight-bladed VAWT with cyclic pitch control, which requires an inflow direction or stagnation locator to phase the cyclic pitch input properly (Figure A-13). This should be classified in the controls category below. 
6. Airfoil Control Devices: These are distinguished from the devices which will probably be employed in the airfoil development studies above, in which a leading edge slat, for example, may be used to create tailored dynamic stall. Those devices are considered passive since they will likely be in use during most of the operating time. Here we are concerned with actuating devices which are used only in certain conditions, such as flaps on conventional aircraft during takeoff and landing, and can be called active in terms of deployment.

The clearest applications of add-on airfoil devices, such as flaps and slats, are for increasing VAWT performance and exploiting small extensions of the operating envelope on low tip speed ratio HAWTs which have large blades. It is not clear which devices will be useful or under which operating conditions a high lift or stall delay device might be employed.

B. Inflow Models. The prioritizing of the levels of complexity in inflow models is straightforward.

All the HAWTs have the same need for a realistic inflow representation. This must be done before the unsteady effects can be assessed for each operating approach. Then the relative importance of dynamic stall can be determined. Probably the inflow frequency ranges, for instance, between large and small diameter rotors, will vary, but nothing can be said at this stage with confidence about those ranges.

The situation differs for the VAWT, which is probably an order of magnitude more vulnerable to its own self-generated unsteady effects than to the unsteady effects of inflow.
This discussion can be thought of as the first level, or first order investigation in a new field of study which the Panel has termed aerometeorology, and which is discussed further in Chapter 6.

1. Unsteady, Uniform: The highest priority is for study in the simplest unsteady frame, that is, a uniform gust front. Atmospheric turbulence can be intuitively decomposed into high-frequency events which are "smaller" than the rotor and result in degrading airloads over part of the rotor disc, and low-frequency events which are "larger" than the rotor and represent potential capturable energy. This is depicted in Figure 14.

The "large" fluctuations are to first approximation in this unsteady, uniform category. It is clear that the rotor diameter is important, but the relevant scale parameter of the inflow cannot be determined until more is known about the conditions which precipitate dynamic stall. It is likely that rate of change of angle of attack will have a dominant influence: it is directly proportional to the initial shape of the uniform gust front (sometimes called rise time or slope in the literature; see Figure 12). Obviously, the duration of the gust, along with the dynamics of the rotor and the frequency response of any pitch change, determine whether the event will cause momentary airload changes or result in increased energy captured.

2. Unsteady, Nonuniform: The concept of frozen turbulence allows spatial and time variation of the inflow front. Taking one idealized eddy or fluctuation, and following it through the rotor 
FIGURE 20. YAW STABILITY

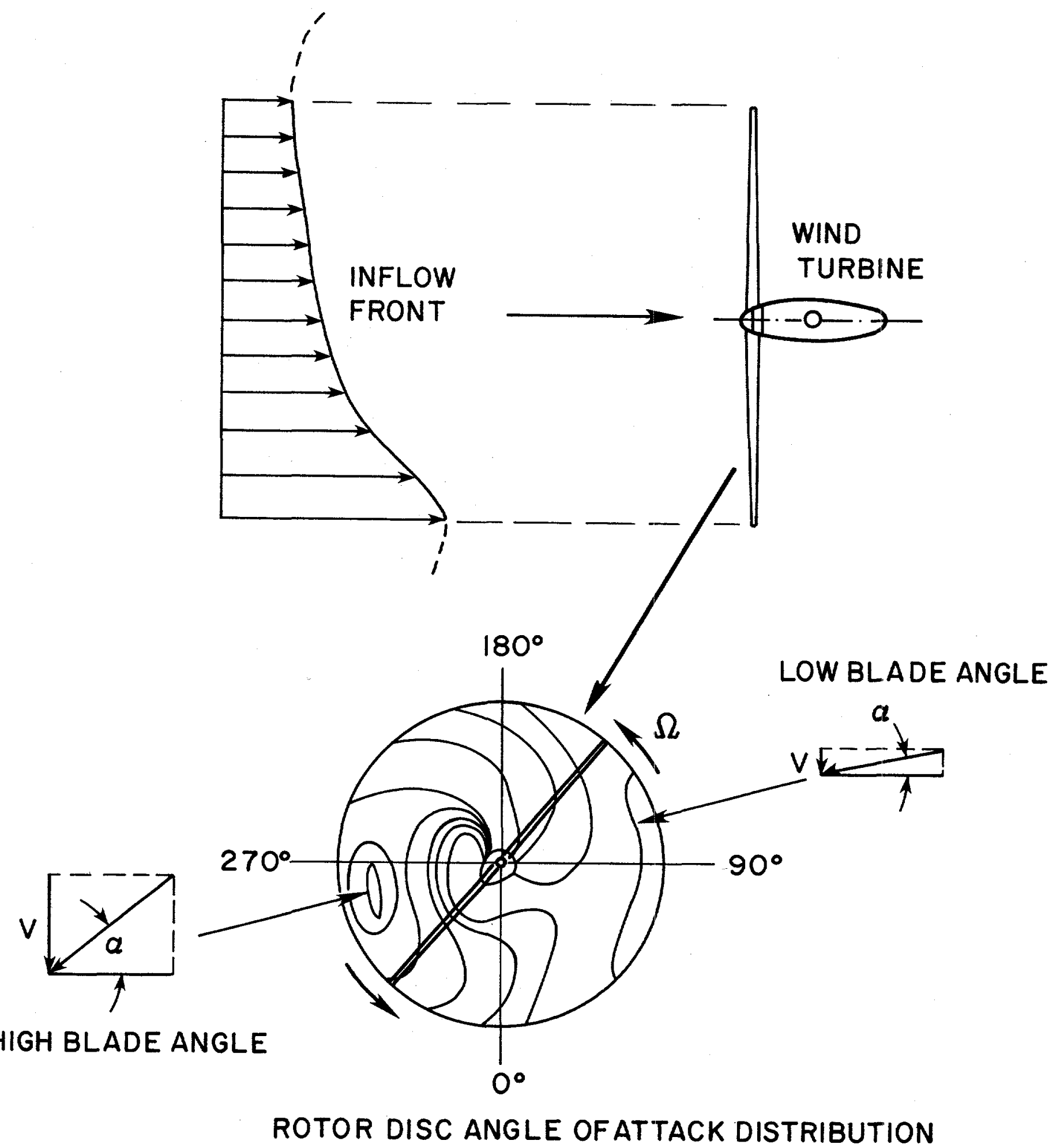


plane, Figure 21 depicts the increase of blade angle of attack on one side of the fluctuation (advancing side) and the decrease of angle of attack on the other (retreating side). It is clear that the slope of the angle of attack curve (rate of change), and the "static" value at the initiation of the fluctuation, determine whether unsteady effects will occur. Instead of the entire rotor being subject to the unsteady effect equally, as with a uniform front, here only part of the rotor is vulnerable.

To differentiate between a "uniform" gust front which envelops the entire rotor and the "nonuniform" front which selectively affects the rotor disk, the Panel suggests that field testing employ concentric rings of suitable anemometers. Figure 22 depicts such a field testing arrangement. Inflow data taken simultaneously at many stations and heights on the concentric rings will describe the scale of the fluctuations which pass through the rotor plane, causing unsteady airloading or captured turbulent energy at the wind turbine. Both the spatial variation (nonuniformity) and time variation (unsteadiness) must be recorded.

3. Steady, Nonuniform: The area of inflow investigation of next highest priority is steady, nonuniform flow. The simplest example of this is linear inflow shear. There is no change of the inflow front with time; but there is, however, unsteady flow relative to the rotating blade and its component airfoil sections. Consider a simple linear shear inflow. It is clear that the normal shear flow can be represented as an average uniform component with a superimposed linear shear (Figure 23). The shear induces a once-per-revolution sinusoidal variation in blade angle of attack, which can be verified in the blade element diagrams of the figure. The unsteady flow observed in the rotating frame of reference on the airfoil occurs at the rotor passage frequency, which is in turn the first harmonic of a Fourier series.

It is an easy extension of thought to visualize the other integer harmonics of the rotor frequency, namely 2-per-revolution, 3-per-revolution, etc., being caused by more complex inflow fronts. This is the mechanism by which the rotational harmonic spikes appear in the turbulence spectral density curve as seen by a rotating blade; see Figure 24 [28]. It is simply a manifestation of the change in reference coordinate system from stationary to rotating. The importance of this to the aerodynamic investigation of wind turbine blades is that even in steady inflow, unsteady airloads on the airfoil will result. It is not yet clear which inflow fronts or harmonics are most likely to precipitate unsteady aerodynamic events on the blade.

Steady, nonuniform flow may be the most practical representation of terrain and interference effects. It is possible that the variation in complex terrain flow and interference wakes will of ten be so slow as to be steady-state relative to normal atmospheric fluctuations. The first-order yaw stability of free yaw turbines may be reducible to a tractable steady flow problem if the inflow can be defined in this way.

4. Stochastic: It is clear that stochastic evaluation techniques can be very powerful tools when the inflow is suitably mathematically modeled. A frequency or harmonic 
FIGURE 21. CONCEPT OF FROZEN TURBULENCE

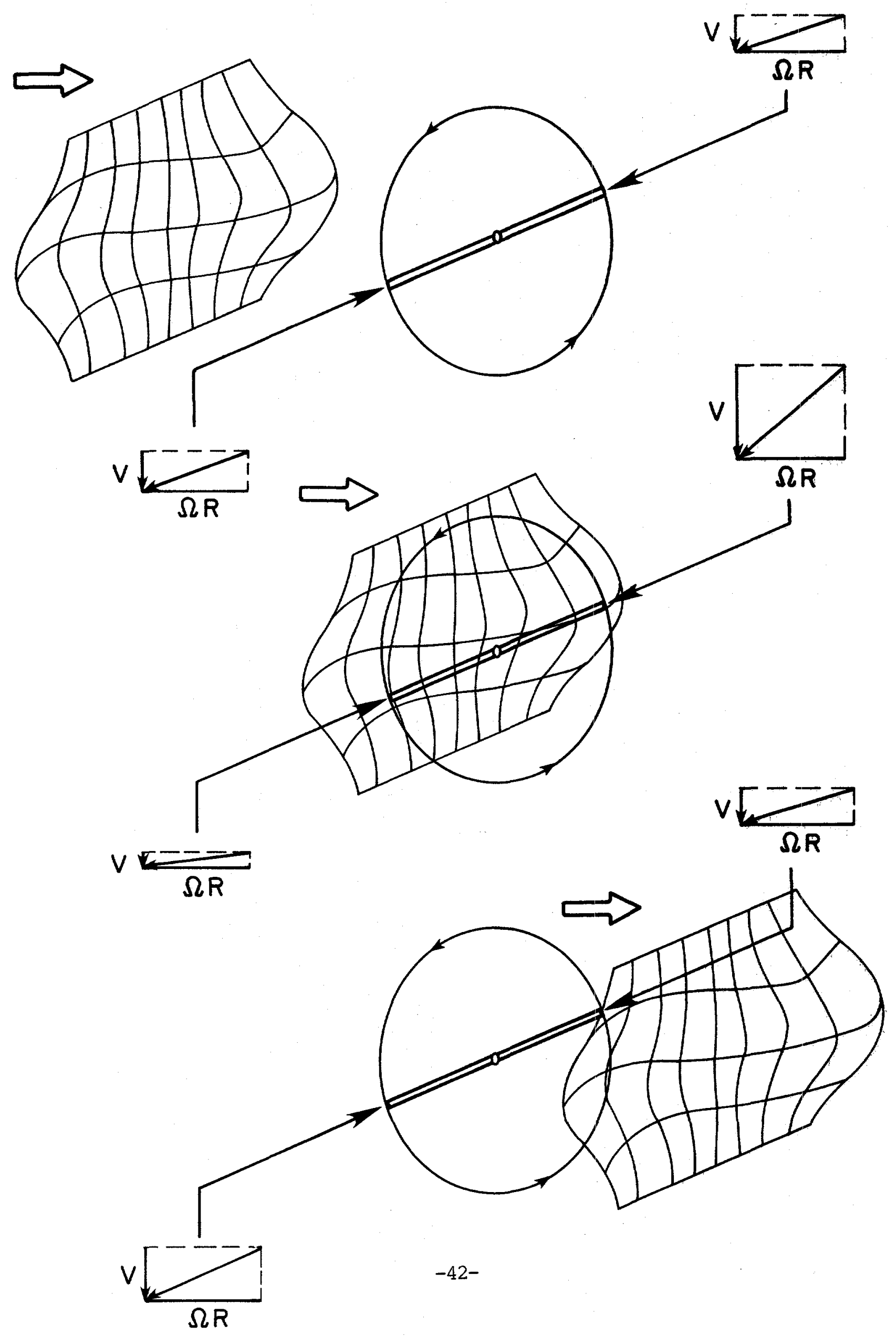




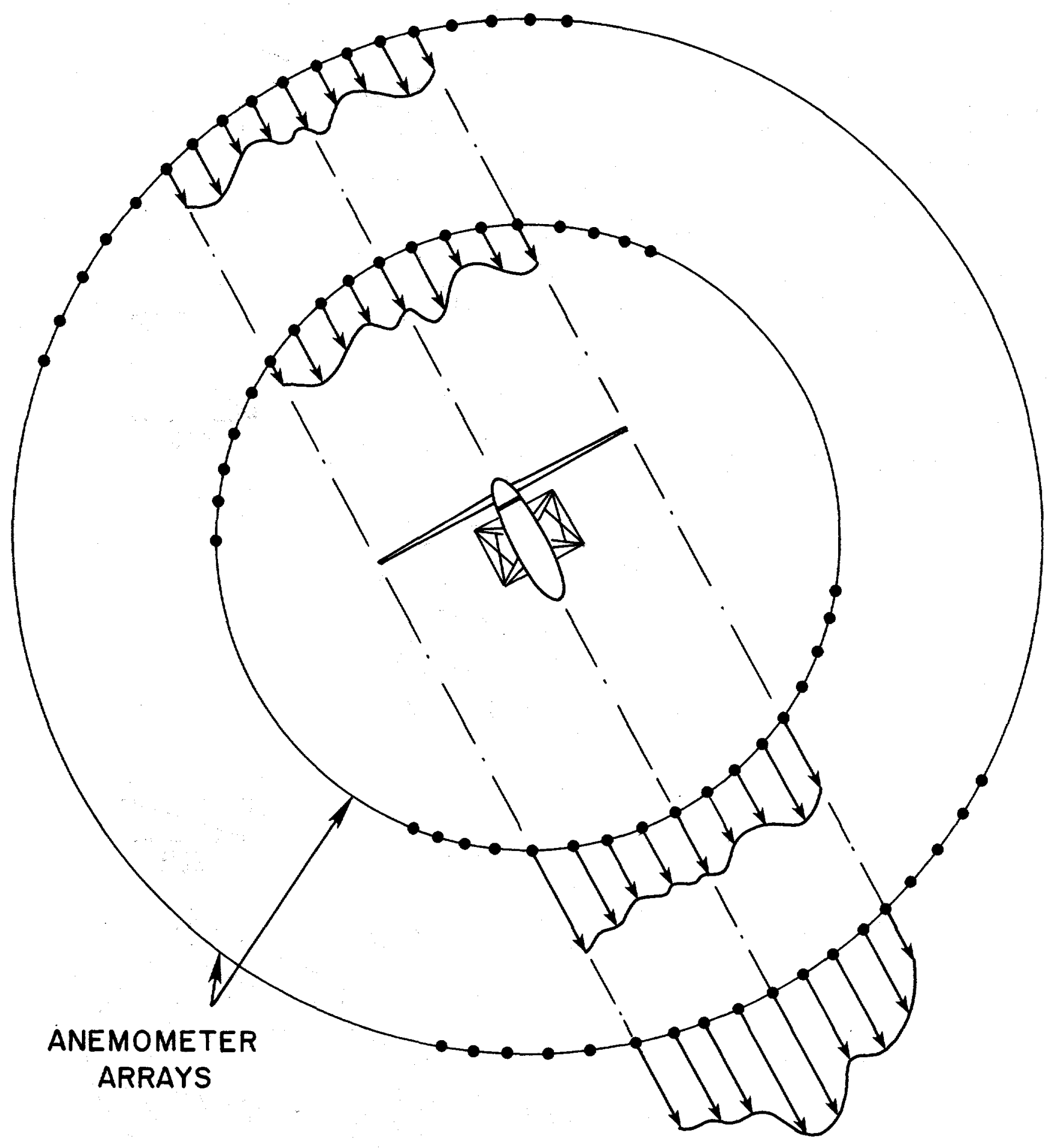



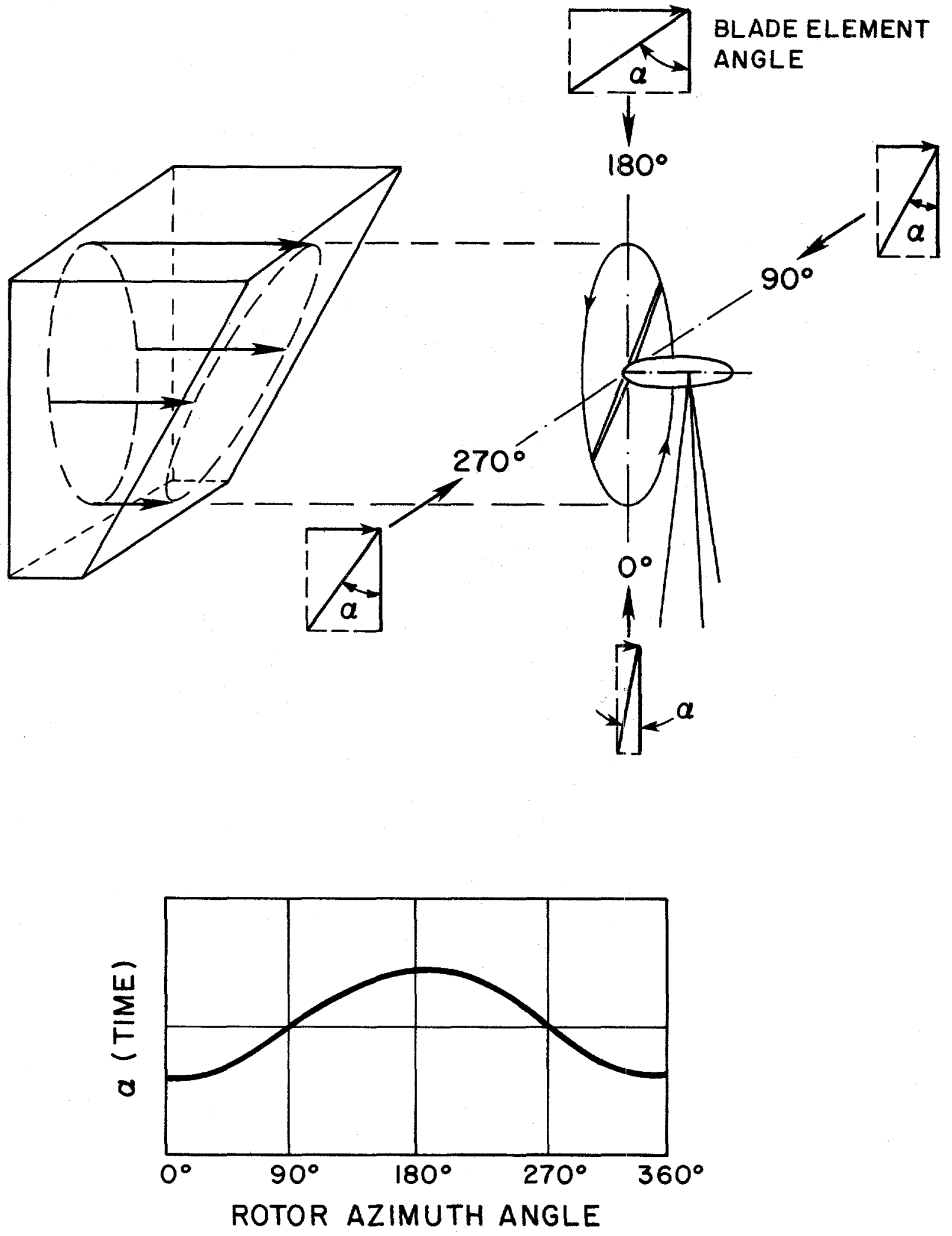

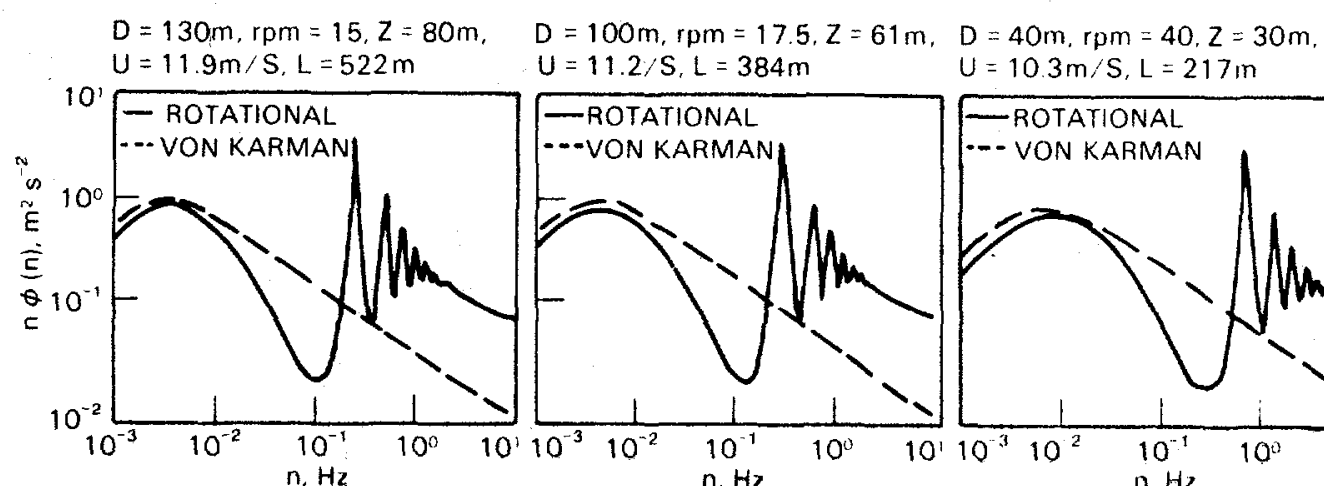
$U=11.2 / S, L=384 \mathrm{~m}$ $U=10.3 \mathrm{~m} / \mathrm{S}, \mathrm{L}=217 \mathrm{~m}$
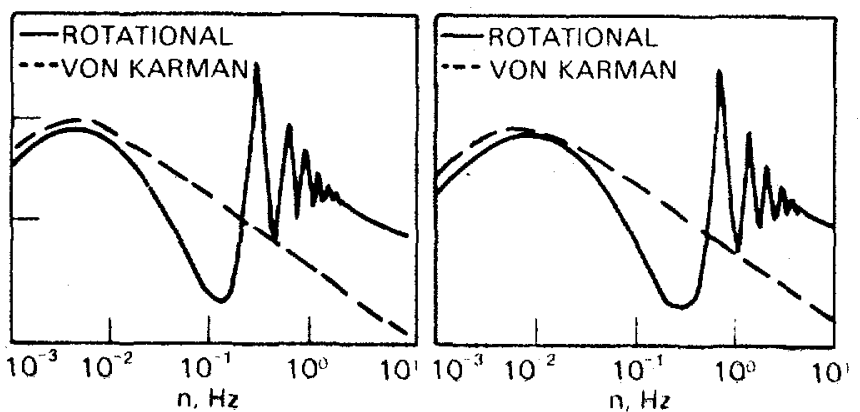

$D=10 \mathrm{~m}, \mathrm{rpm}=60,2 \approx 20 \mathrm{~m}$

$D=4 \mathrm{~m}, \mathrm{rpm}=240,2=15 \mathrm{~m}$.

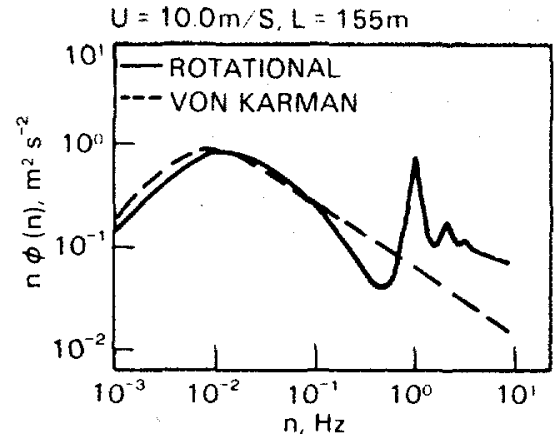
$U=9 \mathrm{~m} / \mathrm{S}, \mathrm{L}=117 \mathrm{M}$

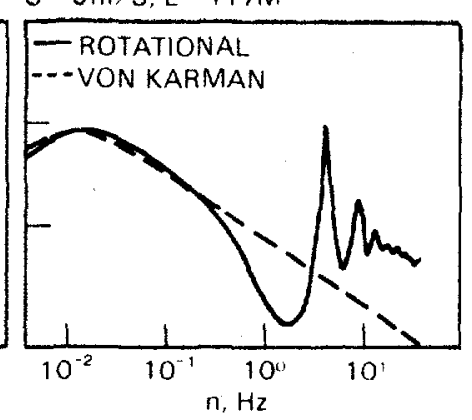

Theoretical spectra for rotationally sampled wind speed for five sizes of wind turbine. The dashed curves represent Eulerian spectra. The smallest turbine is $4 \mathrm{~m}$ in diameter; the largest is $130 \mathrm{~m}$. 
seems the most productive long-range mathematical strategy to use in the inflow (aerometeorology) study, since it also allows frequency domain analysis. Stochastic analysis can be invaluable in a number of specific ways since it permits:

- Representation of many data points taken in field tests.

- Rapid evaluation of fatigue loading.

- Comparison of hypothetical results with historical field data.

The Method of Bins [29] is a simple application of stochastic methods to wind turbine field performance. In order to be effective, stochastic methods must be related to theoretical predictive models as well as to field data. Only in so doing can general conclusions be drawn, and anything more than an ornate description of complex unsteady events be accomplished.

A possible early application of a stochastic approach will be in representing the timewise variation of the inflow, that is, the unsteadiness of the flow as the front travels along. This could be a probability distribution, or the likelihood of experiencing a given spatial nonuniformity in the inflow. In this example the spatial variation, e.g., the shape of the front, would still be deterministic, perhaps a Fourier series since the frequency and wavelength concepts are so physically valuable. In short, the character of a time function would be superimposed on a spatial representation written in harmonic series. This is depicted in Figure 25.

5. Steady, Uniform: It could be argued that steady, uniform inflow is not relevant to this discussion, and that this category represents the stereotypical thinking which must be abandoned in order that the rest of the inflow studies can bear fruit.

The only wind turbine approach for which steady uniform inflow is considered presently adequate is VAWT, which requires considerably more emphasis on the unsteady inflow caused by its own motion. Clearly, the above discussion of unsteady inflow also bəars on VAWT, and should eventually be considered after the more pressing studies are done.

C. Interface Topics. These are the wind turbine aerodynamic research needs for interdisciplinary studies involving wind turbine systems.

1. Aeroelasticity: As wind turbines become more structurally efficient and lighter, the importance of aerodynamic loading relative to inertial and gravity loading will be higher. At present, most successful turbines operate at low tip speed ratio and are intentionally heavy and stiff so that inertia and gravity dominate the load spectrum. In this way they minimize the impact of the unsteady aerodynamics to which they are subjected (see Appendix 2). When inertial, structural elastic, and aerodynamic loads all enter, aeroelastic studies become important, and there exists more opportunity for instabilities and adverse resonance. The highest priority research need in the area of aeroelasticity is the definition of the unsteady airloads which force the dynamic mathematical model.

For stall-controlled rotors the most likely phenomenon is stall flutter, which has been documented in a large wind turbine [30]. It is likely that stall flutter defines an 
FIGURE 25. COMPLETE WIND INFLOW REPRESENTATION

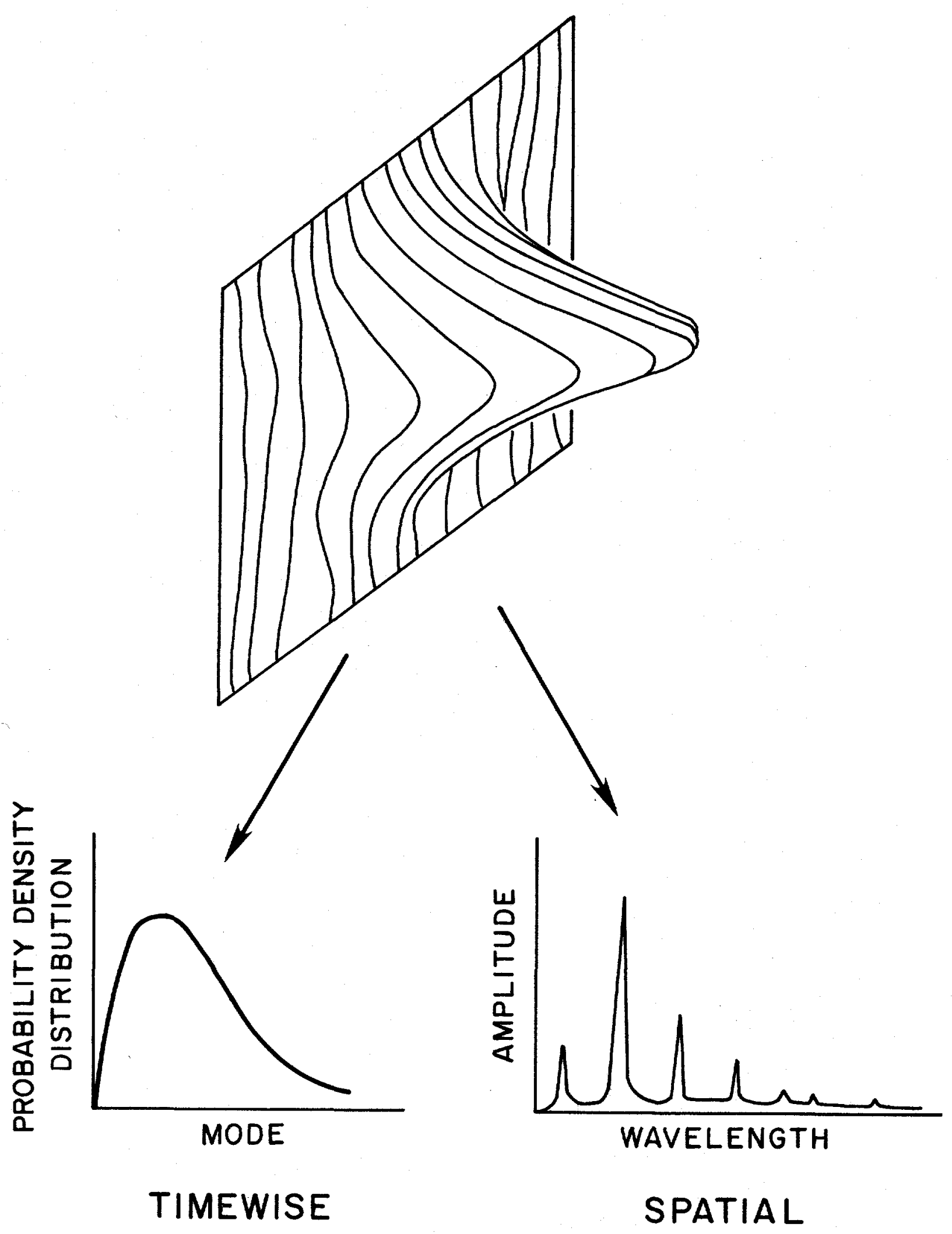


upper limit for the size of stallcontrolled rotors with stiff, cantilevered blades $[30,31]$, and that use an airfoil that exhibits a normal loss of lift at stall. Partly for this reason, there is interest currently in the design of wind turbine airfoils which do not have this normal drop in CL-max at stall, but instead maintain a nearly constant lift coefficient after stall [32].

2. Control Systems: Here the concern is with external control actions, such as pitch change, which can be programmed and actuated according to a preset strategy or algorithm. This research area interfaces with automatic control technology, and again the research need is to provide unsteady airloading for the system control algorithms. Control systems add complicating degrees of freedom in damping, and instabilities to the system dynamics. Control actions also result in induced unsteady airloads which feed back into performance and rotor stability.

The ultimate control system would be an airfoil shape which automatically optimized the unsteady flow and shed unwanted loading. Some of this may ultimately be achievable for certain restricted applications, such as might be possible with soft stall for stall-controlled rotors.

Control systems for pitch-controlled rotors (including partial span pitch) determine the proper average angle of attack for given conditions. Thus the operating envelope can be extended, and performance consequently improved. Pitch change on a constant speed rotor can be viewed as a method for maintaining constant angle of attack on the blade. Variable speed turbines, which use load control to operate at constant tip speed ratio, also maintain constant angle of attack by adjusting their speed. This is shown in Figure 26. It is clear that, given unsteady inflow, the time required to readjust blade conditions to the optimum depends on the pitching rate for the constant speed rotor, and rotor inertia for variable speed. It is not clear which "readjustment" approach results in higher performance or reduced momentary loading. Research is needed to define the unsteady behavior well enough that such design questions can be answered.

Aileron control surfaces, as a weak substitute for blade pitch change, can be used to control aerodynamic load by spoiling, or to delay stall [33]. The study of such aerodynamic control motions must include unsteady flow conditions as well as steady flow. Thus, there is a current need for investigations of control surface motions superimposed on the unsteady airfoil, and their combined behavior.

\section{Wake Model Interface}

Development: Modeling the structure and decay of the unsteady turbine wake is of high priority for array siting. The literature [34] shows a test example where, in a low-turbulence wind tunnel, the power decrement at 10 diameters downstream in steady flow was $40 \%$ for a representative turbine. Field experience in arrays has not been as severe [26]. This topic was given a moderately high priority by the Panel since the siting benefits could be large. 
FIGURE 26. PITCH CONTROL VS. CONSTANT TIP SPEED RATIO (VARIABLE SPEED)

VARIABLE PITCH

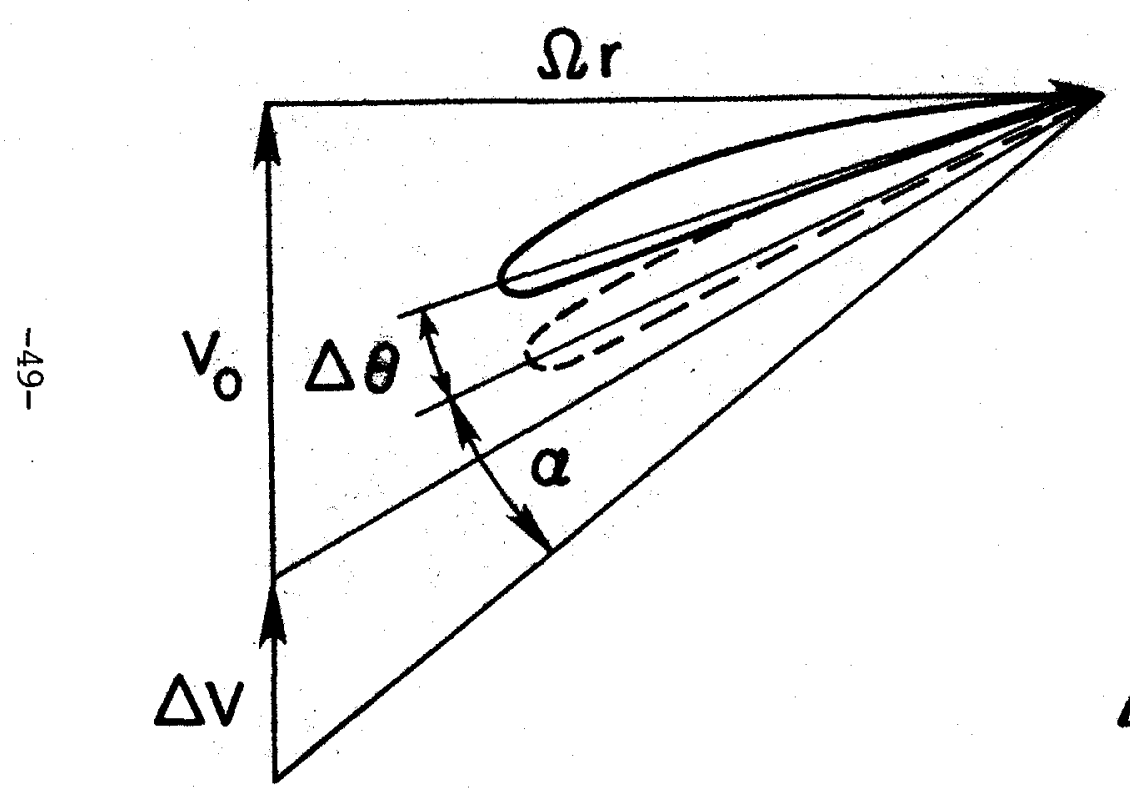

VARIABLE SPEED

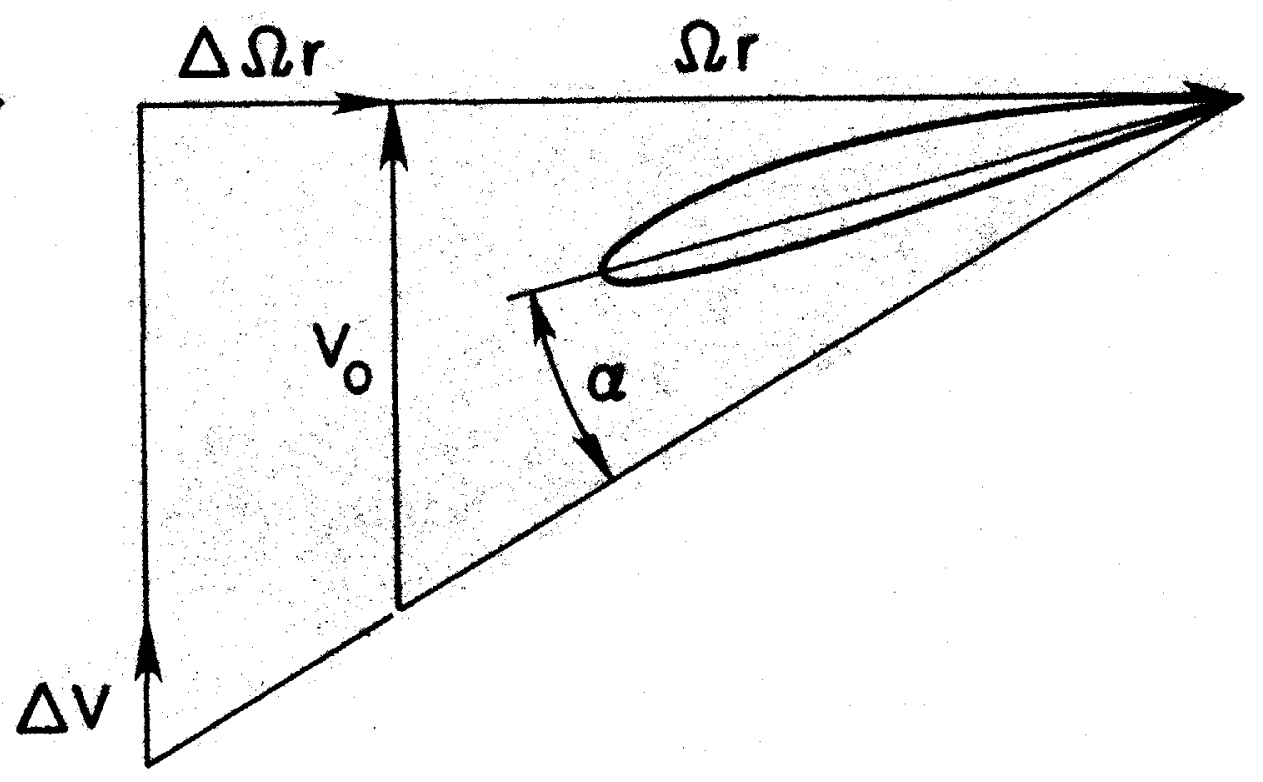


Independent theoretical [35] and experimental [36] studies of turbine wakes should continue. The critical step, however, is the development of a suitable mathematical description of the wakes within the constraints of sensible unsteady nonuniform inflow methodology. Only in so doing can the effects of turbine wakes be confidently assessed.

\section{Component Wake Interface} Development: Modeling of other aerodynamic wakes is important, but probably not as important as the turbine wake interference problem. It is imperative here also to describe the component interference in mathematical terms which are compatible with the other mathematical models.

The most important interference wakes to study are those which appear as unsteady inflow in the rotating system. The most obvious example is the tower wake for all VAWT and for downwind HAWT rotors. This wake has been shown to be a significant source of fatigue on large downwind turbines [37], and this may ultimately limit their size. As understanding and confidence in the prediction of yaw stability of rotors improves, there should be less incentive for placing rotors downwind of their towers.

\section{Shutdown (Emergency)}

Systems: The aerodynamic shutdown (e.g., high inflow, overspeed, out-of-balance) systems fall into this category. The technology need is to understand the behavior of airfoils under extreme unsteady conditions and at very high angles of attack, such as a moving aileron in separated flow. There is recent evidence that ambient turbulence levels of the inflow can affect even the performance of airfoils in fully separated flow [38], which makes this problem even more difficult.

Pitch-controlled rotors are usually considered to have automatic shutdown capability by virtue of blade pitching (feathering).

For most stall-controlled rotors, however, blade pitch is fixed, so a loss of driven load will cause the turbine rotor to overspeed until it reaches the "zero-slip" zero torque condition (see Appendix 2). Since the pitch angle is fixed at a relatively flat position for reasonable performance in normal flow, the thrust (lift) on the blade under overspeed conditions is very high. The turbine will be in Turbulent Wake State or Vortex Ring State, in which very high thrust coefficients are produced. This condition is the most difficult to analyze, and probably represents the extreme aerodynamic loading for any rotor. An aerodynamic overspeed protection or shutdown system may be nearly impossible to analyze in these conditions.

No estimate can be made at this time of the likelihood of shutdown conditions occurring for any particular turbine generic approach. The most that can be said is that the fixed-pitch, usually low tip speed ratio, stall-controlled, HAWT and VAWT rotors are more dependent on these control systems, and some improvement in their understanding is possible.

D. Steady Aerodynamics. The lower priority topics are in steady aerodynamics. They have lowest priority for VAWT turbines, since the flow never even approaches steadiness. However, there are steady-state study areas that will benefit HAWT rotors. Largely because these areas are understood 
in the traditional sense, and are tractable mathematically, substantial work in all those areas is currently underway, and should be continued. But major payoffs in performance and reliability will not come until sensible progress is made in the unsteady areas. Developments here in these steady-state areas will facilitate that by providing a database for comparison and new attempts at mathematical modeling and design tools.

1. 3-D Flow: 3-D rotor flow in steady state has moderate importance for conventional rotors, such as pitch control, variable speed, and low tip speed ratio, especially with airfoil devices.

HAWT rotors operating at high lift coefficients and especially if inboard blade sections are already fully stalled, will have radial pressure gradients which can cause spanwise flow. This has often been observed on low speed wind turbines and is currently receiving attention $[39,40]$.

In steady state the effect of 3$D$ flow is to delay stall; in unsteady flow the effects are unknown. Therefore, a technology need is to assess the degree of spanwise flow which occurs under typical conditions, and relate that to the inflow. It is likely that the steady-state approximation will not hinder this study at first, since few field tests or wind-tunnel studies have been done on the subject (see below). It is clear that the degree of spanwise flow will have some influence on the unsteady airfoil effects, but the spanwise flow studies must quickly turn to unsteady effects to be realistic.

Stall hysteresis (steady state) of a candidate wind turbine airfoil has been recently shown in a windtunnel test to be dependent on aspect ratio [41]. It is also clear from the body of literature of low Reynolds number airfoil work stemming from sailplane studies that Reynolds number is also a strong parameter that affects stall behavior in a 3-D flow.

3-D flow on a blade can also be caused by a wake vortex standing near the airfoil. Again, its effects on the induced velocity distribution should probably be modeled in steady state first for expediency, before the unsteady work is done. This study area most clearly affects turbines with strong vortex wakes (low tip speed ratio types) and those vulnerable to wake stability (free yaw types).

2. Wake Modeling: Of moderate importance is the modeling of the wake in steady flow. Some work must be done here before the unsteady wake modeling can begin. Confident prediction of performance cannot occur, however, until the wake is adequately modeled in unsteady conditions. It is of ten suggested that lifting surface theory be used to improve performance calculations over the current blade element/lifting line approach [42], but clearly, this approach due to its steady-state assumptions, may not improve performance calculations for unsteady flow.

Modeling the steady-state wake will also facilitate the stability study of free yaw turbines. It is logical to predict the static aerodynamic yaw stabilizing moment first, before more complicated unsteady wake stability is attempted. Work on this has already been started, towards the calculation of the free wake geometry $[43,44]$. 
3. Effect of Roughness: The real technology need here is to understand the effect of environmentally induced airfoil surface roughness on airfoil transition and separation. This is clearly a high priority unsteady aerodynamics topic. However, in steady state, work is still necessary as well to prepare a database for the unsteady dynamic stall studies.

Historical tests have shown the profound effect of airfoil roughness on the transition of the boundary layer from laminar to turbulent and ultimately on separation, but these data have not been fully applied to the wind turbine field test results [11]. There is qualitative evidence that the extreme roughness sensitivity of the NACA 230XX airfoil experience in the field (see Figure 19) may be due to simple underestimation of the sensitivity of the region of upper surface high pressure to leading edge roughness. That is, the "clean" airfoil in the field was able to support a high pressure distribution, and a corresponding high maximum lift coefficient, but the only slightly "dirty" airfoil had this drastically reduced. This is no doubt partially due to the vulnerability of the "droop nose" of the 230XX to bug buildup [26].

Thus, there is a technology need to study the steady roughness effects from the field tests and in wind tunnel tests in order to begin to understand the effect on transition of the boundary layer and incipient stall. In addition, the benefits of steady vs. unsteady studies of roughness cannot be assessed until proper inflow work has been done to quantify the degree of unsteadiness of the flow.

For very high tip speed ratio turbines, the flow is closer to steady state because of the lower operating lift coefficients and lessened fluctuations in angle of attack (see Appendix 2). Here, the effect of roughness on rotor performance can probably be confidently assessed with 2-D analysis. Another compelling reason to study roughness effects for these rotors is that the high tip speed rotor will likely be very low solidity and employ thin airfoils for higher L/D's and lower noise, and it is well known that thin airfoils are much more vulnerable to vagrant roughness than thick airfoils.

4. Mechanisms: This category includes high lift devices, vortex generators, flow energizers, separated flow controllers, and attachment promoters. For flight vehicles, all are an attempt to extend the flight envelope slightly by delaying stall and separation, usually on a control surface, and all carry a slight drag or performance penalty in some other part of the operating envelope. Currently the design of these components is usually done for a steady flow condition. Therefore, for wind turbines, which operate in unsteady flow, the steady-state analysis of these devices must be considered of low importance, and might properly be reserved for the blade geometry (twist and taper) compromise stage of the industry design studies, and not appear as a separate research item.

Studies of these devices may have application to turbines which operate at high lift coefficients and which could benefit from small extensions of lift during certain conditions [45]. The most obvious candidate for this study is the low tip speed ratio, high solidity turbine that has adequate chord 
and structure to accommodate such devices. However, the degree of potential improvement cannot be assessed until the unsteady inflow is better known.

5. 2-D Airfoil Development: It is difficult to justify major further 2-D steady-state airfoil studies, either theoretical or experimental, before more is known about the unsteady behavior of the new airfoils. However, it is clear that productive work is going on, and will prove to be valuable. Current lines of development in wind turbine airfoil $R \& D$ are:

1. the fixed-wing airfoil applications (e.g., LS-1 series [5]),

2. the empirical low Reynolds number airfoil families (e.g., Wortmann FX84W series for stall-controlled wind turbines [32]), and

3. the DOE-sponsored theoretically derived wind turbine airfoil families (e.g., Tangler-Somers Eppler code families [6]).

Leaving aside the roughness question for a moment, the latter two directions both show major possible improvements over the present common wind turbine airfoils, the NACA $230 \mathrm{XX}$ and $45 \mathrm{XX}$ series. It is not clear that their predicted steady-state benefits will carry over into unsteady flow. Work should continue in the above airfoil development directions in order to establish a steady-state database from which to understand and compare the unsteady behavior yet to come.

Therefore, the straightforward extension of fixed-wing airfoil design technology one step further by varying the type of airfoil along the blade, and studies on the applications of laminar and supercritical conventional fixedwing steady airfoils to wind turbines are premature until the unsteady effects are better understood.

6. Testing Methodology: Steady-state testing should continue in order to acquire more long-term average data on performance, and to investigate the high angle of attack and stall behavior of the candidate airfoils. Recent wind tunnel work [24] has pointed out the inconsistent and poorly understood stall behavior of a NACA 23024 airfoil at representative wind turbine Reynolds numbers, even in steady-state. Thus, candidate airfoils should be tested in wind tunnels and in the field to acquire a steady-state database to support the more crucial unsteady studies which will eventually yield the best payoff. 


\section{CHAPTER 5. THE NEED FOR BASIC RESEARCH}

The preceding Chapters have dealt with specific aerodynamic study areas and high priority research needs for improvement in wind turbines. The Panel determined that the major work should be in unsteady aerodynamics and unsteady rotor inflow. The Panel recommends that in these areas the emphasis be on basic research, rather than on applied research.

\section{DEFINITION OF BASIC RESEARCH}

Basic research must be contrasted with applied research, which is strategic and is generally tied to a specific configuration. Applied research is also defined by its pertinence to a system rather than an entire range of systems. The basic research which is called for here must ultimately establish a technology base strong enough that design decisions can be made with confidence and not by trial and error as is of ten the case now. The Panel believes that very significant improvement in the performance and economics of wind turbines is possible given an adequate unsteady aerodynamics technology base.

\section{PRESENT CONFIGURATION STUDIES}

Configuration studies, designs, uses, and market bias, are the purview and necessary concern of industry. But, in the opinion of the Panel, present wind turbine configuration and design studies in both the government and industry programs have yielded about as much as they can. It is now time to turn to more basic studies. As in the example of 2-D steady vs. unsteady airfoil design, there is insufficient technology base and physical understanding to plan tests, to evaluate the results or to build adequate design tools. Design bias is common, and perhaps necessary, in configuration studies (i.e., HAWT vs. VAWT), especially in the absence of compelling physical and test evidence; however, design bias inhibits the process of understanding the underlying physics.

Furthermore, the industry hardware--the aerodynamic technology which is being applied--is not mature enough to be used to frame realistic cost and technology goals for a strategic (applied research) plan. The industry R\&D base is shallow, and has not pursued long-term unsteady aerodynamics goals. Organizations which have the depth to undertake the complex unsteady aerodynamics studies are now needed. Such groups might be universities, national laboratories, or established research arms of private industry. These R\&D organizations remain aloof from the configuration and market pressures, which are short term, and expend their effort on long term programs.

\section{THE NEED FOR UNSTEADY AERODYNAMICS EXPERIMENTAL STUDIES}

Wind turbine theoretical development has been seriously hampered by a lack of credible unsteady aerodynamics test data. Also, the generally-used HAWT prediction methods are heavily dependent on variations of steady-state momentum theory [13], and the VAWT theories depend on steady-state streamtube models, which largely ignore the unsteady effects [46]. 
For these reasons, the basic research program should include a strong experimental emphasis from the beginning. Tests need not necessarily be performed on wind turbine systems in the field, but under carefully controlled conditions which lend description to the unsteady events, and allow qualitative understanding. Turbines in the field are too complex for this process, but wind tunnels are not.

The initial advances in unsteady aerodynamics will undoubtedly come from the wind-tunnel tests; for example, terrain effects on siting can be studied this way. These tests, if done properly, can allow. unsteady events to be described and sensible empirical design tools put into practice even before they can be described mathematically. Historical examples are the NACA 4- and 5-digit series of airfoils, which were developed empirically and have proved to be very successful in aviation design. It is interesting to note that up to this point the most common airfoils in use on wind turbines are from these empirical families. Now, some of the promising new airfoils for wind turbine, the Wortmann FX84W series, are derived from more recent empirical studies [32].

Experimental studies can also help to define the proper directions for theoretical studies. An example here is the substantial wind-tunnel work done on dynamic stall prior to any attempts to describe it mathematically [9]. The unsteady aerodynamics capability to model the unsteady inflow is already well in hand with boundary-layer wind tunnels, which have been used extensively in the field of wind engineering, and will have much more application in aerometeorology.
The traditional scaling concerns of Reynolds number and Mach number, and wind-tunnel wall effects, are already used routinely in wind-tunnel modeling, even in unsteady flow. This is not to say the task is straightforward, but rather, that fruitful directions exist and are being researched [19].

Undoubtedly new scaling parameters will appear when the experimental studies begin to bear fruit. These will likely concern the scale parameters of the inflow fluctuations and parameters at the airfoil surface, such as reduced frequency. Field testing can also be productive, but only if the parameters are measured with enough care to extract the unsteady information.

\section{DIFFICULTY OF THE PROBLEM}

The nature of the basic research goals makes it difficult to estimate the time or cost needed for the recommended research study areas. Innovations and discovery of new approaches and solutions will modify any set of strategic goals or milestones adopted. The most that can be done is to define the higher payoff directions which are clear now, and will eventually bear fruit. We expect that these directions will also change in the future as knowledge is gained.

This course of action is not a short-term strategy, but a major, patient, continuing long term commitment. It depends on substantial directed resources and talent that carefully constructs theoretical programs coupled with strong experimental support, and is allowed to gain momentum over a space of years, probably decades. 


\section{CHAPTER 6. THE NEED FOR AEROMETEOROLOGY}

This Chapter describes the need for a new branch of aerodynamic study termed aerometeorology, which combines the unsteady aerodynamics of the wind turbine design community and the unsteady fluid mechanics of the meteorologists.

No one in the design community would argue with the quotation:' "With helicopters the rotor is the boss, and with wind turbines the wind is the boss [47]." The inflow and its content are the design drivers in productivity and reliability. The center for this inflow is currently the atmospheric physics community; specifically, it resides within the specialty called micrometeorology. However, in this country, at least, the communication between micrometeorologists and wind-turbine designers has been weak. Designers, knowing little about airfoil response in a fluctuating wind, have been unable to quantify their need for knowledge about turbulent gusts. Micrometeorologists, at the same time, have developed experimental and theoretical tools which might well prove adequate for gust description [48], but as a community have tended to focus on the rather different set of problems posed by weather prediction and population dispersion.

There are, of course, some exceptions; as an example, the wind-engineering group at Riso (Denmark) contains first-rate, mainstream micrometeorologists who have done innovative research on atmospheric turbulence as it bears on turbine design. As another example, the Panofsky-Dutton monograph Atmospheric Turbulence
[49] attempts with considerable success to relate the extensive body of micrometeorological data on turbulence structure to engineering applications. In general, however, these promising starts are the exception, and to date most of the effort in turbulence description within the U.S. wind engineering program has been done by those outside of the mainstream of micrometeorology.

A new field of study which combines micrometeorology and unsteady airfoil dynamics is therefore needed. Wind engineering is a relatively new discipline, and is a present experimental branch of technology which unites the expertise of meteorologists and aerodynamicists. The basic goal of aerometeorology is:

To define a disciplinary area of collaboration between the wind turbine engineering community and the micrometeorology community, both experimental and theoretical.

The engineering problem is depicted in Figure 27. If the theoretical development is not done, the experimental work will be unproductive, or at least severely limited in potential application and its ability to describe unsteady events. An example of a direction to start is the compendium of aircraft drag data compiled by Hoerner [50] in 1958 from all the available tests at the time in the literature. This text has proven valuable in the planning of theoretical aerodynamic studies and in design and analysis. Such a compilation could 


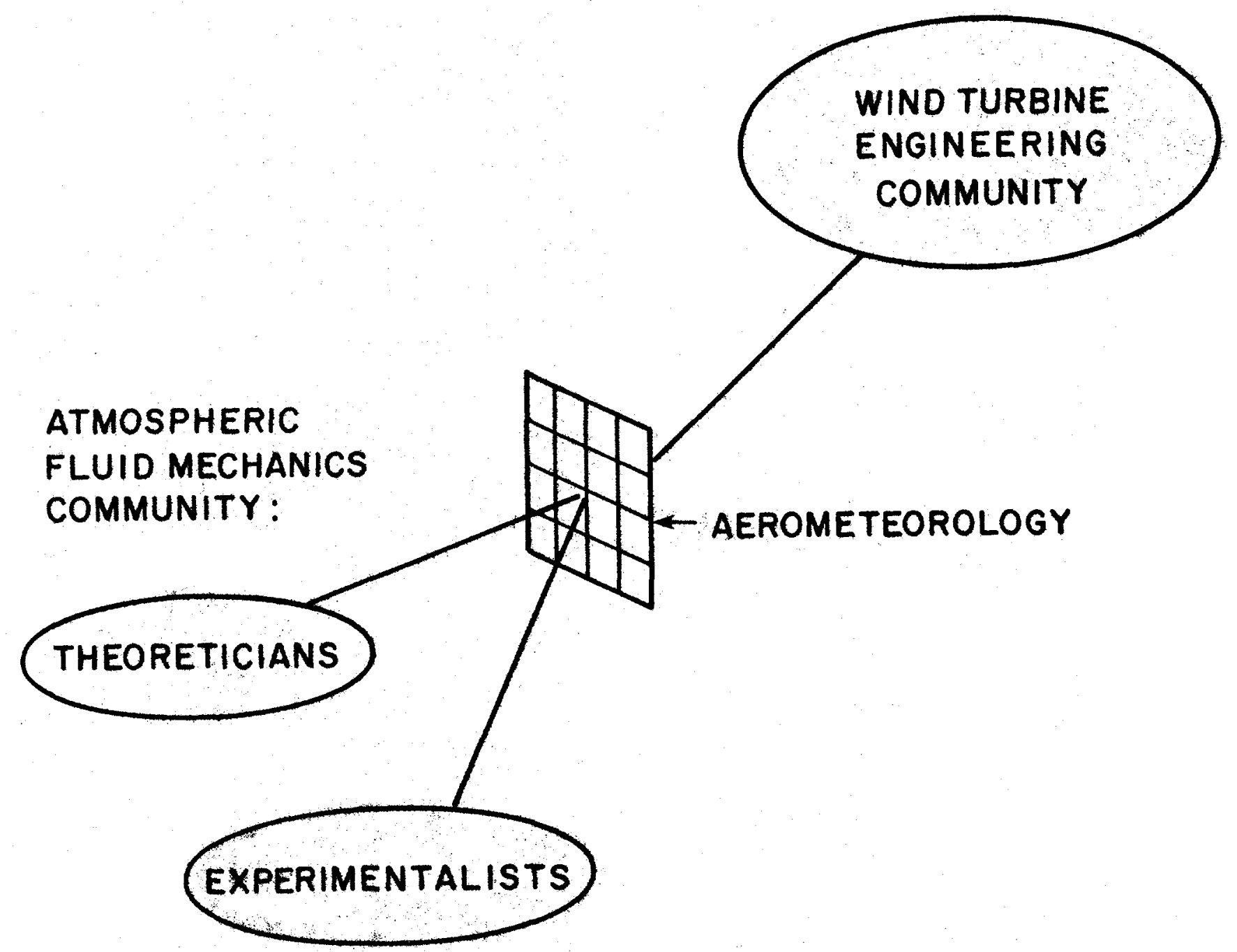


be started now in the wind turbine community for terrain effects, wake effects, gust loading, and yaw dynamics, to name just a few.

This Technical Assessment suggests the inflow model described in Chapter 3 for the first mathematical constraints of aerometeorology. This inflow model simply defines the linear fluctuation of an inflow front in a way that is understandable to both aerodynamicists and micrometeorologists. It could be the first step towards the interface between the two and the first construct of aerometeorology. The linear unsteady model could be used first in the studies of dynamic stall, and replaced by more complex models as understanding progresses.

In aerometeorology, the emphasis should be on representing realistic unsteady inflows to the aerodynamicists who will be assessing various design concepts. The initial burden may well be on the field measurement of fluctuations impinging on the test rotor, to represent them in enough detail to retrieve the key unsteady parameters. One approach has been suggested in Chapter 4 (Figure 22): the use of concentric rings of inflow sensors to "track" the nonuniform fronts through the rotor plane. A new type of field anemometer, which has the capability to remotely monitor both the mean and fluctuating components of the inflow, may also be necessary, and certainly complex real-time information retrieval and storage will be required. An analogy is the FAA use of towers with anemometers at airports to routinely measure turbulence significant to aircraft landing and takeoff. Another example, in ocean engineering, is the statistical assessment of ocean wave forces on a hull design [51]. Buoys are used at sea to measure and record wave motions in the frequency domain; model ship response is also measured for various wavelengths in a wave tank and a transfer function is constructed; the resulting forces are determined by superposition.

Two projects that could be undertaken immediately by aerometeorologists are the determination of effective rotor inflow length scales, and micrositing, or the representation of steady terrain interference inflow effects on distributions of mean inflow velocities.

Rotor Length Scale: Inflow fluctuations can be divided into two main groups: large-wavelength, low-frequency, representing potentially capturable energy, and short-wavelength, high-frequency, causing fluctuating airloads. What is the dividing line? Likely candidates on the rotor side are the diameter, the rate of change of angle of attack, and the duration of the fluctuation. On the atmospheric side are the longitudinal coherence, gust frequency, covariance, etc. The challenge clearly is to develop unsteady airfoil dynamics to the point where it can reveal the governing parameters on the rotor side, and to use the existing tools and techniques of micrometeorology to provide appropriate descriptors of the turbulent wind field on the rotor.

\section{Micrositing. Terrain and} Interference: The turbulence caused by complex terrain is complicated in that it varies in 
both space and time and interacts with the mean wind shear as well. The wakes of the machines themselves can also be troublesome, since they expose downstream units to additional turbulence and to a velocity deficit which can reduce power appreciably [26].

Progress toward a micrositing capability in complex terrain would seem also to require the combined skills of the micrometeorology and aerodynamics communities.

Boundary-layer wind tunnels have proven useful to simulating a variety of complex flows in the lower atmosphere, and should be immediately useful for studying wake effects. Micrometeorologists have made good progress recently in studying the modulation of surface-layer turbulence by complex terrain; that work, carried out in connection with turbulent dispersion programs, could provide a basis for a parallel effort here. Again, however, we feel that longterm progress in this topic requires that aerometeorologists establish a sensible theoretical framework which will allow these studies to proceed systematically and in concert with related studies in mircometeorology and in aerodynamics. 


\section{CHAPTER 7. APPLICATIONS STUDIES}

There are a number of other topics of wind turbine engineering study in which aerodynamics plays a major role, but which do not fall into the category of basic research. These studies, although of lower priority, are important nonetheless:

\author{
- Noise \\ o Rotor Size, Productivity and \\ Economy of Scale \\ - Variable Speed Rotors \\ - Advanced Concepts \\ o New Descriptors
}

\section{NOISE}

Rotor noise, especially blade tip noise at high speed, will be an important siting criterion. The more efficient high tip speed ratio designs will likely be needed for the low-to-moderate wind resource areas which are marginal for present design approaches (see Appendix 2). In rotorcraft, perceived noise is correlated with tip speed, disc loading, and range to the rotor; see Figures 28-30 [52]. Present stall-controlled wind turbines employ low tip speeds in part to reduce perceived noise. However, it is clear that benefits could be obtained in the larger rotor sizes by increasing tip speed.

Perceived noise correlated with tip speed for helicopters is plotted in Figure 28 [52]. The Mach number dependency of generated noise can be seen. The table below gives the design rotor tip speeds for some present wind turbines. It can be seen that the wind turbine tip speeds are much lower than helicopter tip speeds.

Figure 29 [52] compares aircraft and present wind turbine noise levels [53] to typical freeway and urban street noise, as a function of distance to the source. The rapid attenuation of noise with distance can be seen. The last diagram, Figure 30, shows noise levels of aircraft and wind turbines at a range of 400 feet vs. the disc loading (the thrust force divided by the rotor area). It can be seen that the disc loading range of wind turbines is larger than for helicopter rotors and propellers, since the wind turbines operate in a much wider variety of thrusting conditions. The wind

WIND TURBINE DESIGN TIP SPEEDS

\begin{tabular}{lcc}
\multicolumn{1}{c}{ Turbine } & Diameter & Design Tip Speed \\
\hline & $38 \mathrm{~m}$ & $264 \mathrm{ft} . / \mathrm{sec}$. \\
MOD-0 & $61 \mathrm{~m}$ & 366 (lowered to 241) \\
MOD-1 & $91 \mathrm{~m}$ & 275 \\
MOD-2 & $79 \mathrm{~m}$ & 340 \\
WTS 4 & $98 \mathrm{~m}$ & 293 \\
MOD-5 & $15 \mathrm{~m}$ & 150 \\
Typical Danish & $25 \mathrm{~m}$ & 310 \\
Petten (Dutch) & $41 \mathrm{~m}$ & 215 \\
Nibe (Danish) & $100 \mathrm{~m}$ & 270 \\
Growian (German) & & \\
\hline
\end{tabular}


FIGURE 28. ROTOR NOISE: EFFECT OF TIP SPEED

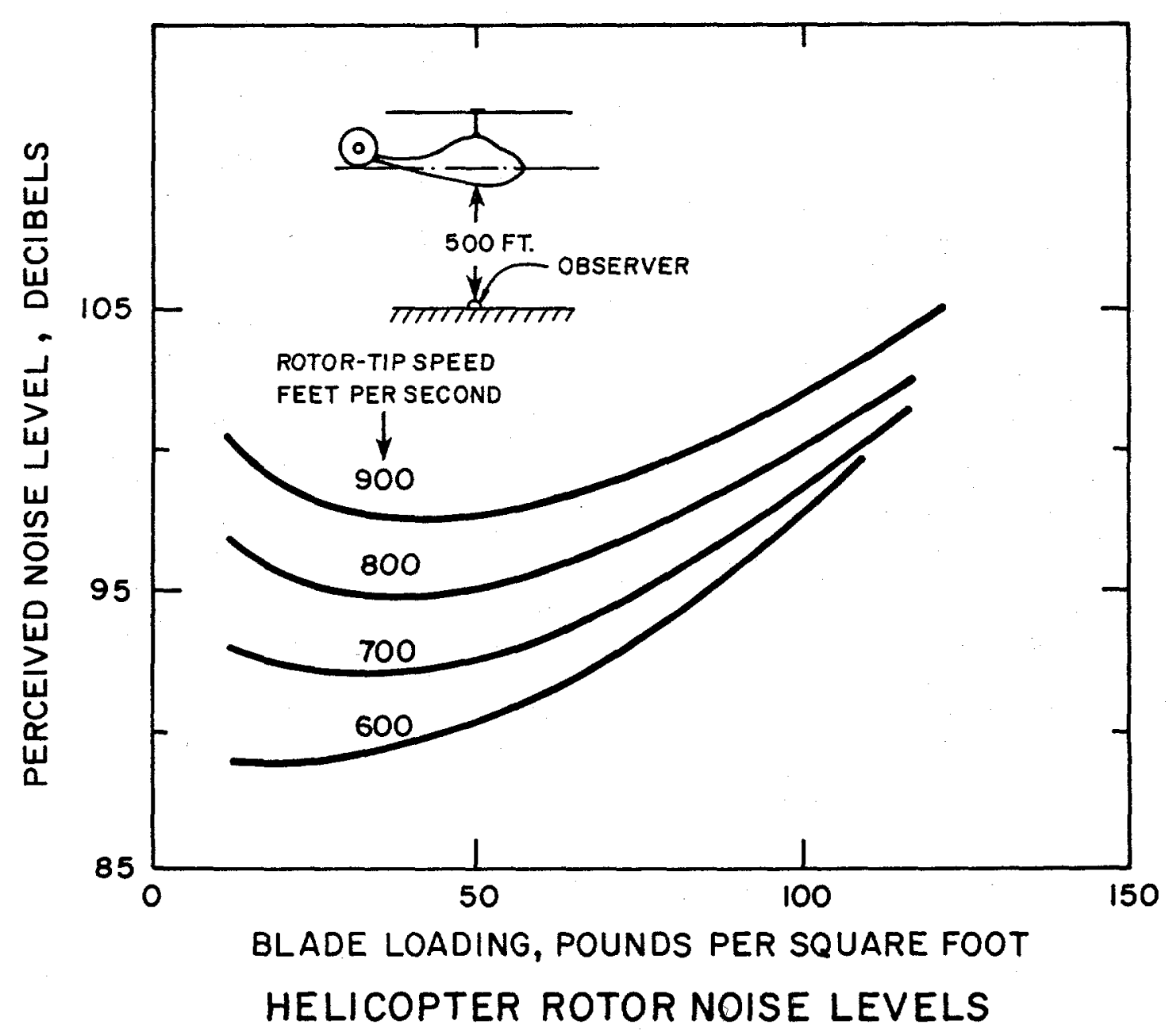


FIGURE 29. ROTOR NOISE: TYPICAL COMPARISON $[52,53]$

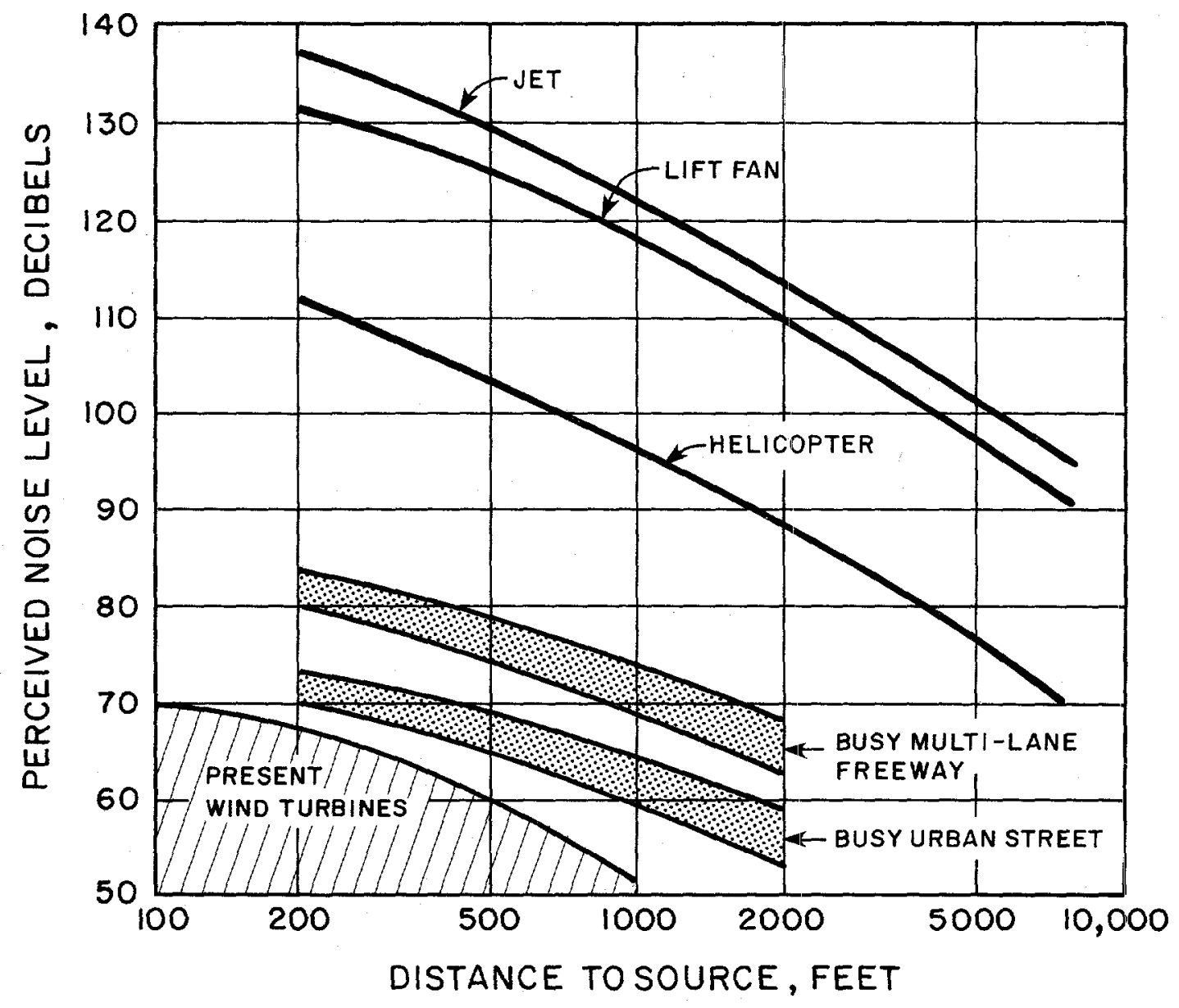


FIGURE 30. ROTOR NOISE: EFFECT OF DISC LOADING [52, 53]

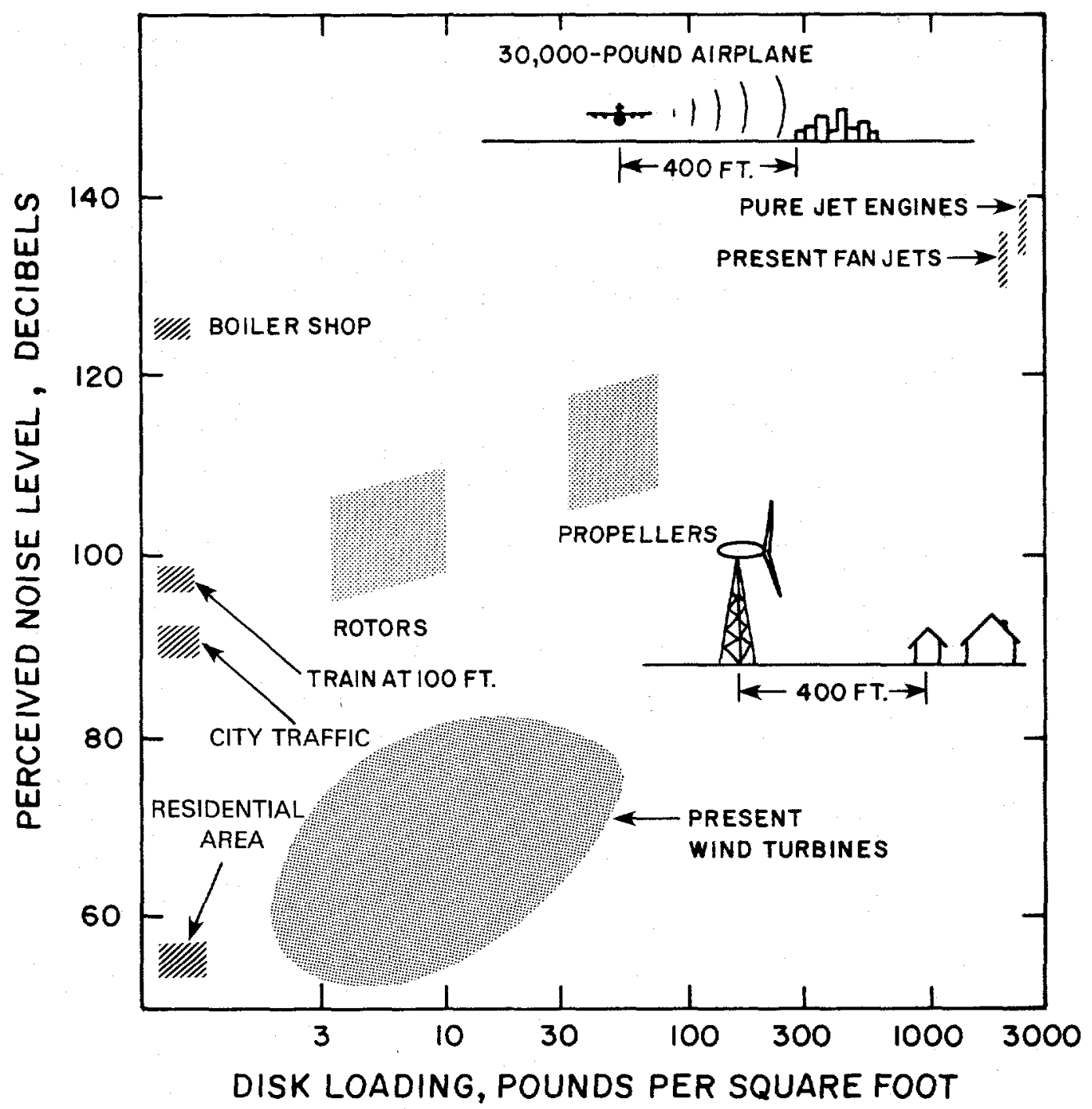

EFFECT OF DISC LOADING ON PERCEIVED NOISE LEVELS 
turbine noise at the higher disc loadings appears to be largely masked by the ambient wind noise itself.

We caution that these wind turbine noise data are only approximate and the results remain inconclusive. More acoustic tests are necessary before the noise boundaries can be refined. Tip noise is one of the first measurable environmental effects of wind turbines, and prediction analysis must be developed early in order to satisfy siting constraints.

The aeroacoustic prediction codes have been extensively developed for the very general rotorcraft cases. The capability to predict aerodynamic noise is, therefore, well in hand provided the unsteady airloads can be determined. For wind turbines, the technology need is to provide these unsteady loads to the prediction programs. Additionally, new noise field (pattern) descriptors, and "quality" parameters will likely be needed, since perceived noise has always been a partly subjective study (e.g., pulsed vs. continuous) [54].

\section{ROTOR SIZE, PRODUCTIVITY, AND ECONOMY OF SCALE}

An aerodynamic economy of scale is possible with larger wind turbine rotors. Large turbines are more land efficient (see Figure 31 [54]), are higher in the atmospheric boundary layer thus are usually subject to higher winds, and have larger blade chords leading to potentially more desirable Reynolds numbers and a higher lift/drag ratio. As a result, specific output per swept area can be expected to increase with rotor diameter. This trend is shown in Figure 32, which presents the calculations compiled by le Gourieres [55] and a number of other turbines. The theoretical annual output was estimated by assuming a Weibull wind speed distribution with an annual average of $6 \mathrm{~m} . / \mathrm{sec}$. (13.4 MPH) 10 meter height and using the $1 / 7$ power law for hub height scaling. The curve shows estimated $\mathrm{KWh} /$ square foot of rotor area/year vs. rotor diameter for wind turbines from 6 feet (1.8 meters) to 329 feet (100 meters) in diameter; these are also listed in Figure 33.

At the lower diameters, the bulk of the machines follow a clear economy of scale. However, this is not a clear cut trend as evidenced by the outlying turbines.

Also, the curve is misleading, since the ability to produce energy increases as the square of the diameter. Therefore, a truer comparison is a plot of specific output vs. rotor area, Figure 34. By looking at this graph and considering the outliers, it is clear that the economy of scale argument is not simple. If there were no influence of size on economy, this plot would show a horizontal line.

Figure 35 is a pictorial representation of the aerodynamic effects which change the straight line ideal, and the direction of their expected effect, for large wind turbine rotors ( $200 \mathrm{ft}$. [61 meters] diameter and larger). The Reynolds number, wind shear, and wind turbulence effects are shown schematically. Another beneficial effect is the lower rotor solidity likely with large rotors, and the resulting lower disk loading necessary to produce the same torque, 

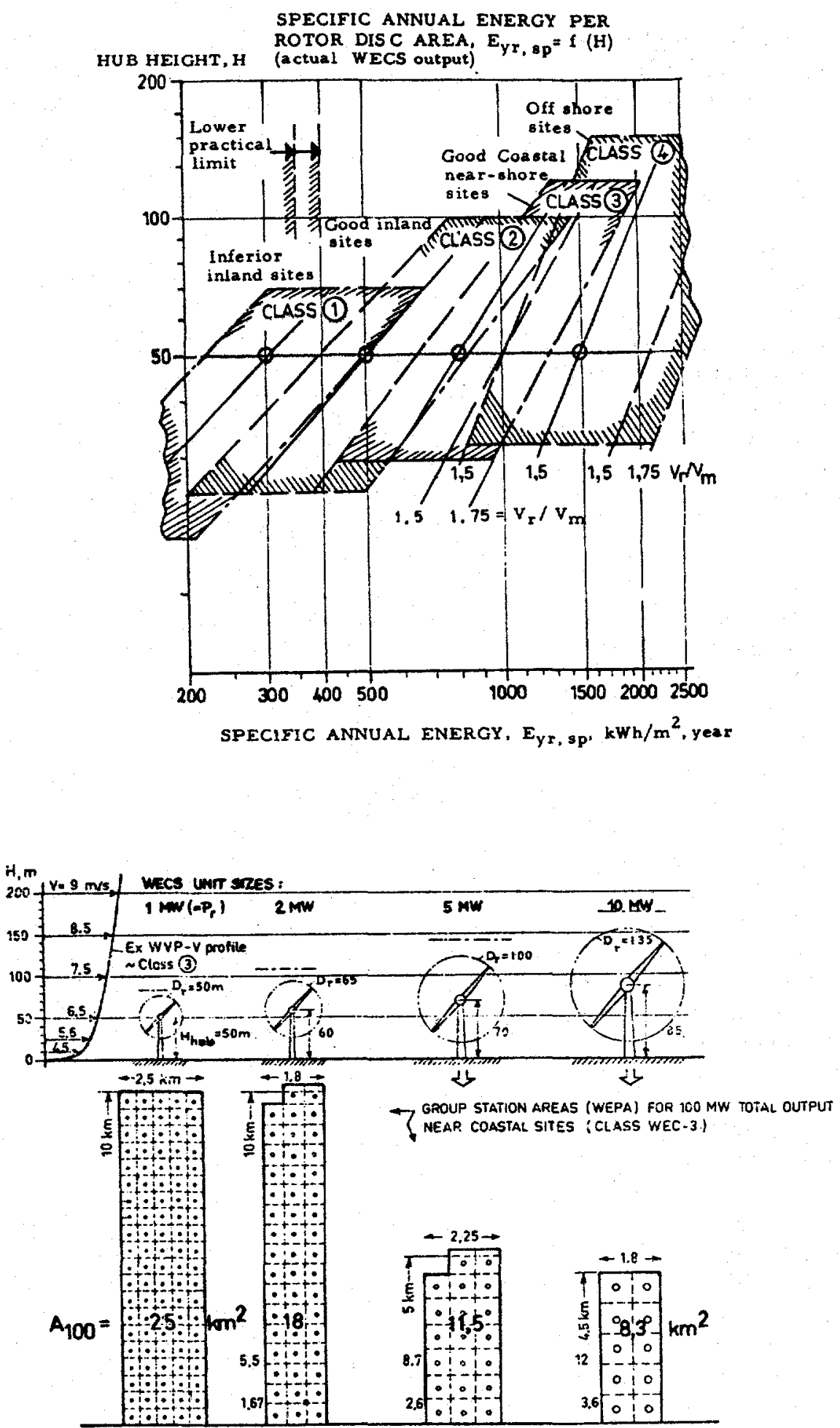

Effect of WECS Unit Size, $P=1-10 \mathrm{MW}$, on Group Station Land Area. Preliminary Trend Estimate for Example - $100 \mathrm{MW}$ Group Station 
FIGURE 32. SPECIFIC PRODUCTIVITY VS. ROTOR DIAMETER

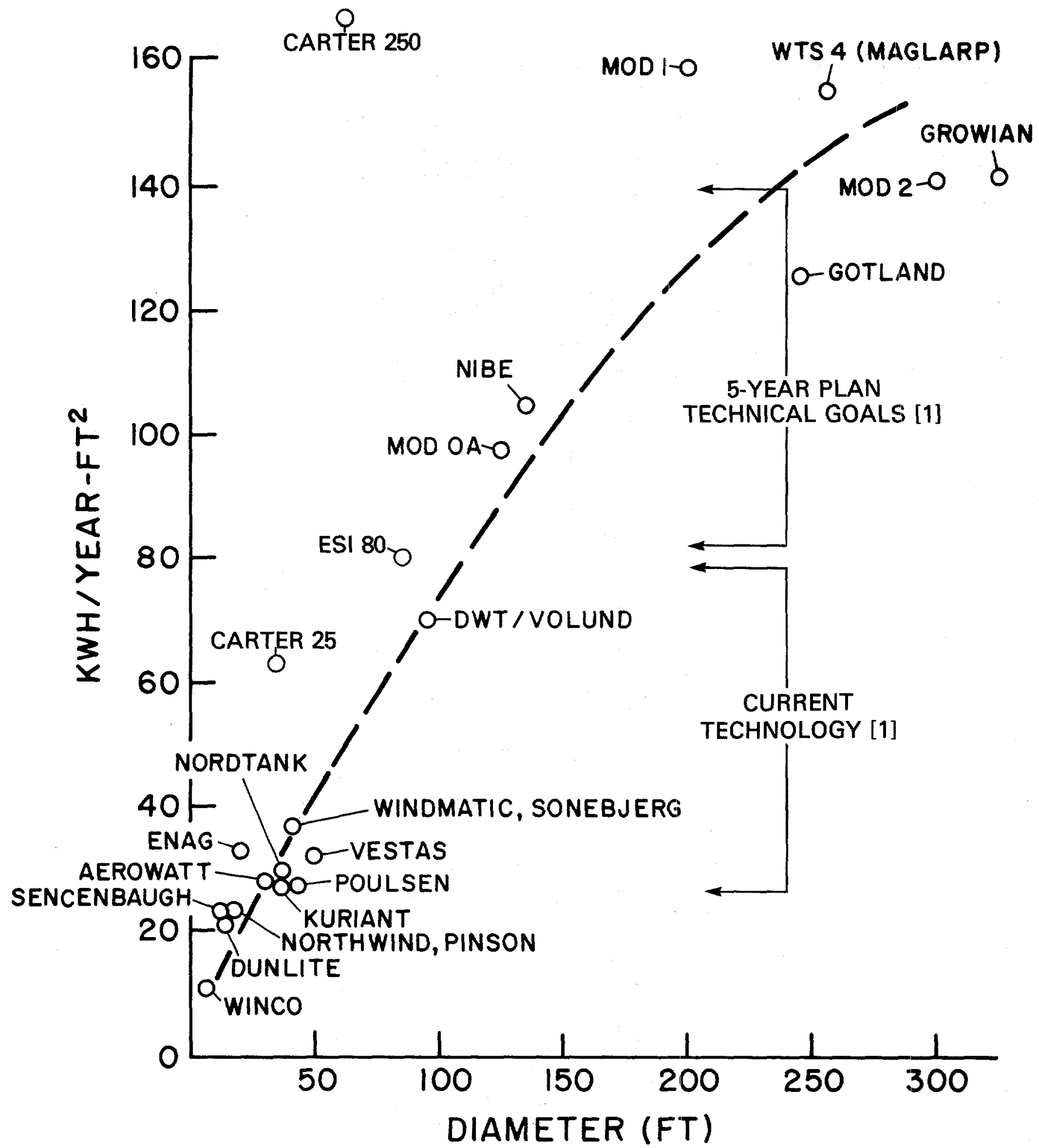


FIGURE 33. WIND TURBINE ENERGY PRODUCTIVITY (ESTIMATED) [55]

\begin{tabular}{|c|c|c|c|c|c|c|}
\hline \multicolumn{3}{|c|}{ DIAMETER } & \multicolumn{2}{|c|}{ PRODUCTIVITY } & AREA & SPECIFIC OUTPUT \\
\hline TURBINE & $m$ & ft. & KW & KWh / yr. & ft. & $\mathrm{KWh} / \mathrm{ft}-\mathrm{yr}$ \\
\hline WINCO & 1.8 & 6 & 0.2 & 300 & 28 & 10.7 \\
\hline SENCENBAUGH & 3.6 & 11.8 & 1.0 & 2,500 & 109 & 22.9 \\
\hline DUNLITE & 4.1 & 13.5 & 2 & 3,000 & 143 & 21 \\
\hline NORTH WIND & 5 & 16.4 & 2 & 5,000 & 211 & 23.7 \\
\hline PINSON & 5 & 16.4 & 5 & 5,000 & 211 & 23.7 \\
\hline AEROWATT & 9.2 & 30.2 & 4.1 & 20,000 & 716 & 27.9 \\
\hline ENAG & 6 & 19.7 & 6 & 10,000 & 305 & 32.8 \\
\hline CARTER 25 & 10 & 33 & 25 & 55,000 & 855 & 64 \\
\hline KURIANT & 10.9 & 36 & 15 & 28,000 & 1018 & 27.5 \\
\hline NORDTANK & 11 & 36 & $7.5 / 22$ & 30,000 & 1018 & 29.5 \\
\hline WINDMATIC & 12 & 39.4 & 30 & 45,000 & 1219 & 36.9 \\
\hline SONEBJERG & 12 & 39.4 & 45 & 45,000 & 1219 & 36.9 \\
\hline U. POULSEN & 13 & 43 & $5.5 / 30$ & 40,000 & 1452 & 27.5 \\
\hline VESTAS & 15 & 49 & $5 / 45$ & 60,000 & 1886 & 31.8 \\
\hline CARTER 250 & 20 & 65 & 250 & 550,000 & 3318 & 166 \\
\hline ESI 80 & 24.4 & 80 & 260 & 400,000 & 5025 & 79.6 \\
\hline VOLUND/DWT & 29 & 95 & 260 & 500,000 & 7088 & 70.5 \\
\hline MOD-OA & 38 & 125 & 200 & $1,200,000$ & 12272 & 97.8 \\
\hline NIBE & 41 & 135 & 630 & $1,500,000$ & 14314 & 104.8 \\
\hline MOD1 & 61 & 200 & 2000 & $5,000,000$ & 31416 & 159 \\
\hline GOTLAND & 75 & 246 & 2000 & $6,000,000$ & 47529 & 126.2 \\
\hline WTS 4 & 78 & 256 & 3000 & $8,000,000$ & 51472 & 155.4 \\
\hline MOD2 & 91.5 & 300 & 2500 & $10,000,000$ & 70686 & 141.2 \\
\hline GROWIAN & 100.4 & 329 & 3000 & $12,000,000$ & 85012 & 141.2 \\
\hline
\end{tabular}


FIGURE 34. SPECIFIC PRODUCTIVITY VS. ROTOR AREA

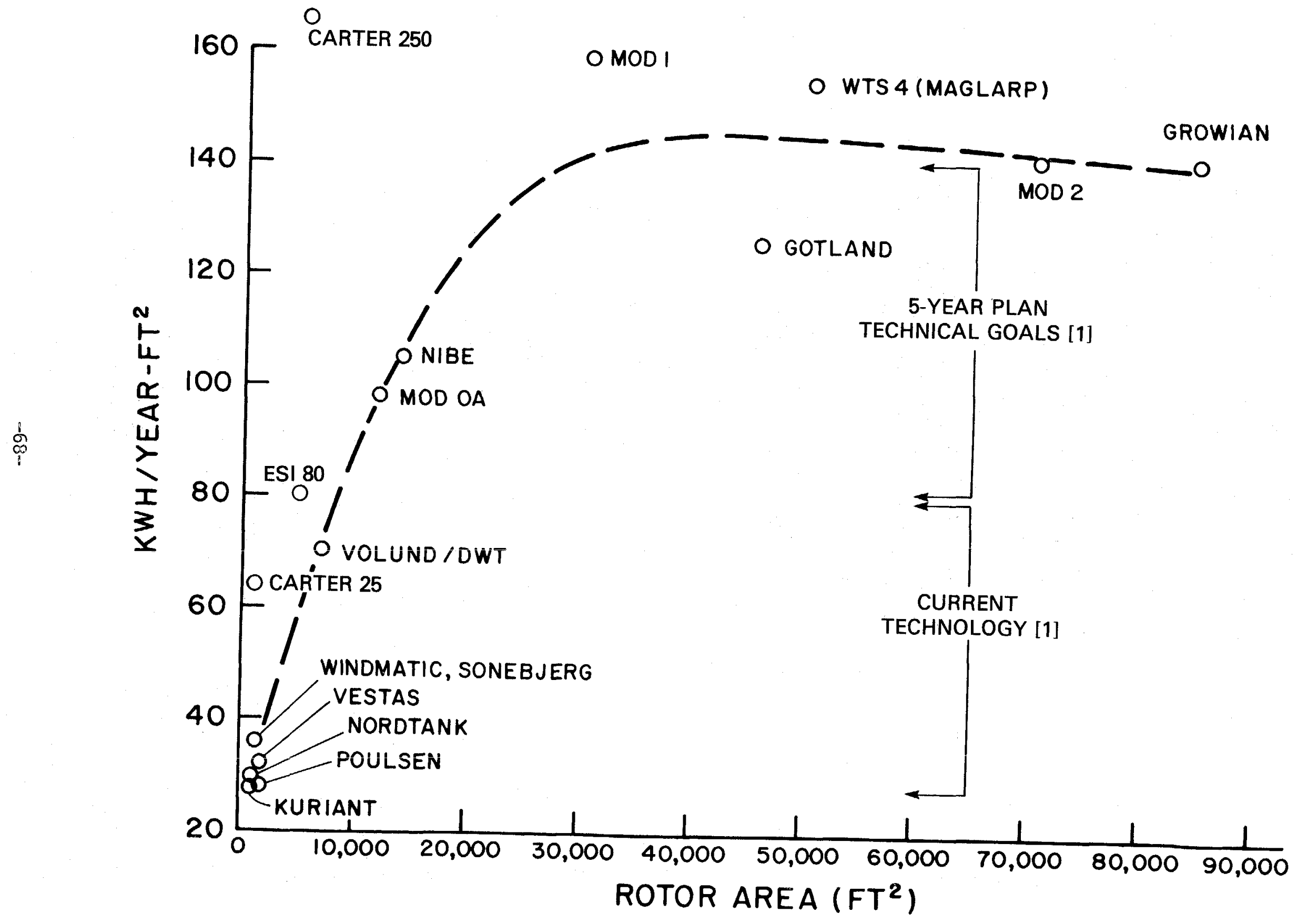


FIGURE 35. ECONOMY OF SCALE DRIVERS FOR LARGE WIND TURBINE ROTORS

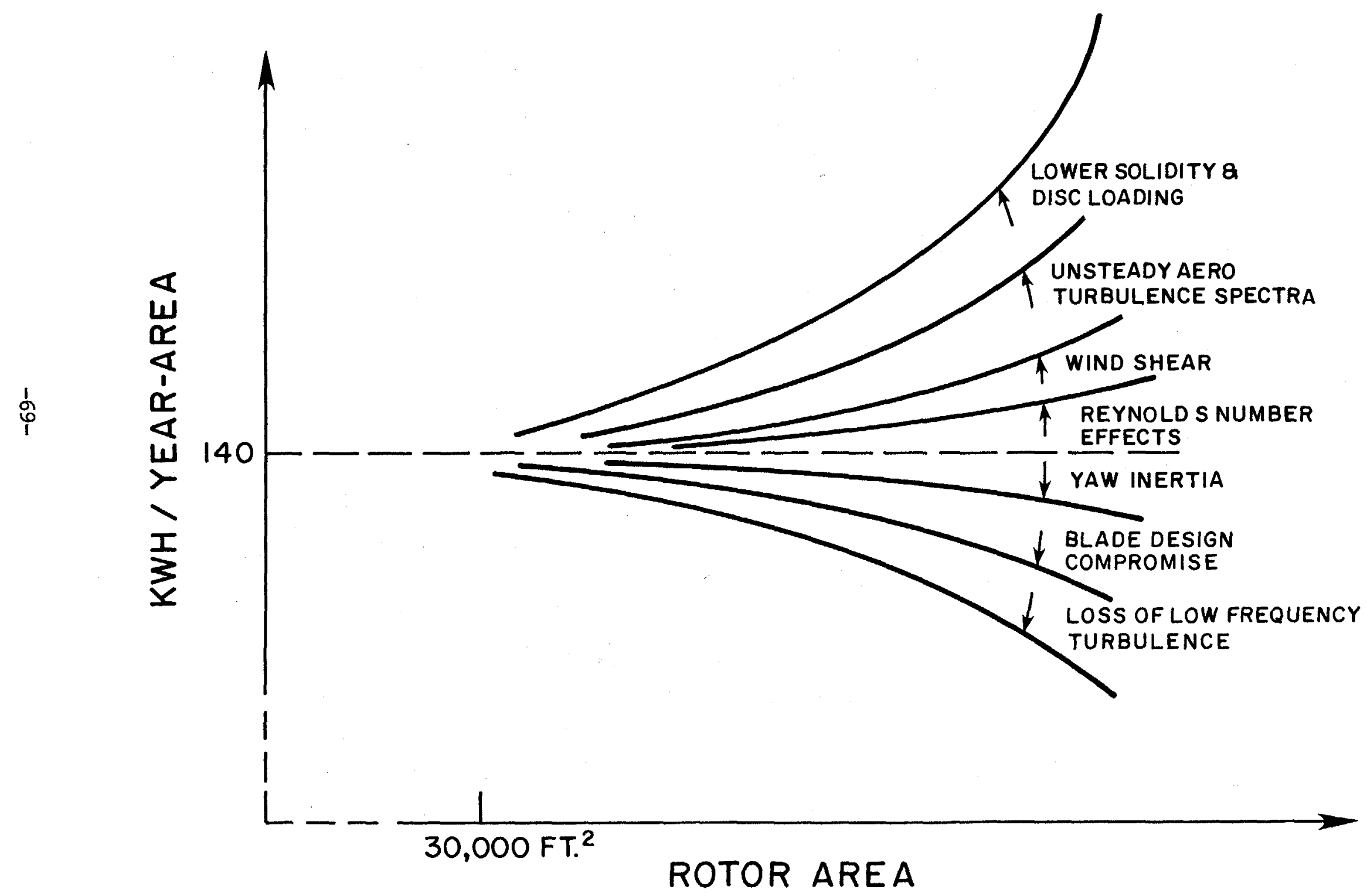


called the "CT/sigma effect".

Stated another way, a low-solidity, high tip speed ratio wind turbine has a higher usable power coefficient (efficiency) and operates at lower average angles of attack.

Effects which could degrade the economy of large scale performance are higher yaw inertia of $f$ tracking, blade aerodynamic compromise due to structural requirements, and the loss of low-frequency "capturable energy" wind fluctuations. Clearly, more needs to be known about the unsteady performance of the rotors and the representation of the unsteady inflow, before a definitive conclusion can be made about aerodynamic economy of scale of wind turbines.

\section{VARIABLE SPEED ROTORS}

A variable speed rotor which operates at constant tip speed ratio is attractive. Its most significant advantage is that the rotor operates at constant power coefficient when it is at constant tip speed ratio, and this can be designed to be the maximum power coefficient (rotor efficiency) for the rising power range (region 2), and steadily lower power coefficients for the load control range (region 3; see Fig. 17). Additionally, the variable speed rotor uses a constant pitch angle to maintain a constant power coefficient, so no pitch change is required.

Another advantage of a constant tip-speed ratio rotor, but one which is not so easily quantified, is the equilibrium of operation.

Simply stated, the aerodynamic and inertial rotor blade loads are in balance for all inflow wind speeds. This can be seen by referring to Figure 26. As wind speed increases, RPM increases

proportionally; aerodynamic loads including rotor thrust and torque increase as the square of wind speed, and the offsetting inertial centrifugal forces balance since they increase as the square of RPM as well. Therefore, the average coning angle of a constant tip-speed ratio turbine is constant. It is not clear what performance improvements this could eventually bring, but potentially beneficial aspects are:

1. The variable speed, constant tip speed ratio operation is a true "point design", with all fluctuations occurring around the same, specified operational characteristic.

2. The wake state and load equilibrium are constant.

3. Aerodynamic equilibrium is maintained; therefore, any beneficial unsteady aerodynamic condition which is specified, such as a standing tip vortex, will be maintained.

4. By allowing a utility grid-connected turbine to operate at variable speed, the rotor is effectively de-coupled from the load, and is therefore less vulnerable to power grid discontinuities, and has beneficial aerodynamic damping to variations in the rotor inflow.

As rotor size increases these arguments continue to hold identically; therefore, tip speed ratio is a powerful scaling parameter. To a first approximation, the tip speed ratio is just proportional to 
the angle of attack of the turbine: decreasing the tip speed ratio uniformly increases angle of attack [56], and vice-versa; this also can be seen by referring to Figure 26 . The size limitation of variable speed turbines is apparently structural, since gravity loads do not scale or stay in equilibrium as do aerodynamic and inertial loads [57].

Therefore, we perceive a need for the study of constant tip speed ratio turbines, particularly under unsteady conditions.

\section{ADVANCED CONCEPTS}

There is potential for large improvement in specific output of wind turbine rotors by using advanced aerodynamic concepts. An example is the shrouded turbine, Figure 36. Possible aerodynamic benefits which could be achieved are: increased mass flow thus power augmentation; lower sensitivity to yaw off-tracking; lower fluctuations in torque due to damping of inflow; and higher turbine speed and efficiency. Whether these benefits would outweigh the disadvantages of complexity, cost and weight remains a question for design study. This study cannot be effectively accomplished until the unsteady inflow has been sufficiently described, but the other design tools are well in hand. For example, a duct lift coefficient of 3.0 , which is achievable with standard leading-edge devices such as triple-slotted or Fowler flaps, will theoretically give a power flux increase by a factor of five (see Figures 37 [15] and 38 [58]).

The ducted turbine is just one example of a family of advanced concepts, all of which have the potential to exceed the Betz limit on power coefficient (0.5926) based on rotor swept area; this is generally held to be the maximum for conventional HAWT rotors [15, 55]. The physical interpretation of the Betz limit for VAWT is not clear, and this is complicated by the added unsteadiness of the affected streamtube and turbine wake.

Another example is the tip vane, Figure 39 [59]. Power coefficients based on rotor swept area above 2.0 have been demonstrated in wind-tunnel tests. The aerodynamic mechanism appears to be a standing vortex ring produced by winglets at the tips, so positioned that they induce a convecting pair of tip vortices in the wake flow which interfere constructively to produce a weak aerodynamic equivalent of a solid duct. Increased mass flow then results as long as the geometry is maintained. The wake state and geometry must be preserved by operating at constant tip speed ratio, and the inflow must be steady. Substantial augmentation has not yet been achieved in field tests [60], but we believe it is likely that it eventually will be demonstrated. Again the research need is for proper unsteady flow description and testing.

Other advanced concepts which might obtain higher performance than conventional systems are variable geometry rotors, including variable twist, variable diameter, and variable chord.

The potential improvements offered by these concepts are tempting to exploit, but much effort may be wasted if realistic, unsteady conditions are not considered at the very outset of a 
FIGURE 36. SHROUDED WIND TURBINE ROTOR

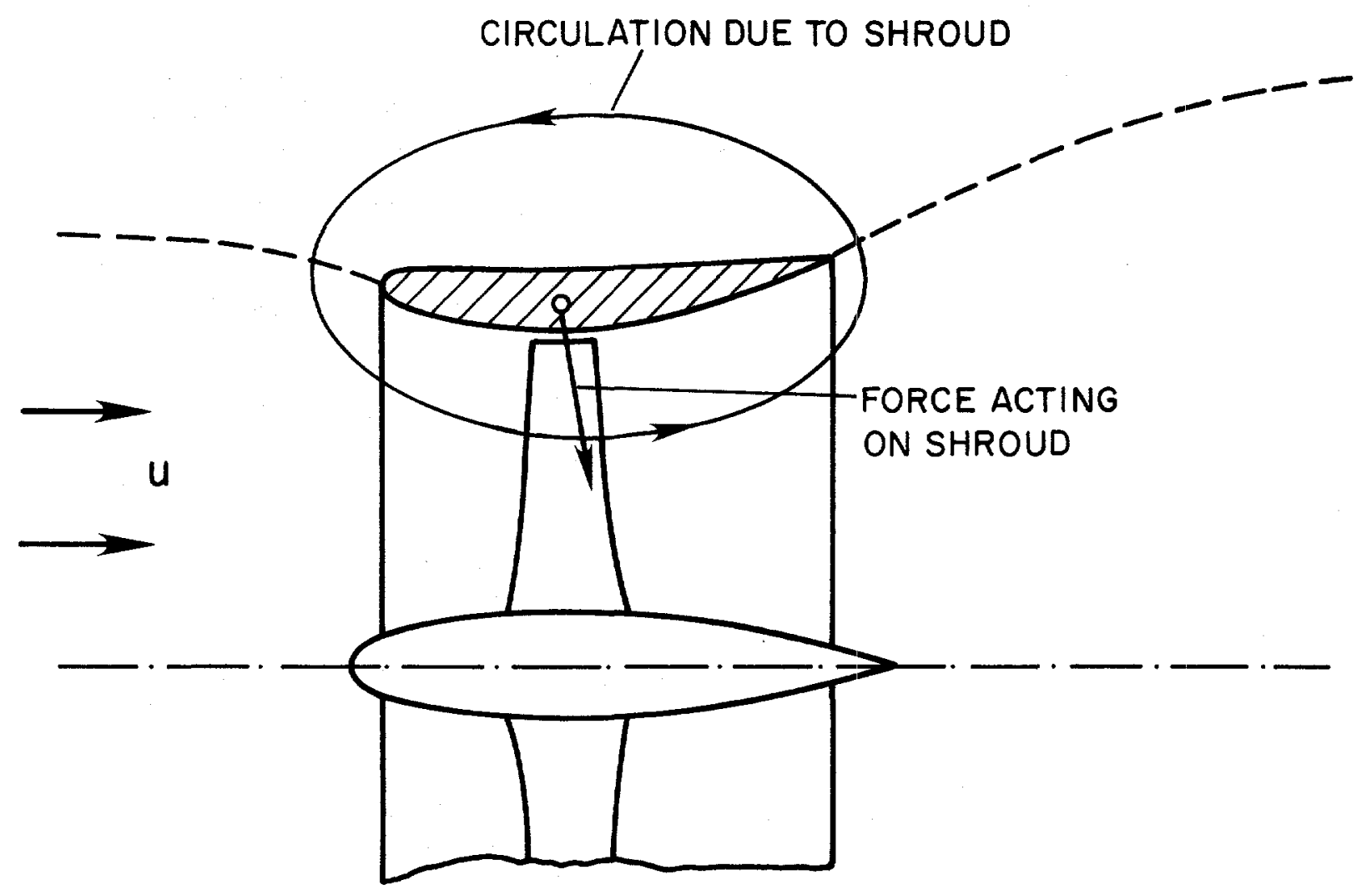




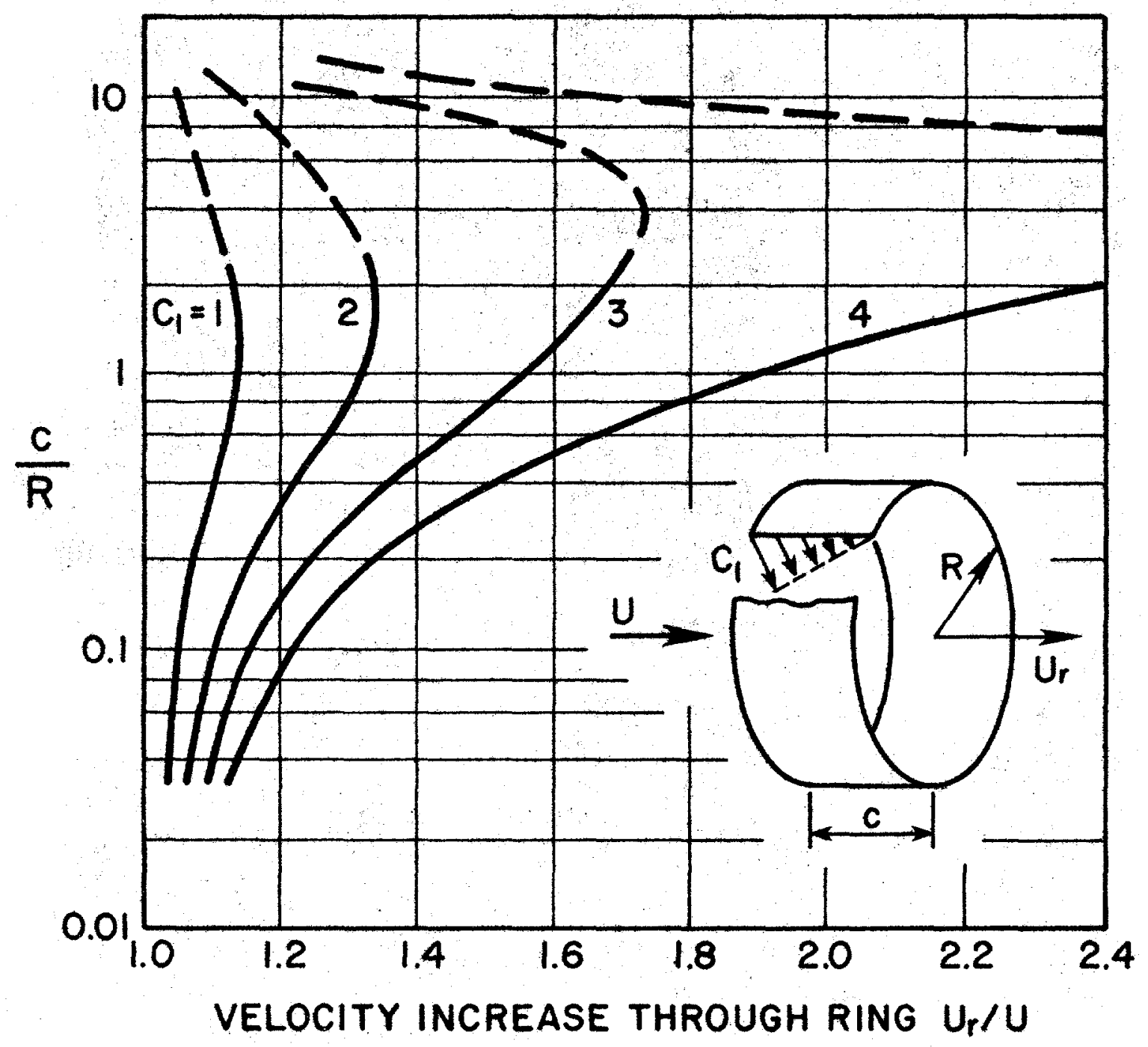




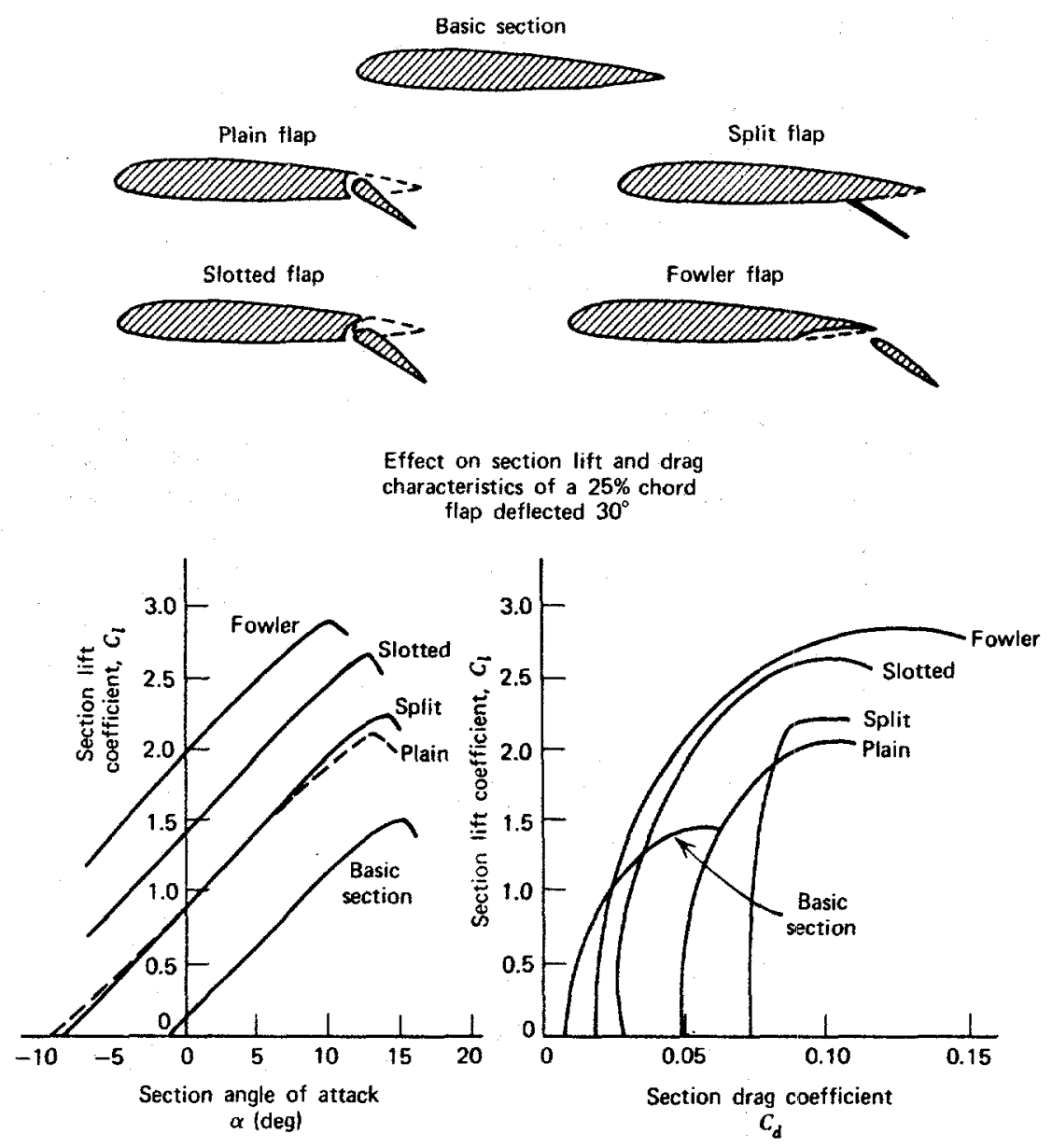


FIGURE 39. TIP VANE ROTOR
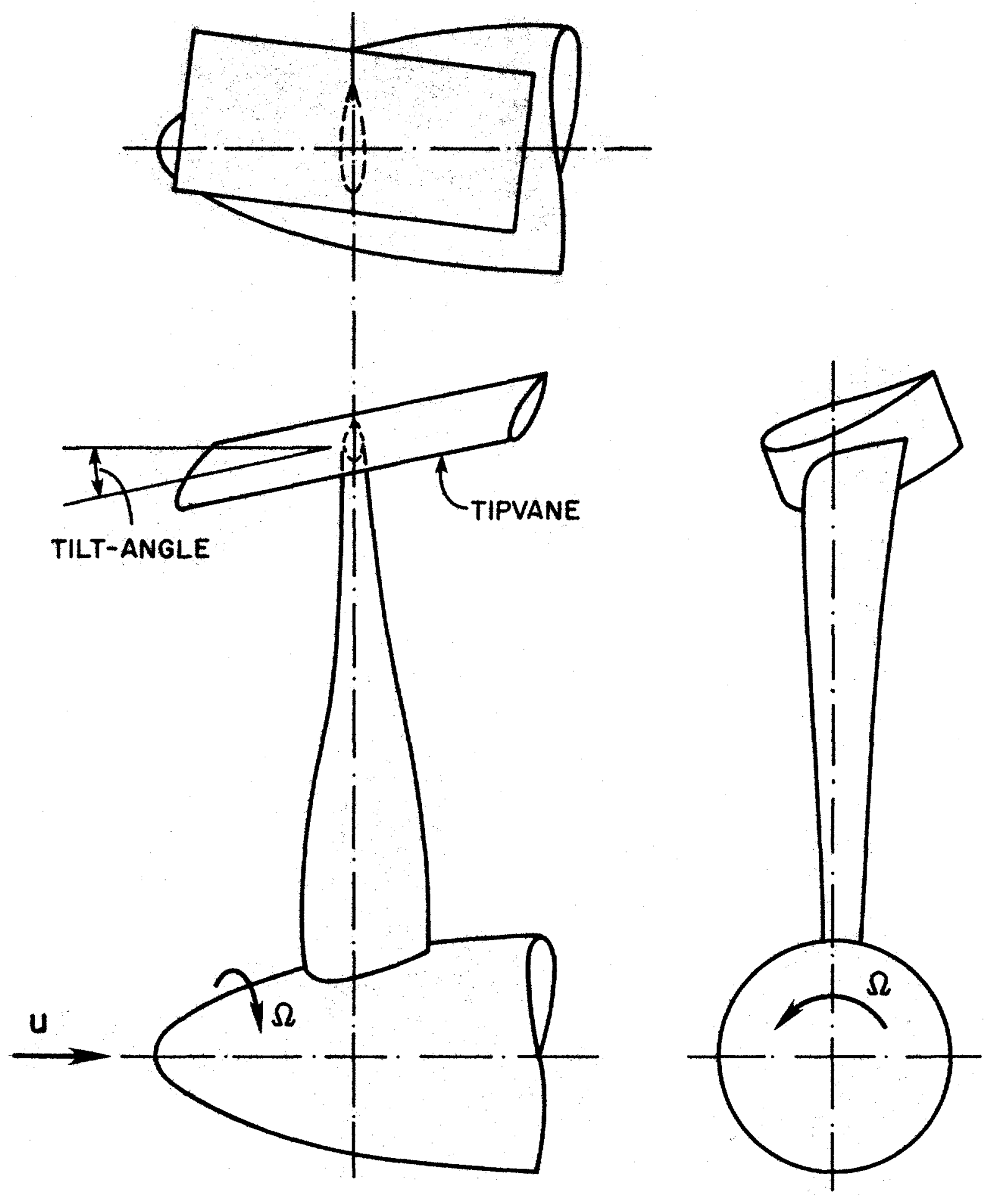
development program. The technology base is very embryonic for these rotors as contrasted with the more conventional HAWT rotors or even the existing VAWT rotors.

\section{NEW DESCRIPTORS}

Non-dimensional parameters have been valuable in the design development of aircraft and rotorcraft. The most valuable use of non-dimensional aerodynamic quantities has been in the scaling and similarity studies necessary for small-scale controlled field and wind tunnel testing. However, these parameters are also useful in comparing various design concepts for performance and reliability.

Aerodynamic research has tended to use these traditional non-dimensional quantities to frame its planning and judge its own progress. Some are general, and are presently used for wind turbines as well (e.g., Reynolds number, Mach number). Wind turbine studies have also generated some quantities specific to wind turbines, for example, thrust coefficient, power coefficient, and tip speed ratio. (Refer to Appendix 2 for the difference between the wind turbine and helicopter definitions.)

Additional descriptors need to be adopted which are relevant to wind turbines, both in the aerodynamics and in the system analysis. Some possibilities are: a plant/capacity factor of the rotor that would be useful to compare with other competing power plants [61]; a nondimensional installed torque vs. produced torque, to be used for the comparison of different turbines with varying installed power-to-rotor size ratios; and a design rotor "lift coefficient" that includes the expected moments of inflow fluctuation around the equilibrium condition, to judge the percentage of off-design availability.

We believe that the search for these should properly begin in the examination of test data, where various trials can be made to discover generality, and in basic studies of unsteady airfoil behavior and unsteady rotor inflow. An example is the reduced frequency, which is one of the driving (but maybe not the most significant) conditions for dynamic stall. 
In. 


\section{CHAPTER 8. RECOMMENDED DOE WIND TURBINE UNSTEADY AERODYNAMICS R\&D PROGRAM}

\section{PRESENT FEDERAL WIND ENERGY PROGRAM}

This Chapter outlines aspects of a national R\&D program for implementing the wind turbine unsteady aerodynamics research needs put forth in this Report. This effort must and can occur within the present framework of the current DOE wind energy program, which is described below.

A description of the present federal wind energy program is quoted below from the Five Year Wind Energy Program Plan [1]. The three major parts to the present DOE wind energy program are (1) sponsor basic research, (2) conduct research on advanced components and systems, and (3) transfer the research results to industry:

"Federal policy on energy research and development (R\&D) is to sponsor activities that are unlikely to be carried out by private industry because of the technical and financial risks involved. Accordingly, the goal of the Federal Wind Energy Program is to conduct research to establish a technology base and to support industry in confirming the viability of wind energy as an energy supply alternative. This research will complement and support private sector efforts to develop wind systems that are safe, reliable, and cost-effective.

The Federal Wind Energy Program's R\&D strategy has three main elements. First, the program will sponsor basic research on the science of wind turbine dynamics. This research must be conducted to understand the random nature of the wind resource itself, its complex interaction with the wind turbine, and the effects of this interaction on performance and reliability. Second, advanced components and systems research will be conducted. This research will establish the technology for advanced components such as high-performance airfoils designed specifically for wind turbine operation. The program will verify research results through testing on DOE test beds and through cooperative field test programs on commercial machines. Finally, the program will transfer the research results to industry for use in developing cost-effective wind turbines.

Successful implementation of this R\&D strategy requires an effective partnership between government and industry. The federal role in the development of wind energy technology must complement and support industry research and development. Federal $R \& D$ activities are aimed at supporting the general advancement of the technology by providing the scientific knowledge that industry can then apply in the development of improved machines. To ensure the efficient use of public resources, the federal government focuses its efforts on activities that industry is not expected to undertake on its own because of the cost, risks, or development time involved. In general, the program takes primary responsibility for working with industry to determine long-term research needs, sponsoring the research needed to address those priorities, and disseminating research results. The federal program then relies upon industry to apply the technology base in 
order to improve the cost, performance, and lifetime of future wind machines [1]."

The current DOE plan thus places increased emphasis on an improved understanding of the basic physical phenomena involved in converting the wind to useful energy. The (draft) November 1985 Revised Comprehensive Program Management Plan [62] goes further in this regard to establish objectives in specific areas in which technical advances are required:

- "Improve the understanding of fundamental sources of wind variability--local windflow variability and shear, and turbineto-turbine interactions.

- "Increase basic understanding of the interactions between wind input and structural response and the resulting effects on performance and loads.

o "Investigate, through generic, proof-of-concept activities, the potential for improved performance using advanced components and subsystems.

- "Develop an advanced multi-megawatt wind turbine [62]."

\section{ROLE OF PRESENT AERODYNAMICS TECHNICAL ASSESSMENT}

The wind turbine aerodynamics $R \& D$ programs recommended by the Panel in this Technical Assessment address the first two objectives stated above. The specific subproblems that properly delineate the two objectives are the research needs recommended by the Panel in Chapter 4:

\section{Unsteady Aerodynamics}

Dynamic Stall Understanding--

Develop an understanding of the 2-D and 3-D hysteresis effects of dynamic stall.

Testing Methodology for Unsteady Flow--

Stimulate complex unsteady flows in wind tunnel and total system (field) tests in order to exploit dynamic stall effects.

Airfoil Development Studies for Unsteady Flow--

Initiate a design process which will yield airfoils specifically suited for unsteady flow, and which considers the significance of:

- dynamic stall repeatability and insensivity

o airfoil roughness

o delayed stall and soft stall

o performance and prediction

o rotor stability, and

o airfoil control devices.

Wind Inflow Models: The Development of Aerometeorology

Establish a new study termed aerometeorology, which will attempt to develop realistic inflow models for the assessment of unsteady effects, including the specific cases of: 
- unsteady, uniform inflow-or representation of the uniform gust front;

o unsteady nonuniform inflow-or representation of frozen turbulence;

- steady, nonuniform inflow-or representation of steady inflow fronts; and

- stochastic inflow-or representation of inflow in the frequency/wave number domain.

\section{Interface Topics}

Aeroelasticity--

Develop a methodology for defining the unsteady airloads which must be included in the structural dynamic models.

\section{Control Systems--}

Investigate the performance and reliability benefits to be obtained with external control actions, such as ailerons, in steady and unsteady flow.

Wake Model Interference Development--

Develop and verify a model for the structure and decay of the unsteady wind turbine rotor wake.

Component Wake Interference Development--

Develop and verify models for the structure and decay of the unsteady wakes caused by turbine components such as towers.
Shutdown (Emergency) Systems--

Develop an understanding of the behavior of airfoils under extreme conditions, and at very high angles of attack, such as a moving aileron in separated flow.

In addition, further steadystate aerodynamics studies should be continued in order to provide a suitable data base for the above investigations and lend insight into the new physical and mathematical models and design tools which must be used.

Steady Aerodynamics

Three-Dimensional Flow--

Assess the degree of 3-D or spanwise flow which occurs under typical conditions, and relate that to the inflow and turbine wake geometry.

Wake Modeling--

Develop and verify a model of the turbine vortex wake geometry suitable for performance and stability studies.

Effect of Roughness in Steady State--

Develop an understanding of environmentally-induced airfoil surface roughness on airfoil transition and separation in steady flow.

Airfoil Mechanisms--

Investigate the effect of airfoil mechanisms, such as vortex generators, on delaying turbine rotor stall and separation. 
Two-Dimensional Airfoil

Development--

Develop a verified methodology for tailored wind turbine airfoil design in steady state to establish a database for future unsteady airfoil design.

Testing Methodology for Steady Flow--

Continue steady-state wind turbine testing in wind tunnels and in the field, to acquire long-. term performance data and to investigate the benefits of new airfoils.

The Technical Assessment has thus provided the proper focus for specific tasks to meet the DOE objectives of improving the understanding of fundamental sources of wind variability, and increasing the basic understanding of the interactions between the wind, the air flow response, and the performance of wind energy systems. Further, the recommended work will eventually result in adequate and confident design tools for configuration studies, thus permitting major improvements in productivity and reliability of future wind energy systems.

\section{DIFFICULTY IN ESTABLISHING SPECIFIC TASK GOALS}

The specific goals of the individual investigations cannot and should not be described in detail here. Additional work must be done. The most that can be said at this time is that an effective plan will incorporate the following aspects, which are described more fully in Chapter 5:
- Stressing basic research,

o Striving for better understanding of the physical phenomena,

o Maintaining a patient, long-term attitude,

- Keeping distance from the commercial uses, and

o Coordinating knowledge from disciplines related to aerodynamics and affecting wind turbine design.

\section{MANAGEMENT OF INVESTIGATIONS}

The bulk of the R\&D investigations should ideally occur at a facility which would include both analytical and experimental (testing) studies. Subproblems properly defined by the overall $R \& D$ plan could be subcontracted to capable outside contractors, but their supervision, as well as the inhouse R\&D management, should be kept centralized. If the management planning is done by incorporating the aspects described above, there should be a minimum of program competition and conflict of interest since the scientific center of expertise for each task will be obvious in each case.

The reporting would be vertical in all cases; that is, a clear central administrative and decision-making of fice would control all aspects of the wind turbine program.

The experimental facility for field- and wind-tunnel testing should also ideally be located near the analysis facility to permit frequent and informal communication among researchers. A wide range of engineering and scientific disciplines characteristic of basic research would also be required.

Inhouse funding and technical staffing should be large enough to 
allow a wide degree of investigative freedom, but care should be taken to discourage proprietary, closed, or near-term commercializable projects. In summary the characteristics of the recommended $R \& D$ management would be:

1. Vertical reporting and accountability.

2. Close proximity of theoretical and experimental efforts.

3. Close cooperation of test and analysis researchers.

4. Central accountability of outside subcontractors.

5. Scheduled central review of R\&D goals and progress.

6. Scheduled frequent reporting of results to the technical community and industry.

7. Particular effort to keep a wide range of technical disciplines inhouse.

8. Maintenance of distance from the commercial interests, but encouragement of the cooperation of industry $R \& D$ efforts.

\section{FACILITY}

The best facility would ideally be a first-rate wind tunnel and a field test installation in close proximity. The wind tunnel would have unsteady flow and turbulence-generating capability. The field installation would have a sophisticated, permanent spatial array of sensors to record the atmospheric inflow statistics. Instrumentation for the wind tunnel and field site would be determined so as to accurately and completely record the variables needed to have good experimental control and an accurate frame of reference.

A sophisticated data-processing center would be required for both installations to permit accurate storage of data; real-time generation of turbulence statistics necessary for stochastic analysis; verification of state-of-the-art analytical codes; building up of a field test database for future retrieval; and manipulation of large data sets.

The field installation would have rotors with full aerodynamic and dynamic instrumentation which includes: blade spanwise and chordwise pressure transducers; blade strain transducers; rotor thrust and torque transducers; rotorblade motion and position indicators; and flow visualization capability. Collective and cyclic pitch positioning and recording would be required also, with the necessary frequency response determined by the appropriate unsteady aerodynamics test plan.

Aware that the above will be very difficult to achieve, the Panel recommends a next-best approach of subcontracting for the wind tunnel, and keeping the rest of the effort together at a common facility. 


\section{LIST OF REFERENCES}

1. ....., "Five Year Research Plan: 1985-1990", Wind Energy Technology Division, Office of Solar Electric Technologies, U.S. Department of Energy, DOE/CE-T11, January 1985 .

2. ....., "Wind Energy Systems: Program Summary for FY 1983", Solar Energy Research Institute, prepared for Asst. Secy., Conservation and Renewable Energy, U.S. Dept. of Energy, SERI/SP-271-2647, December 1984.

3. Klimas, P., et al, Wind Turbine Aerodynamics Seminar, informal proceedings, Sandia National Laboratories, Albuquerque, N.M., March 26-29, 1985.

4. Saunders, G.H., Dynamics of Helicopter Flight, John Wiley \& Sons, Inc., New York, 1975.

5. McGhee, R.J., Beasley, W.D., and Somers, D.M., "Low-Speed Aerodynamic Characteristics of a 13-Percent-Thick Airfoil Section Designed for General Aviation Applications", NASA Langley Research Center, Hampton, VA., NASA TM X-72697, May 1977.

6. Tangler, J.L., and Somers, D.M., "Advanced Airfoils for Small HAWT's", Presented at Wind Turbine Aerodynamics Seminar, Sandia Natl. Labs., Albuquerque, N.M., March 1985.

7. Liu, H.T., "Flow Visualization Study of the MOD-2 Wind Turbine Wake", Flow Industries, Inc., Battelle Pacific NW Lab., PNL-4535, June 1983.

8. Dommasch, D.O., Sherby, S.S., and Connolly, T.F., Airplane Aerodynamics, 4th Edition, Pitman Publishing Corp., New York, 1967.

9. Carr, L.W., McAlister, K.W., and McCroskey, W.J., "Analysis of the Development of Dynamic Stall Based on Oscillating Airfoil Experiments", NASA Ames Research Center, Moffett Field, CA, NASA TN D-8382, January 1977.

10. Carr, L.W., Unpublished test results, Air Force Office of Scientific Research, August, 1985.

11. Miley, S.J. , "A Catalog of Low Reynold's Number Airfoil Data for Wind Turbine Applications", Texas A\&M University, US DOE Report RFP-3387, February 1982.

12. Cermak, J.E., "Physical Modelling of Flow and Dispersion Over Complex Terrain", Boundary-Laver Meteorology 30(1984) pp. 261-292.

13. Wilson, R.E., and Walker, S.N., "Performance Analysis of Horizontal Axis Wind Turbines", Oregon State University, Corvallis, OR, NASA Lewis Research Center, Grant NAG-3-278, September 1984.

14. de Vries, O., "Comment of the Yaw Stability of a Horizontal-Axis Wind Turbine at Small Angles of Yaw", Wind Engineering, 9:1, National Aerospace Laboratory (NLR), The Netherlands, 1985. 
15. de Vries, O., "Fluid Dynamic Aspects of Wind Energy Conversion", AGARDograph No. 243, National Aerospace Laboratory (NLR), The Netherlands, 1979.

16. Lundsager, P., "On the Power Regulation of Small Wind Turbines Based on Experience with Small Danish Wind Turbines", Riso National Laboratory, Denmark, pres. at 5th Biennial Wind Energy Conference, Washington, D.C., October 1981.

17. Montgomerie, B., "Unsteady Aerodynamics Applied to the Horizontal Axis Wind Turbine Disk", National Aeronautical Laboratory, Sweden, FFA Memo, November 29, 1984.

18. Hirschbein, M.S., "Dynamics and Control of Large Horizontal-Axis Axisymmetric Wind Turbines", Ph.D. Thesis, Univ. of Delaware, University Microfilms 8006484, June, 1979.

19. Ericsson, L.E., "Is Any Free Flight/Wind Tunnel Equivalence Concept Valid for Unsteady Viscous Flow?", Lockheed Co. Inc., Sunnyvale, CA, Journal of Aircraft, Vol. 22, No. 10, October 1985, also AIAA Paper 85-0378.

20. Drees, H.M., FloWind Corp., Private testimony to the Wind Turbine Assessment Panel, Oakland, CA, August 1985.

21. Nelson, V. , Alternative Energy Institute, West Texas State Univ., Private testimony to the Wind Turbine Assessment Panel, Oakland, CA, August 1985.

22. Payne, F.M., and Nelson, R.C., "Aerodynamic Characteristics of An Airfoil in a Nonuniform Wind Profile", Univ. of Notre Dame, Notre Dame, IN, Journal of Aircraft, Vol. 22, No. 1, January 1985, also AIAA Paper 82-0214.

23. Mueller, T.T., "The Influence of Laminar Separation and Transition on Low Reynolds Number Airfoil Hysteresis", Univ. of Notre Dame, Notre Dame, IN, Journal of Aircraft, Vol. 22, No. 9, September 1985, also AIAA Paper 84-1617.

24. Staples, D.L., and Cao, H.V., "Summary of Wind Tunnel Results for the NACA 23024 Airfoil", Wichita St. Univ. Wichita, KS, prepared for NASA-Lewis Res. Center, Grant No. NSG-3277, WER-36, September 1985.

25. Akins, R.E., "Pressure Distributions on an Operating VAWT", Presented at Wind Turbine Aerodynamics Seminar, Sandia Natl. Labs., March 1.985.

26. .....Private comments shared, Windfarm Site Visits, Wind Turbine Assessment Panel Meeting, Oakland, CA, August 1985.

27. Bundas, D. and Dugundji, J., "Some Experiments on Yaw Stability of Wind Turbines with Various Coning Angles", MIT ASRL Report TR 197-2, July 1981.

28. Connell, J.R., and George, R.L., "Scaling Wind Characteristics for Designing Small and Large Wind Turbines", Battelle Pacific NW Lab., Presented at 6th Biennial Wind Energy Conference and Workshop, Minneapolis, MN, June 1-3, 1983. 
29. Hausfeld, T.E., and Hansen, A.C., "A Systematic Approach to Using the Method-of-Bins", U.S. Dept. of Energy, Small Wind Turbine Test Center, Rocky Flats, $\mathrm{CO}, 1983$.

30. Lundsager, P., Petersen, H., and Frandsen, S., "The Dynamic Behavior of the Stall-Regulated Nibe A Wind Turbine: Measurements and a Model for Stall-Induced Vibrations", Riso Report M-2253, Riso National Laboratory, Denmark, November 1981.

31. Eggleston, D.M., and Stoddard, F.S., Wind Turbine Engineering Design, Van Nostrand Reinhold, New York, to be published.

32. Althaus, D., "Polarenmussungen An Den Profilen Wortmann FX 84-W", Univ. of Stuttgardt, FRG, Presented at 12th Meeting of Aerodynamic Experts for Calculation Methods for WECS, Technical Univ. of Denmark, International Energy Agency IEA, ISSN 0343-7639, March 1985.

33. Wentz, W., and Snyder, M., "Wind Tunnel Tests of Spoilers and Ailerons for Wind Turbine Power Control and Braking", Presented at Wind Turbine Aerodynamics Seminar, Sandia Natl. Labs., Albuquerque, NM, March 1985.

34. Vermeulen, P., "A Wind Tunnel Study of the Wake of a Horizontal-Axis Wind Turbine", Netherlands Organization for Applied Sciences, Research TNO Report No. 78-09674, September 1978.

35. Lissaman, P.B.S., Gyatt, G.W., and Zalay, A.D., "Numeric Modeling Sensitivity Analysis of the Performance of Wind Turbine Arrays", AeroVironment, Inc., Pasadena, CA, prepared for Battelle Pacific NW Lab., PNL-4183, June 1982.

36. Vermeulen, P., "An Experimental Analysis of Wind Turbine Wakes", Presented at 3rd International Symposium on Wind Energy Systems, Copenhagen, Denmark, 1980.

37. Murtha, S.J., "Loads and Fatigue Evaluation of the Hamilton Standard WTS-4 Wind Turbine", Presented at WindPower '85, Conference of the American Wind Energy Association and U.S. Dept. of Energy, San Francisco, August 27-30, 1985.

38. Ardebili, M., Hazaraka, B.K., and Raj, R., "The Separated and Non-separated Airfoil Wake Behavior in the Presence of Free Stream Turbulence", C.C.N.Y., New York, Pres. at 3rd Applied Aerodynamics Conference, Colorado Springs, CO, October 14-16, 1985, AIAA Paper 85-5027.

39. Wentz, W., "Effects of Spanwise Flow on the Section Properties of a Stalled Airfoil", Presented at Wind Turbine Aerodynamics Seminar, Sandia Natl. Labs., Albuquerque, NM, March 1985.

40. Klimas, P.C., "A Spanwise Flow Estimation Model", Presented at Wind Turbine Aerodynamics Seminar, Sandia Natl. Labs., Albuquerque, NM, March 1985.

41. Marchman, J.F., Abtant, A.A., Sumantrau, V., and Sun, Z., "Effects of Aspect Ratio on Stall Hysteresis for the Wortmann Airfoil", V.P.I., Blacksburg, VA, AIAA Paper 851770, October 1985. 
42. Im, B.J., "Advanced Method for Modeling Airfoil Surface and Wake Panels in Unsteady Flow", Texas Tech University, Presented at Wind Turbine Aerodynamics Seminar, Sandia Natl. Labs., Albuquerque, NM, March 1985.

43. Miller, R.H., et al, "Free Wake Analysis of Wind Turbine Aerodynamics", Volume VIII, MIT ASRL TR-184-14, "Wind Energy Conversion", Prepared for U.S. Dept. of Energy, Sept. 1978.

44. Kocurek, J.D., "Lifting Surface Performance Analysis for Wind Energy Conversion System Rotors", Presented at Wind Turbine Aerodynamics Seminar, Sandia Natl. Labs., Albuquerque, NM, March 1985.

45. Miller, G.E., "The Effect of Vortex Generators on the Section Properties of a Modified NACA 23024 Airfoil", Boeing Aerospace Company, Presented at Wind Turbine Aerodynamics Seminar, Sandia Natl. Labs., Albuquerque, NM, March 1985.

46. Berg, D.E., "An Improved Double-Multiple Streamtube Model for the Darrieus-Type Vertical Axis Wind Turbine", Presented at 6th Biennial Wind Energy Conference and Workshop, U.S. Dept. of Energy, Minneapolis, MN, June 1-3, 1983.

47. Cheney, M.C., Windtech, Inc., Private comments at the Wind Turbine Assessment Panel Meeting, Oakland, CA, August 1985.

48. Powell, D.C., and Connell, J.R., "Definition of Gust Model Concepts and Review of Gust Models", Battelle Pacific NW Laboratory, PNL-3138, June 1980.

49. Panofsky, H.A., and Dutton, J.A., Atmospheric Turbulence, John Wiley \& Sons, New York, 1984.

50. Hoerner, S.F., Fluid-Dynamic Drag, Published by the author, 1958.

51. Rawson, K.J., and Tupper, E.C., Basic Ship Theory, American Elsevier Publishing Co., Inc., New York, 1968.

52. Lindenbaum, B.L., et a1, "Beyond The Horizon: Flight in the Atmosphere, 1975-1985", Volume III, "Vertical Takeoff and Landing", USAF Air Force Systems Command, AFSC Task Force, January 1967.

53. Martinez, R., Widnall, S.E., and Harris, W.L., "Predictions of Low Frequency and Impulsive Sound Radiation from Horizontal Axis Wind Turbines", NASA CR 2185, February 1981.

54. Ljungstrom, O., "Large Scale Wind Energy Conversion System (WECS) Design and Installation as Affected by Site Wind Energy Characteristics, Grouping Arrangement, and Social Acceptance", Wind Engineering 1:1, 1977.

55. Le Gourieres, D., Wind Power Plants: Theory and Design, Pergamon Press, Oxford, UK, 1982.

56. Rasmussen, F., "Blade and Rotor Loads for Vestas 15", Riso-M-2402, Test Plant for Small Windmills, Riso National Laboratory, Denmark, June 1983. 
57. Ormiston, R.A., "Rotor Dynamic Considerations for Large Wind Power Generator Systems", Presented at 1st Workshop Proceedings, Wind Energy Conversion Systems, National Science Foundation/RANN, Washington, D.C., June 11-13, 1973.

58. Hurt, H.H., Aerodynamics for Naval Aviators, Navair 00-80T-80, Revised January 1965.

59. van Holten, T., "Performance Analysis of a Windmill with Increased Power Output due to Tipvane Induced Diffusion of the Airstream", Memorandum M-224, Technische Hogeschool Delft, The Netherlands, November 1974.

60. Lissaman, P.B.S., Zalay, A.D., and Hibbs, B.H., "Advanced and Innovative Wind Energy Concept Development: Dynamic Inducer Systems", Executive Summary, AeroVironment, Pasadena, CA, May 1981.

61. Steeley, W., Hillesland, T., Ilyin, M, and Smith, D., "PGE's Wind Energy Program Results", Pacific Gas and Electric Company, Presented at Windpower '85, Conference of American Wind Energy Association and U.S. Dept. of Energy, San Francisco, CA, August27-30, 1985.

62. ....., "Federal Wind Energy Program, 5th Annual Revised Comprehensive Program Management Plan", draft November 1985, Office of Solar Electric Technologies, Conservation and Renewable Energy, US Dept. of Energy, Washington, DC.

63. Stoddard, F.S., "Momentum Theory and Flow States for Windmills", Wind Technology Journal, 1:1, Spring 1977.

64. Walker, S.N., and Wilson, R.E., "Performance Analysis Program for Propeller Type Wind Turbines", Oregon State University, Corvallis, OR, October 1976.

65. Wilson, R.E., and Lissaman, P.B.S., "Applied Aerodynamics of Wind Power Machines", Oregon State University, Corvallis, OR, NTIS PB 238594, July 1974.

66. Dugundji, J., and Wendell, J.H., "General Review of the MOSTAS Computer Code for Wind Turbines", MIT ASRL Report 197-1, NASA Lewis Research Center, NASA CR-165385, June 1981.

67. McCormick, B.W., Aerodynamics of V/STOL Flight, Academic Press, New York, 1967.

68. Mehta, U.B., "Dynamic Stall of an Oscillating Airfoil", Proceedings AGARD Fluid Dynamics Panel Symposium on Unsteady Aerodynamics, AGARD CPP-227, September 1977.

69. Nielsen, P., et al, "Measurements on the Nibe Wind Turbines: January 1980 to March 1981", Research Assn. of the Danish Electricity Supply Undertakings, DEFU Report No. EEV 81-04, May 1981. 
70. Cromack, D.E., et al, "Investigation of the Feasibility of Using Windpower for Space Heating in Colder Climates", Final Report, Energy Alternatives Program, Univ. of Mass., Amherst, MA, UM-WF-PR-78-5, July 1978.

71. Lynette, R., et al, "Wind Power Stations: 1984 Performance and Reliability", Electric Power Research Institute, Palo Alto, CA, Res. Proj. 1996-2, Interim Report, January 1985.

72. Glasglow, J.C., Corrigan, R.D. and Miller, D.R., "The Effect of Yaw on HAWT Loading and Performance" NASA-Lewis Research Center, DOE/NASA/20320-35, NASA TM-82778, presented at 5th Biennial Wind Workshop, Washington, D.C. October 5-7, 1980. 


\section{APPENDIX 1. LIST OF REPORTS REVIEWED}

1985:

Hibbs, B.D., "HAWT Performance with Dynamic Stall", AeroVironment, Inc., Pasadena, Calif., SERI, March 1985.

Swift, A.H.P., Editor, "Proceedings of Fourth ASME Wind Energy Symposium", Eighth Annual Energy Sources Technology Conference and Exhibition, Dallas, Texas, Feb. 17-21, 1985, American Society of Mechanical Engineers, 345 East 47th St., New York 10017.

Thresher, R.L., and Hershberg, E.L., "Development of an Analytical Model and Code for the Flapping Response of a HAWT Rotor Blade", Oregon State University, Corvallis, Oregon, SERI, February 1985.

Kelley, N.D., et al, "Acoustic Noise Associated with the MOD-1 Wind Turbine: Its Source, Impact, and Control", SERI/TR-635-1166, February 1985.

1984:

Anon........, "Wind Energy for a Developing World", Wind Energy Program Office, U.S. DOE, International Trade Administration, U.S. Dept. of Commerce, and American Wind Energy Association, DOE/CE-T12, 1984.

Holley, W.E., Thresher, R.W., and Lin, S.R., "Atmospheric Turbulence Inputs for Horizontal Axis Wind Turbines", Oregon State University, Corvallis, Oregon, SERI, October 1984.

Wilson, R.E., and Walker, S.N., "Performance Analysis of Horizontal Axis Wind Turbines", Dept. of Mechanical Engineering, Oregon State Univ., Corvallis, Oregon, Sept. 1984; work performed for NASA/Lewis Research Center, Grant NAG-3-278.

Anon....., "MOD-5A Wind Turbine Generator Program Design Report, Volumes I, II, and III: Executive Summary, Conceptual \& Preliminary Design, and Final Design \& System Description", General Electric Company, DOE/NASA/0153-1,2, and 3, NASA CR-174734, 5, \& 6, 84AEPD004, August 1984.

Miller, D.R., and Puthoff, R.L., "Aileron Controls for Wind Turbine Applications", DOE/NASA/20320-60, NASA TM-86867, August 1984.

McCabe, T.F., "Analysis of Wind Energy Systems for Selected Electric Utilities", Solar Energy Research Institute, Golden, Colorado, SERI/TR-2379, July 1984.

Sullivan, T.L., "Effect of Vortex Generators on the Power Conversion Performance and Structural Dynamic Loads of the MOD-2 Wind Turbine", NASA Lewis Research Center, DOE/NASA/20320-59, NASA TM-83680, June 1984.

Flaim, T., and Hock, S., "Wind Energy Systems for Electric Utilities: A Synthesis of Value Studies", SERI, Golden, Colorado, SERI/STR-211-2318, May 1984. 
Miller, D.R., and Corrigan, R.D., "Shutdown Characteristics of the MOD-0 Wind Turbine with Aileron Controls", DOE/NASA/20320-61, NASA TM-86919, May 1984.

Gregorek, G.M., "Design \& Wind Tunnel Evaluation of a Symmetric Airfoil Series for Large Wind Turbine Applications", Ohio State University, DOE/NASA/0330-1, NASA CR-174764, May 1984.

Baldwin, D.H., and Kennard, J.G., "DOE Large Horizontal Axis Wind Turbine Development at NASA Lewis", Sverdrup Technology Inc., Pres. at ASCE Spring Meeting, Atlanta, May 1984.

Tangler, J.L., and Ostowari, C., "Horizontal Axis Wind Turbine Post Stall Characteristics Synthesization", SERI/Texas A \& M University, SERI, May 1984.

Lynette, R., "Wind Turbine Operational Experience", Solar and Wind Power 1984 Status and Outlook, Extended Abstracts, Electric Power Research Institute, Palo Alto, California, March 14-16, 1984.

Linscott, B.S., Perkins, P., and Dennett, J.T., "Large, Horizontal-Axis Wind Turbines", NASA Lewis Research Center, DOE/NASA/20320-58, NASA TM-83546, March 1984.

George, R.L., "Simulation of Wind Speeds as Seen by a Rotating Vertical Axis Wind Turbine Blade", Battelle Pacific NW Labs., PNL-4914, Feb. 1984.

1983:

Veers, P.S., "A General Method for Fatigue Analysis of Vertical Axis Wind Turbine Blades", SAND-82-2543, October 1983.

Baldwin, D.H., and Linscott, B.S., "The Federal Wind Program at NASA Lewis Research Center", NASA TM-83430, Sept. 1983.

Dodge, D.M., and Liske, C., "Small Wind Systems Field Evaluation, Final Report: Volume I--Executive Summary and Program Description", U.S. DOE, Rocky Flats Small Wind Systems Test Center, RFP-3562/1, July 1983.

Sherman, J.M., Dodge, D.M., and Bollmeier, W.S., "Small Wind Systems Field Evaluation, Final Report: Volume IV--Small Wind Systems Performance Data", U.S. DOE, Rocky Flats Small Wind Systems Test Center, RFP-3562/4, July 1983.

Symanski, D.P., "Operating Experiences and Performance Characteristics During the First Year of Operation of the Crotched Mtn., New Hampshire, Windfarm", IEEE Transactions, Power Appar. Systems PAS-102(6): 1637-1641, June 1983.

Liu, H.T., Waite, J.W., Hiester, T.R., et al, "Flow Visualization Study of the MOD-2 Wind Turbine Wake", Battelle Pacific Northwest Lab., NTIS, PNL-4535, June 1983.

Goldenberg, J., and Fekete, G.I., "Mean Flow Energy Content of Boundary Layer Downstream of Vertical Axis Wind Turbine Simulators", Journal of Wind Eng. Ind. Aerodyn. 12(1): 1-14, June 1983. 
Miller, D.R., and Ensworth, C.B.F., "Analytical Model for Predicting Emergency Shutdown of a Two-Bladed Horizontal Axis Wind Turbine", Pres. at Wind Workshop VI, American Solar Energy Society, Minneapolis, June 1-3, 1983, DOE/NASA/20320-50, NASA TM-83472.

Beans, F.W., "Approximate Aerodynamic Analysis for Horizontal Axis Wind Turbines", $\underline{\text { J. }}$ Energy 7(3): 243-249, May-June 1983.

Shepherd, K.P., and Hubbard, H.H., "Measurements and Observations of Noise from a 4.2 MW (WTS-4) Wind Turbine Generator", Bionetics Corp., NASA Contractor Report 166124, May 1983.

Connell, J.R., and George, R.L., "Scaling Wind Characteristics for Designing Small and Large Wind Turbines", Battelle Pacific Northwest Lab., PNL-SA-10860, CONF-830622-21, NTIS, May 1983.

Hadley, D.L., and Renne, D.S., "Status of Wind Turbine Wakes Research in the Federal Wind Energy Program", Battelle Pacific NW Lab., PNL-SA-10999, CONF-830622-16, May 1983.

Kirchoff, R.H., and Kaminsky, F.C., "Empirical Modelling of Wind-Speed Profiles in Complex Terrain", Univ. of Mass., Amherst, Mass., D0E/ET/10374-82/1, April 1983.

Anon........, "Fiscal Year 1982 Annual Report: Rocky Flats Wind Energy Research Center", U.S. DOE, Rocky Flats Small Wind Systems Test Center, RFP-3612, April 1983.

Tu, P.K.C., and Kertesz, V., "SEACC: The Systems Engineering \& Analysis Computer Code for Small Wind Systems", Rocky Flats Small Wind Systems Test Center, Rockwell International, RFP-3510, March 1983.

Sullivan, T.L., "Structural Qualification Testing and Operational Loading on a Fiberglass Rotor Blade for the MOD-0A Wind Turbine", NASA Lewis Research Center, DOE/NASA/20320-44, NASA TM-83309, March 1983.

Lissaman, P.B.S., Zambrano, T.G., and Gyatt, G.W., "Wake Structure Measurements at the MOD-2 Cluster Test Facility at Goodnoe Hills", AeroVironment, Inc., Pasadena, Calif., PNL-4572, March 1983.

Tangler, J.L., et al, "Horizontal Axis Wind System Rotor Performance Model Comparison: A Compendium", U.S. DOE, Rocky Flats Wind Systems Test Center, Rockwell International, RFP-3508, February 1983.

Velkoff, H.R., "Fluid Dynamics Panel Specialists' Meeting on Prediction of Aerodynamic Loads on Rotorcraft", AGARD-AR-189, NTIS, Feb. 1983.

Noll, R.B., and Ham, N.D., "Dynamic Stall of Small Wind Systems", Aerospace Sciences, Inc., Burlington, Mass., RFP-3523, Feb. 1983.

Bossanyi, E.A., "Windmill Wake Turbulence Decay: A Preliminary Theoretical Model", SERI/TR-635-1280, NTIS, Feb. 1983. 
Mei-Kao, L., Yocke, M.A., and Myers, T.C., "Mathematical Model for the Analysis of Wind Turbine Wakes", Journal of Energy 7(1): 73-78, Jan.-Feb. 1983.

Vaschon, W.A., "Wind-Turbine Performance Assessment: Technology Status Report No. 5", A.D. Little, Inc., Prepared for Electric Power Research Institute, Palo Alto, California, EPRI-AP-2796, Jan. 1983.

Corrigan, R.D., and Ensworth, C.B.F., "Performance Comparison Between NACA 23024 and NACA 64(3)-618 Airfoil Configured Rotors for Horizontal Axis Wind Turbines", NASA Lewis Research Center, NASA TM-83471, 1983.

Janetzke, D.C., and Kaza, K.R.V., "Whirl Flutter Analysis of a Horizontal-Axis Wind Turbine with a Two-Bladed Teetering Rotor", NASA Lewis Research Center, Solar Energy 31(2): 173-182, 1983.

Berg, D.E., "Improved Double-Multiple Streamtube Model for the Darrieus-Type Vertical-Axis Wind Turbine", SAND-82-2479C, CONF-830622-8, 1983.

Jones, C.N., "Blade Element Performance in Horizontal Axis Wind Turbine Rotors", Wind Eng. 7(3): 129-137, 1983.

1982:

Hubbard, H.H., and Shepherd, K.D., "Noise Measurements for Single and Multiple Operation of $50 \mathrm{KW}$ Wind Turbine Generators", College of William and Mary, Bionics Corp., NASA CR-166052, December 1982.

Jeng, D.R., Keith, T.G., Jr., and Aliakbarkhanafjeh, A., "Aerodynamic Analysis of a Horizontal Axis Wind Turbine by Use of Helical Vortex Theory, Volume I: Theory", University of Toledo, Toledo, Ohio, NASA CR-168054, Dec. 1982.

Thresher, R.W., Holley, W.E., Hershberg, E.L., and Lin, S.R., "Response of the MOD-0A Wind Turbine Rotor to Turbulent Atmospheric Wind", NTIS, DOE/RL/10378-82/1, Dec. 1982.

Connell, J.R., and George, R.L., "Wake of the MOD-0A1 Wind Turbine at Two Rotor Diameters Downwind on 3 December 1981", Battelle Pacific NW Labs., PNL-4210, Nov. 1982.

Doran, J.C., and Packard, K.R., "Comparison of Model and Observations of the Wake of a MOD-0A Wind Turbine", Battelle Pacific NW Labs., PNL-4433, Oct. 1982.

Schefter, J.L., "Capturing Energy From the Wind", NASA Scientific and Technical Information Branch, Washington, D.C., 1982, NASA SP-455.

Dixon, J.C., "Wind Turbine Array Performance: The Influence of Wind Speed and Direction", Pres. at the 4th International Symposium on Wind Energy Systems, Stockholm, Sweden, Sept. 21, 1982, Bedford, England, BHRA Fluid Engineering, 1982, Volume 2, CONF-820922-2.

Builtjes, P.J.H., and Vermeulen, P.E.J., "Turbulence in Wind-Turbine Clusters", Ibid. 
Dugundji, J., "Summary Technical Report on NASA Grant No. NSG-3303, 'Development of a Methodology for Horizontal Axis Wind Turbine Dynamic Analysis"', MIT, Cambridge, Mass., ASRL TR-197-5, DOE/NASA/3303-4, NASA CR-168110, Sept. 1982.

Wendell, J.H., "Simplified Aeroelastic Modeling of Horizontal Axis Wind Turbines", MIT, Cambridge, Mass., ASRL TR-197-4, DOE/NASA/3303-3, NASA CR-168109, Sept. 1982.

Anon........, Boeing Engineering and Construction, "MOD-2 Wind Turbine System Development Final Report, Volume I: Executive Summary, and Volume II: Detailed Report", DOE/NASA/0002-82/1 and 2, NASA CR-Nos. 168006 and 168007, Sept. 1982.

Viterna, L.A., and Janetzke, D.C., "Theoretical and Experimental Power from Large Horizontal Axis Wind Turbines", DOE/NASA/20320-41, NASA TM-82944, Sept. 1982.

Anon...... "Prediction of Aerodynamic Loads on Rotorcraft", AGARD Fluid Dynamics Panel Specialists's Meeting, London, May 17-18, 1982, AGARD-CP-334, NTIS, Sept. 1982.

Kamoulakos, A., "Stability Analysis of Flexible Wind Turbine Blades Using Finite Element Method", MIT, Cambridge, Mass., ASRL TR-197-3, DOE/NASA/3303-2, NASA CR-168107, August 1982.

Kristensen, L., and Frandsen, S., "Model for Power Spectra of the Blade of a Wind Turbine Measured From the Moving Frame of Reference", J. Wind Eng. and. Aerodyn. 10(2): 249-262, August 1982.

Miller, R.H., "Simplified Free Wake Analysis for Rotors", MIT, Cambridge, Mass., ASRL-TR-194-3, NTIS, FFA-TN-1982-07, August 1982.

Sherman, J.M., et al, "Wind Systems Life Cycle Cost Analysis, A Description and Users Manual", Rocky Flats Small Wind Systems Test Center, Rockwell International, July 1982.

Vaschon, W.A., "Wind-Turbine Performance Assessment: Technology Status Report No. 4", A.D. Little, Inc., Prepared for Electric Power Research Institute, Palo Alto, California, EPRI-AP-2456, June 1982.

Raab, A., Winzell, B., and Hjorth, U., "Fatigue Evaluation of Wind Turbines: Part 1, Theoretical Background", NTIS, FFA-TN-1982-09, June 1982.

Migliore, P.G., and Fritschen, J.R., "Darrieus Wind-Turbine Airfoil Configurations", SERI/TR-11045-1, June 1982.

Lissaman, P.B.S., Gyatt, G.W., and Zalay, A.D., "Numeric Modelling Sensitivity Analysis of the Performance of Wind Turbine Arrays", AeroVironment, Inc., Pasadena, Calif., Report PNL-4183, SERI ENTR 1982-0027, June 1982.

Anon........, Technical Assessment Guide, Electric Power Research Institute, Palo Alto, Calif., EPRI-P-2410-SR, May 1982.

Van Bussel, G.J.W., Van Holten, T., and Van Kuik, G.A.M., "Aerodynamic Research on Tip Vane Wind Turbines", VTH-LR-355, NTIS, N83-11603, April 1982. 
Schuler, S.A., "Wind Systems Technical Notes: 1981", U.S. DOE, Rocky Flats Wind Systems Test Center, RFP-3383, March 1982.

Bielawa, R.L., "Development of an Oscillating Vane Concept as an Innovative Wind Energy Conversion System", UTRC, Hartford, Connecticut, SERI/TR-98085-2, NTIS, March 1982.

Sullivan, R.L., Flaim, T., and Percival, D., "WECS Value Analysis: A Comparative Assessment of Four Methods", SERI/TR-211-1563, March 1982.

Percival, D., and Harper, J., "Electric Utility Value Determination for Wind Energy", Volumes 1 \& 2, SERI/TR-732-604R, January 1982.

Corrigan, R.D., Glasgow, J.C., and Sirocky, P.J., "Measured Performance of a Tip-Controlled, Teetered Rotor with an NACA 64(3)-618 Tip Airfoil", NASA Lewis Research Center, Cleveland, Ohio, DOE/NASA/20320-40, NASA-TM-82870, CONF-820423-2, 1982.

Sullivan, T.L., "A Review of Resonance Response in Large, Horizontal-Axis Wind Turbines", NASA Lewis Research Center, Solar Energy 29(5): 377-383, 1982.

Snyder, M.H., "Further Investigations of Near-Field Wakes of Circular and 12-Sided Cylinders and Effects of Shrouds and Strakes", Wichita State University, Grant No. NSG-3277, DOE/NASA/3277-3, NASA CR-168169, 1982.

$1981:$

Batesole, W.R., and Gunsallus, C.T., "Design \& Fabrication of Composite Blades for the MOD-1 Wind Turbine Generator", Kaman Aerospace Corp., Bloomfield, Connecticut, DOE/NASA/0131-1, NASA CR-167987, Nov. 1981.

Hofer, K.E., and Bennett, L.C., "Fatigue Testing of Low-Cost Fiberglass Composite Wind Turbine Blade Materials", IIT Research Institute, DOE/NASA/0182-1, NASA CR-165566, Nov. 1981.

Connell, J.R., "Spectrum of Wind Speed Fluctuations Encountered by a Rotating Blade of a Wind Energy Conversion System: Observations and Theory", Battelle Pacific NW Lab., PNL-4083, NTIS, Nov. 1981.

Thomas, R.L., and Baldwin, D.H., "The NASA Lewis Large Wind Turbine Program", Pres. at Fifth Biennial Wind Energy Conference and Workshop, Washington, D.C., Oct. 5-7, 1981, DOE/NASA/20305-7, NASA TM-82761, NTIS N82-16495.

Tangler, J.L., "Comparison of Wind Turbine Performance Prediction and Performance", Ibid.

Weingart, O., "Design, Evaluation, and Fabrication of Low-Cost Composite Blades for Intermediate-Size Wind Turbines", Structural Composite Industries, Inc., Special Report 81520, DOE/NASA CR-165342, NTIS, N82-18693, Sept. 1981.

Sforza, P.M., Sheerin, P., and Smorto, M., "Three-Dimensional Wakes of Simulated Wind Turbines", AIAA Journal 19(9): 1101-107, Sept. 1981. 
Higashi, K.K., "UTRC 8-KW Prototype Wind System: Final Test Report", U.S. DOE Rocky Flats Small Wind Turbine Test Center, RFP-3294, Sept. 1981.

Thresher, R.W., Holley, W.E., et al, "Modeling the Response of Wind Turbines to Atmospheric Turbulence", DOE/ET/23144-81/2, NTIS, August 1981.

Jesch, L.F., Editor, "Proceedings of the International Colloquium on Wind Energy: 1981", BHRA Fluid Engineering, Brighton, England, Aug. 27-28, 1981, SERI ENTR 1981-0581.

Viterna, L.A., and Corrigan, R.D., "Fixed Pitch Rotor Performance of Large Horizontal Axis Wind Turbines", NASA Lewis Research Center, NASA/DOE Workshop, July 28-30, 1981, NASA Conference Pub. 2230.

Birchenough, A.G., et al, "Operating Experience with Four 200-KW MOD-OA Wind Turbine Generators", Ibid.

Spera, D.A., and Janetzke, D.C., "Performance and Load Data from MOD-0A and MOD-1 Wind Turbine Generators", Ibid.

Glasgow, J.C., and Corrigan, R.D., "Stall Induced Instability of a Teetered Rotor", Ibid.

Seidel, R.C., and Pfanner, H.G., "Tests of an Overrunning Clutch in a Wind Turbine", NASA Lewis Research Center, DOE/NASA/20320-32, NASA-TM-82653, July 1981.

Milhail, A.S., "Wind Power for Developing Nations", SERI/TR-762-966, July 1981.

Bundas, D., and Dugundji, J., "Some Experiments on Yaw Stability of Wind Turbines with Various Coning Angles: Final Report", MIT, Cambridge, Mass., Grant NSG-3303, ASRL TR-197-2, DOE/NASA/3303-1, NASA-CR-168108, July 1981.

Dugundji, J., and Wendell, J.H., "General Review of the MOSTAS Computer Code for Wind Turbines", MIT, Cambridge, Mass., ASRL TR-197-1, DOE/NASA/3303-1, NASA CR-165385, June 1981.

Lissaman, P.B.S., Zalay, A.D., and Hibbs, B.H., "Advanced and Innovative Wind Energy Concept Development: Dynamic Inducer System, Executive Summary", AeroVironment, Pasadena, Calif., SERI/TR-8085-1-T1, May 1981.

Thresher, R.W., and Holley, W.E., "The Response Sensitivity of Wind Turbines to Atmospheric Turbulence", DOE/ET/23144-81/1, NTIS, May 1981.

Senior, T.B.A., and Sengupta, D.L., "Large Wind Turbine Siting Handbook: Television Interference Assessment", Univ. of Michigan, Tech. Report No. 4, 014438-4-T, April 1981.

Noll, R.B., and Ham, N.D., "Study of Dynamic Stall as it Affects Small Wind Energy Systems Design", Aerospace Systems, Inc., Burlington, Mass., DOE Contract No. DE-AC04-76DP03533, April 1981.

Anon......., "Technical Description of the Nibe Wind Turbines", Test Station for Small Wind Turbines, Riso, Denmark, EEV-81-02, NTIS, April 1981. 
Cheney, M.C., "UTRC 8-KW Wind Turbine Tests", J. Energy 5(2): 12-128, March-April 1981.

Kareem, A., Lissaman, P.B.S., and Zambrano, T.G., "Wind Loading Definition for the Structural Design of Wind Turbine Generators", AeroVironment, Inc., Pasadena, California, J. Energy 5(2): 89-93, March-April 1981.

McCrosky, W.J., "The Phenomenon of Dynamic Stall", NASA TM-81264, March 1981.

Wentz, W.H. Jr., and Calhoun, J.T., "Analytical Studies of New Airfoils for Wind Turbines", Wichita State University, Pres. at Wind Turbine Dynamics Conference, Feb. 24-26, 1981, NASA Conference Publication 2185.

Egolf, T.A., and Landgrebe, A.J., "The UTRC Wind Energy Conversion System Performance Analysis for Horizontal Axis Wind Turbines (WECSPER)", United Technologies Research Center, East Hartford, Connecticut, Ibid.

Paraschivoiu, I., "Double-Multiple Streamtube Model for Darrieus Wind Turbines", Ibid.

Glasgow, J.C., Miller, D.R., and Corrigan, R.D., "Comparison of Upwind and Downwind Rotor Operations of the DOE/NASA 100-KW MOD-0 Wind Turbine", Ibid.

Van Holten, T., "Concentrator Systems for Wind Energy, With Emphasis on Tipvanes", Wind Engineering 5(1): 29-45, 1981.

1980:

Hirschbein, M.S., and Young, M.I., "Stability of Large Horizontal-Axis Axisymmetric Wind Turbines", NASA Lewis Research Center, Pres. at 3rd Miami International Conf. on Alternative Energy Sources, December 15-17, 1980, NASA TM-81623.

Powell, D.C., and Connell, J.R., "Definition of Gust Model Concepts and Review of Gust Models", Battelle Pacific NW Laboratory, PNL-3138, June 1980.

Wentz, W.H., Jr., Snyder, M.H., and Calhoun, J.T., "Feasibility Study of Aileron and Spoiler Control Systems for Large Horizontal Axis Wind Turbines", Wichita State University, Grant No. NSG 3277, DOE/NASA/3277-1, WER-10, NASA CR-159855, May 1980.

Gustafsson, A., Lundgren, S., and Frisk, B., "Application of a Method for Aerodynamic Analysis and Design of Horizontal Axis Wind Turbines", Sweden, NTIS, FFA-AU-1499, NE-VIND-80-11, May 1980.

Glasgow, J.C., and Miller, D.R., "Teetered, Tip-Controlled Rotor: Preliminary Test Results from MOD-0 100-Kw Experimental Wind Turbine", NASA Lewis Research Center, NASA TM-81445, April 1980.

Klimas, P.C., "Possible Aerodynamic Improvements for Future VAWT Systems", VAWT Design Technology Seminar for Industry, Albuquerque, N.M., Proceedings SAND-80-0984, April 1-3, 1980. 
Andersen, P.S., Lundsager, P., Peterson, H., and Krabbe, U., "Basic Theoretical Calculations of Wind Turbines", in Danish, Test Site for Small Windmills at Riso, Denmark, RISO-M-2153, NTIS, Jan. 1980.

Sexton, J.H., "North Wind Eagle 3 Wind Turbine Generator: Final Test Report", U.S. DOE Rocky Flats Small Wind Turbine Test Center, Rocky Flats Report RFP-3071, Jan. 1980.

Ly, K.H., and Chasteau, V.A.L., "Experiments on an Oscillating Airfoil and Applications to Wind Energy Converters", AIAA Paper 80-0524, 1980.

1979:

Gewehr, H.W., "Design, Fabrication, Test, and Evaluation of a Prototype 150-Foot Long Composite Wind Turbine Blade", Kaman Aerospace Corp., Bloomfield, Conn., DOE/NASA/0600-79/1, R-1575, NASA CR-159775, NTIS, N80-17548, Sept. 1979.

Lynette, R., and Poore, R., "MOD-2 Failure Mode and Effects Analysis", Boeing Engineering and Construction Co., Contract DEN3-2, DOE/NASA/0002-79/1, NASA CR-159632, July 1979.

Ramler, J.R., and Donovan, R.M., "Wind Turbines for Electric Utilities: Development Status and Economics", Pres. at Terrestrial Energy Systems Conference, AIAA, Orlando, Florida, June 4-6, 1979, DOE/NASA/1028-79/23, NASA TM-79170, NTIS, N79-30719.

Robbins, W.H., and Thomas, R.L., "Large Horizontal Axis Wind Turbine Development", Pres. at Wind Energy Innovative Systems Conference, SERI, Colorado Springs, Colorado, May 23-25, 1979, DOE/NASA/1059-79/2, NASA TM-79174, NTIS, N79-26504.

1978:

Gohard, J.C., "Free Wake Analysis of Wind Turbine Aerodynamics", MIT, Cambridge, Mass., ASRL-TR-184-14, NTIS, Sept. 1978.

Miller, R.H., Dugundji, J., Chopra, I., et al, "Dynamics of Horizontal Axis Wind Turbines: Wind Energy Conversion", MIT, Cambridge, Mass., ASRL-TR-184-9, COO-4131-T1(Vol. 3), Sept. 1978.

Sengupta, D.L., and Senior, T.B.A., "Electromagnetic Interference by Wind Turbine Generators: Final Report", University of Michigan, TID-28828, March 1978.

Senior, T.B.A., "Wind Turbine Generator Siting and TV Reception Handbook", University of Michigan, COO-2846-1, Jan. 1978.

Previous:

Hoffman, J.A., "Coupled Dynamics Analysis of Wind Energy Systems", Paragon Pacific, Inc., El Segundo, Calif., Contract NAS 3-19767, NASA CR-135152, February 1977.

Tryon, H., and Richards, T., "Installation and Initial Operation of a 4100 Watt Wind Turbine", NASA Lewis Research Center, ERDA/NASA 1004-77/10, NASA TM X-71831, December 1975. 
Eldridge, F.R., "Wind Machines", Prepared for National Science Foundation, RANN, Grant No. AER-75-12937, MITRE Corp., NSF-RA-N-75-051, Library of Congress, U.S. Government Printing Office: 1976, O-204-143, October 1975.

Wilson, R.E., et al, "Rigid Body Model for Analysis of Aerogenerator Rotor Dynamics", Preprint No. S991, Pres. at 31st Annual Forum, American Helicopter Society, Washington, D.C., May 1975.

McCloud, J.L., and Biggers, J.C., "How Big is a Windmill? Glauert Revisited", Ibid.

Rohrbach, C., and Worobel, R., "Performance Characteristics of Aerodynamically Optimum Turbines for Wind Energy Generators", Ibid.

Savino, J.M., Editor, "Wind Energy Conversion Systems", A Workshop Sponsored by NSF, RANN, and NASA, ERDA/NASA 4010-77/1, NASA TM X-69788, December 1973.

Wheatley, J.B., and Bioletti, C., "Wind Tunnel Tests of a 10-Foot Diameter Gyroplane Rotor", NACA TR-536, 1935.

Wheatley, J.B., and Hood, M.J., "Full-Scale Wind-Tunnel Tests of a PCA-2 Autogyro Rotor", NACA TR-515, 1934.

Lock, C.N.H., and Townend, H.C.H., "Wind Tunnel Experiments on a Model Autogyro at Small Angles of Incidence", British Aeronautical Research Council Reports and Memoranda, No. 1154, March 1928. 


\section{APPENDIX 2. A TUTORIAL: THE INFLUENCE OF AERODYNAMICS ON WIND TURBINE DESIGN}

This Appendix is a brief introduction on the influence of aerodynamics to wind turbine design, and can be referred to by readers who are unfamiliar with the wind turbine concepts and definitions used in the body of the Technical Needs Assessment.

\section{ROTOR CHARACTERISTICS}

The rotor is the prime mover of the system and everything else is slaved to it. The rotor is of ten the weight, control, and stiffness design driver for the rest of the design. Typically, save $1 \mathrm{lb}$. of rotating blade weight, and you'll save $10 \mathrm{lb}$. of tower and supporting structure. The function of the rotor is to produce rotational shaft energy from the fluid momentum of the wind; but this rotational energy, and its particular torque/speed characteristic, is adjustable by design over a wide range of possibilities in both the horizontal (HAWT) and vertical axis (VAWT) wind turbine approaches.

Since the flow is turbulent, the dynamics of the rotor dominate the activity of the system. The design environment is the classic aeroelastic triangle: inertial loads are due to mass and motion, aerodynamic loads are the driving and damping forces, and the elastic restraints are due to structural stiffnesses and control system programming.

The inertial loads are the best understood. Basic mechanics is well described by Newtonian principles, with a large body of knowledge and history. The elastic restraints are also well described by theory and proven experimentation. However, the aerodynamic loads are not well understood. Aircraft design methodology, especially for rotary-wing aircraft, depends still in large part on empirically-determined aerodynamic loads. The infinitesimal friction effects dominate, rather than the mass effects, and the nonlinearities of viscosity and compressibility are significant. These are inherently more complex, more difficult to measure, and more difficult to understand.

Aerodynamic forces are linear only within a small operating range, and then only in steady-state conditions. Aircraft generally operate within this linear range. Wind turbines must operate outside this linear range as well as within to be cost effective. The hysteresis effects of unsteady aerodynamics are not yet well described. Unsteady aerodynamic forces are dependent on their time histories as well as amplitudes. Pilots use the familiar delayed stall and increased dynamic lift to extend their performance in maneuvers. Wind turbine rotors see these unsteady events in a frequent, aggravating manner.

The wind turbine rotor is vulnerable to its environment. Compared to aircraft, the wind turbine mission envelope is very wide. The set of input conditions is more like an ocean-going sailing ship than a helicopter. Ocean engineering design methods historically have emphasized stochastic (statistical) descriptions rather than deterministic, in order to account for the extreme variety and randomness of conditions to be expected.

The turbulence of the wind flow is the most significant environmental driver for the wind turbine rotor. But other environmental conditions also drive the reliability: storms, high winds, precipitation, humidity, dust, temperature extremes, lightning, and ice combine with turbulence to present a formidable "input" to the rotor. 
The rotor can be thought of as having an "equilibrium" condition which is driven by the "input". For aircraft the "equilibrium" condition is at or near stable equilibrium. For a wind turbine, this condition may be far from stable equilibrium, at a condition which is closer to a superposition in mid-cycle of many large transient events.

Dynamic instabilities have been observed, and some have been duplicated in field testing with wind turbines, given the same "input" condition. The inertial and elastic parts of the formulation have even been done in some instability cases, but the unsteady aerodynamics is much more complex, and its development has lagged behind.

\section{ROTOR AERODYNAMIC DESIGN}

This Section examines the elementary aerodynamic theory and physics of the conventional rotor in steady state. The true equilibrium aerodynamic state of the rotor, though a fiction in field practice as pointed out above, is necessary to build an understanding of the more complex effects. Design practice, as in the aircraft industry, begins at this point to describe the equilibrium conditions and then adds the perturbations of the mission spectrum: turbulence, control inputs, and motion transients.

\section{Rotor Flow States}

The wind turbine rotor is usually described by the nondimensional parameters thrust coefficient, torque coefficient, and power coefficient, $\mathrm{CT}, \mathrm{CQ}, \mathrm{CP}$ :

$$
\begin{aligned}
& \text { Thrust Coefficient }=\frac{\text { Rotor Thrust }}{(\mathrm{q})(\mathrm{A})} \\
& \text { Torque Coefficient }=\frac{\text { Rotor Torque }}{(\mathrm{q})(\mathrm{A})(\mathrm{R})} \\
& \text { Power Coefficient }=\frac{\text { Rotor Power }}{(\mathrm{q})(\mathrm{A})\left(\mathrm{V}_{0}\right)}
\end{aligned}
$$

$$
\text { Where: } \quad \begin{aligned}
& \mathrm{A}=\text { Rotor Area } \\
& \mathrm{q}=\text { Dynamic Pressure }=1 / 2(\mathrm{Rho}) \mathrm{V}_{0}{ }^{2} \\
& \mathrm{~V}_{0}=\text { Free Stream Wind Speed } \\
& \mathbf{R}=\text { Rotor Radius }
\end{aligned}
$$

These are defined differently from the normal helicopter usage since the reference velocity for wind turbines is Vo, the free stream velocity, and for helicopters the reference velocity is tip speed. Therefore:

$$
\begin{aligned}
& \underset{\text { (Windmill) }}{\mathrm{CT}}=2 \times \underset{(\text { Helicopter })}{\mathrm{CT}} \times \text { (Tip Speed Ratio) }^{2} \\
& \underset{\text { (Windmill) }}{\mathrm{CQ}}=2 \times \underset{(\text { Helicopter })}{\mathrm{CQ}} \times \text { (Tip Speed Ratio) }^{2} \\
& \underset{(\text { Windmill) }}{\mathrm{CP}}=2 \times \underset{\text { (Helicopter) }}{\mathrm{CP}} \times \text { (Tip Speed Ratio) }^{3}
\end{aligned}
$$


Where:

Tip Speed Ratio = (Rotor speed in $\mathrm{Rad} / \mathrm{sec}) \times($ Radius $) / \mathrm{V}_{0}$

A rotor has a number of possible flow states, which determine whether it is a driving rotor (propeller) or a driven rotor (windmill). When the rotor in steady flow is completely uncoupled from its shaft load, it will speed up until the blade element angle has positive angle of attack with the incident velocity vector (see Figure A-1 [31]). The lift vector is providing just enough forward component to produce just enough torque to overcome friction in the system. This is the Zero Slip Case. It is clear from the diagram that increasing blade pitch angle will reduce the RPM necessary, and the lift necessary, to produce zero slip. The fully-feathered, slightly rotating, rotor would also be in zero slip with much smaller dynamic pressure, rotor lift, and thrust than shown in the diagram.

Referring to the blade element diagram, if the RPM is slightly reduced, angle of attack increases, lift on the blade increases, induced velocity increases, and torque also increases. This is the Normal Working State where the turbine produces power to its load. In the helicopter literature this is called the Windmill State.

If RPM speeds up, angle of attack reverses, the induced velocity and thrust are now in the upwind direction, and power is required to drive the rotor. This is the Propeller State where rotary wing aircraft operate (see Figure A-1).

Helicopter rotors operate in Propeller State when in vertical climb, and slightly in Windmill Brake State for vertical descent, where the power required can be very low, or zero or even negative in the case of autogiros. Wind turbines operate strictly in Windmill State at very high "rates of descent" and relatively low thrust, compared to helicopters and autogiros.

As seen in Figure A-2 [63], the Turbulent Wake State is another possibility, which occurs when the induced velocity and rotor thrust become very large. This occurs in autogiros and in autorotating helicopters which are attempting to increase thrust to slow descent. 


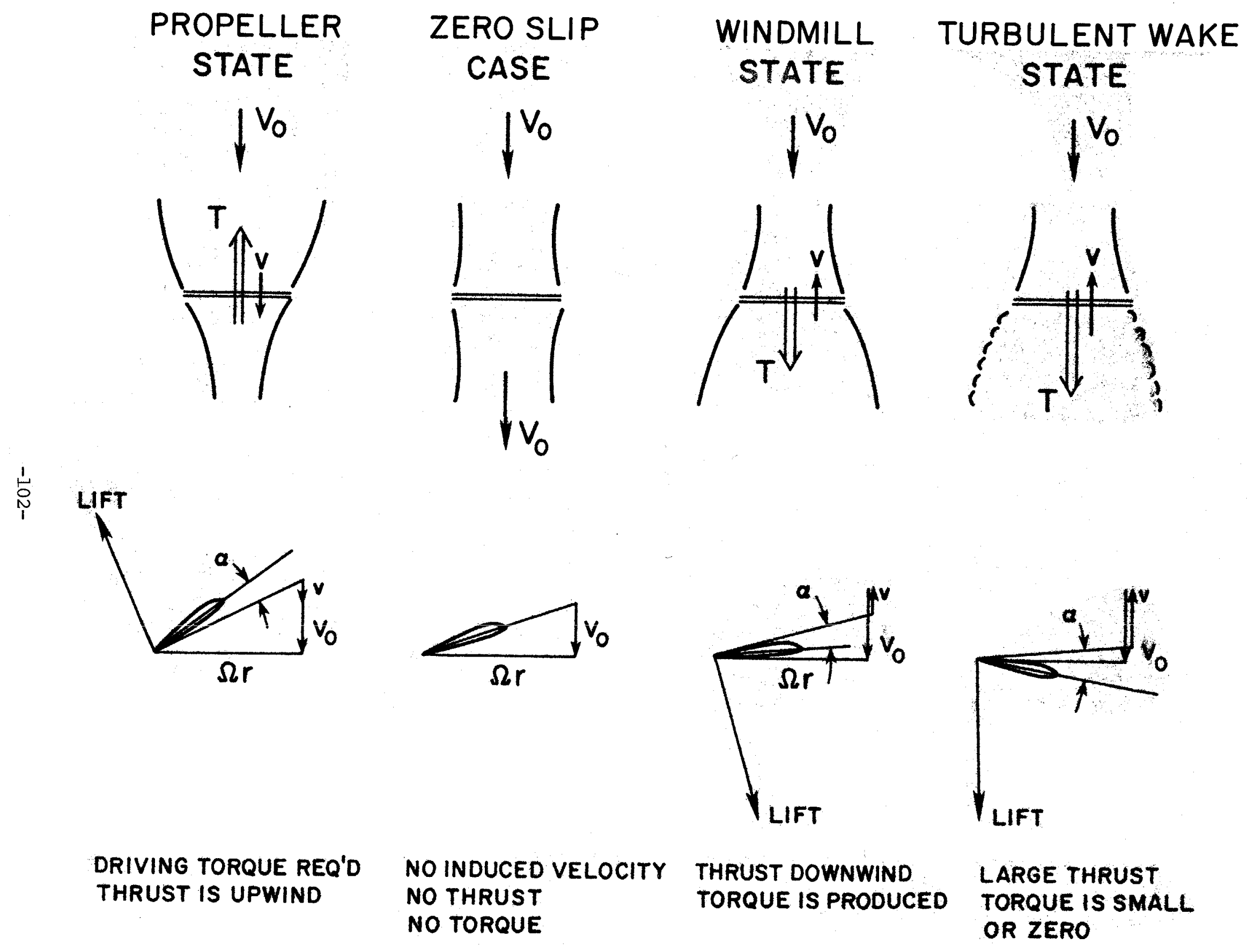


FIGURE A-2. ROTOR WAKE STATES [63]

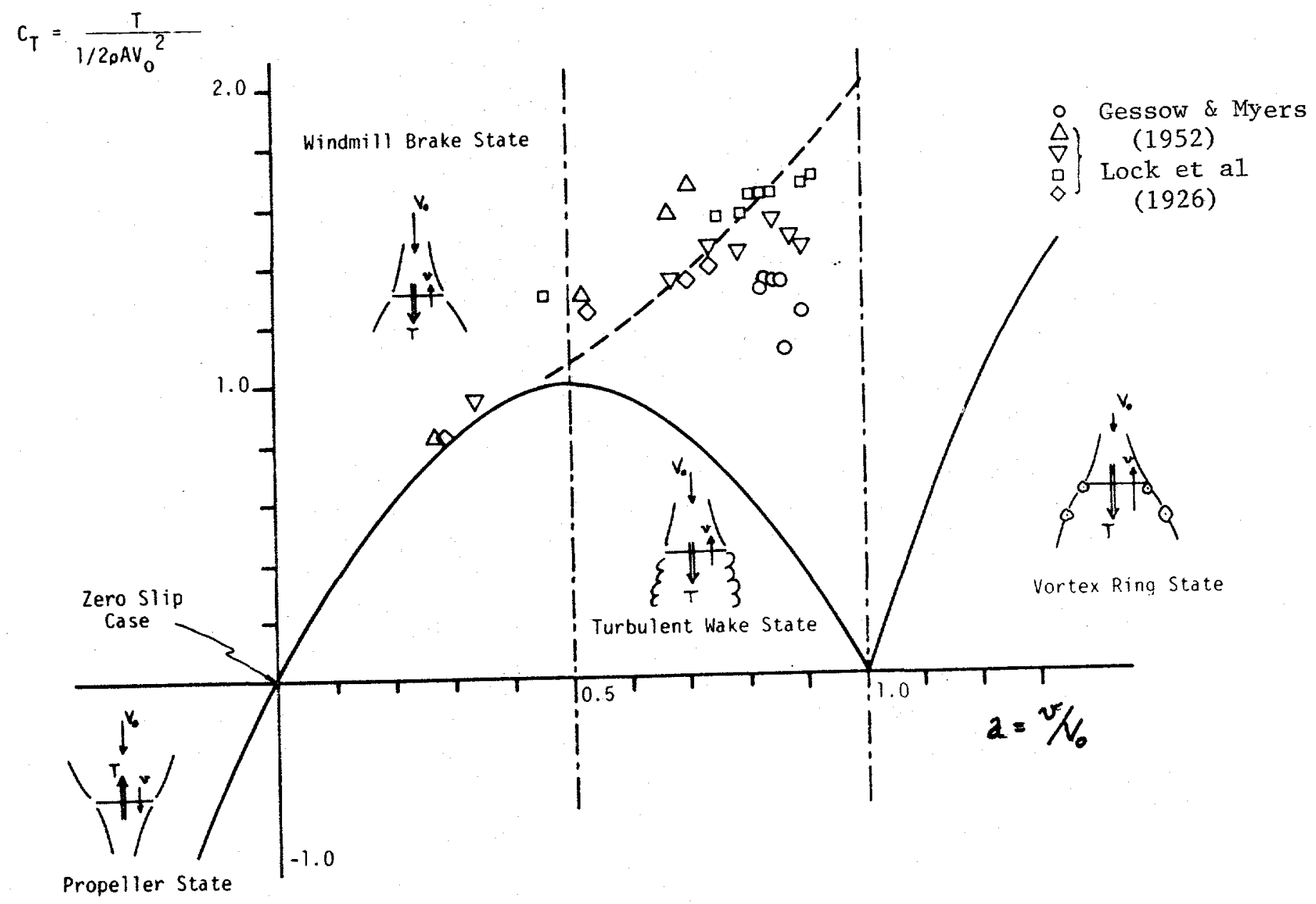




\section{Momentum Theory}

If the blade element description is replaced by a uniform "actuator disc" which produces uniform induced velocity over the rotor plane (see Figure A-2), the mathematical interpretation is called momentum theory. At zero slip, thrust $=0$, and $C T=0$ as before. Thrust is produced by the induced velocity, which is described nondimensionally as

$$
a=\underbrace{\text { induced }}_{\text {velocity }}=\frac{\text { induced velocity }}{\text { free stream, } V_{O}}
$$

The general results of momentum theory for the rotor are familiar to rotary wing designers, and momentum theory remains very useful in the analysis of steady flow:

1. Zero slip is at the origin; Propeller State is to the left, where thrust and power coefficients are negative, and Windmill State is to the right, where thrust and power coefficients are positive.

2. The torque and power maximum occurs at induced velocity $=1 / 3 \mathrm{X}$ free stream velocity, or $a=1 / 3$.

3. The thrust maximum occurs at induced velocity $=1 / 2 \mathrm{X}$ free stream, or $\mathrm{a}=$ $1 / 2$. The physical interpretation of this is that the induced velocity is then high enough to bring the free stream convection to a complete stop in the wake behind the rotor. This physical effect is of ten observed in wind turbines.

4. When induced velocity a is low, angle of attack, thrust and power are low; when $a$ is increased, these also increase.

5. When a reaches $1 / 2$, momentum theory no longer applies since the far wake velocity has vanished, hence streamlines have vanished.

\section{Blade Element Theory}

The most widely used predictors for wind turbine rotor performance and aerodynamic load specification are the blade element theories, which simply stated, are approximate methods usually in the form of computer codes which are used to predict the rotor performance using reference airfoil data $[64,65]$. Sophistication of these strip theories varies according to the complexity with which the blades, airfoil characteristics, and induced velocity are accounted for. The most sophisticated strip theories take into account the pitch control and aeroelastic blade motions, and those additional effects on instantaneous blade section angles of attack [66]. None presently include substantial modeling of 3-dimensional (e.g., spanwise flow) effects, and none include significant estimation of unsteady aerodynamics. These simulations are normally used to estimate steady loads and performance first, and are then used to build rotor specifications based on estimated perturbations to the equilibrium conditions.

Some example results can be seen in Figure A-3. These were generated by a strip theory using reference 2-D airfoil data and the Goldstein approximation to the vortex. wake and tip effects [67]. The results are generally more accurate than the momentum theory, and generally come close to the average measured performance and loads of wind turbines, with the crucial exceptions of performance in stall and in transients. It 
FIGURE A-8. PERFORMANCE OF EXAMPLE PITCH CONTROL ROTOR

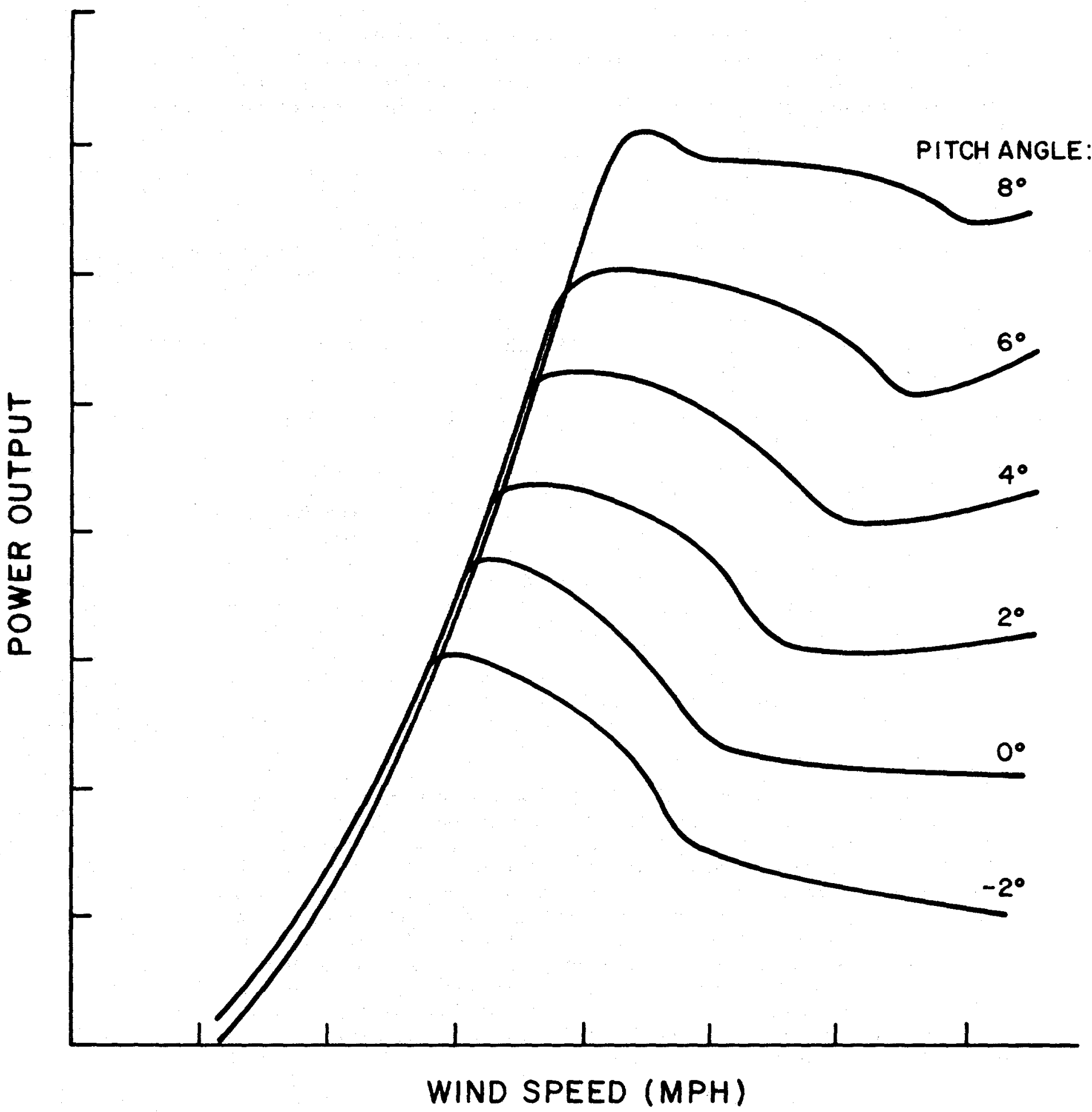


should be pointed out that the nondimensional coefficients shown in the figures are independent of rotor diameter, rotor speed, and wind speed, and thus depict the physics of both large and small rotors.

The various rotor aerodynamic flow states can be identified on the graphs. The Zero Slip Case is represented by $C Q=0$, at tip speed ratio (TSR) $=11.5$, and the Propeller State is below the abscissa, where CQ is negative (must provide power to the rotor). If the rotor is slowed down slightly, the angle of attack increases and the output power and thrust increase. The point where power is a maximum, i.e., [torque $\mathrm{X}$ RPM] is a maximum, occurs where the induced velocity is about $1 / 3$. The ideal momentum theory $\mathrm{CP}$ at this point is 0.5926 which is sometimes called the Betz limit for wind turbine power coefficient; in this example graph the actual $\mathrm{CP}$ is about 0.45 at a tip speed ratio of about 7 .

If angle of attack is increased beyond this by decreasing RPM further, the inflow increases on the blade until the airfoil stalls. Power and thrust fall off, power falling faster.

\section{Dynamic Stall}

This Section presents a short description of dynamic stall, from "Analysis of the Development of Dynamic Stall Based on Oscillating Airfoil Experiments", by Carr, McAlister, and McCroskey, NASA-TN-D-8382, January 1977 [9].

"Dynamic stall is a phenomenon associated with an airfoil moving beyond its static stall angle while experiencing a rapid change in angle of attack. In steady flow, the angle of stall is essentially fixed for any given airfoil geometry; at most, it is a weak function of Reynolds number. However, when an airfoil is moved rapidly through an angle of attack range that includes the static stall angle, the angle of maximum lift can be greatly increased, and becomes strongly dependent on the rate and amplitude of oscillation. This dynamic overshoot of the static stall angle occurs with no detectable change in the loading trend until a strong vortex appears near the airfoil leading edge. The pitching moment is then radically altered [see Figure 7], beginning with a large negative pitching moment which occurs as the vortex moves over the airfoil; when the vortex leaves the airfoil, the lift abruptly drops. The flow over the airfoil then becomes quiescent for a portion of the oscillation cycle, with a fully developed separated wake region appearing. Flow separation will usually persist for the remainder of the cycle, thus causing large hysteresis loops to develop in both the lift and pitching moment curves when viewed as a function of angle of attack (see Figures A-4 [9] and A-5 [68])." 
FIGURE A-4. DYNAMIC STALL EVENTS ON A NACA 0012 AIRFOIL [9]

THE EVENTS OF

DYNAMIC STALL ON

THE NACA OOI2 AIRFOIL
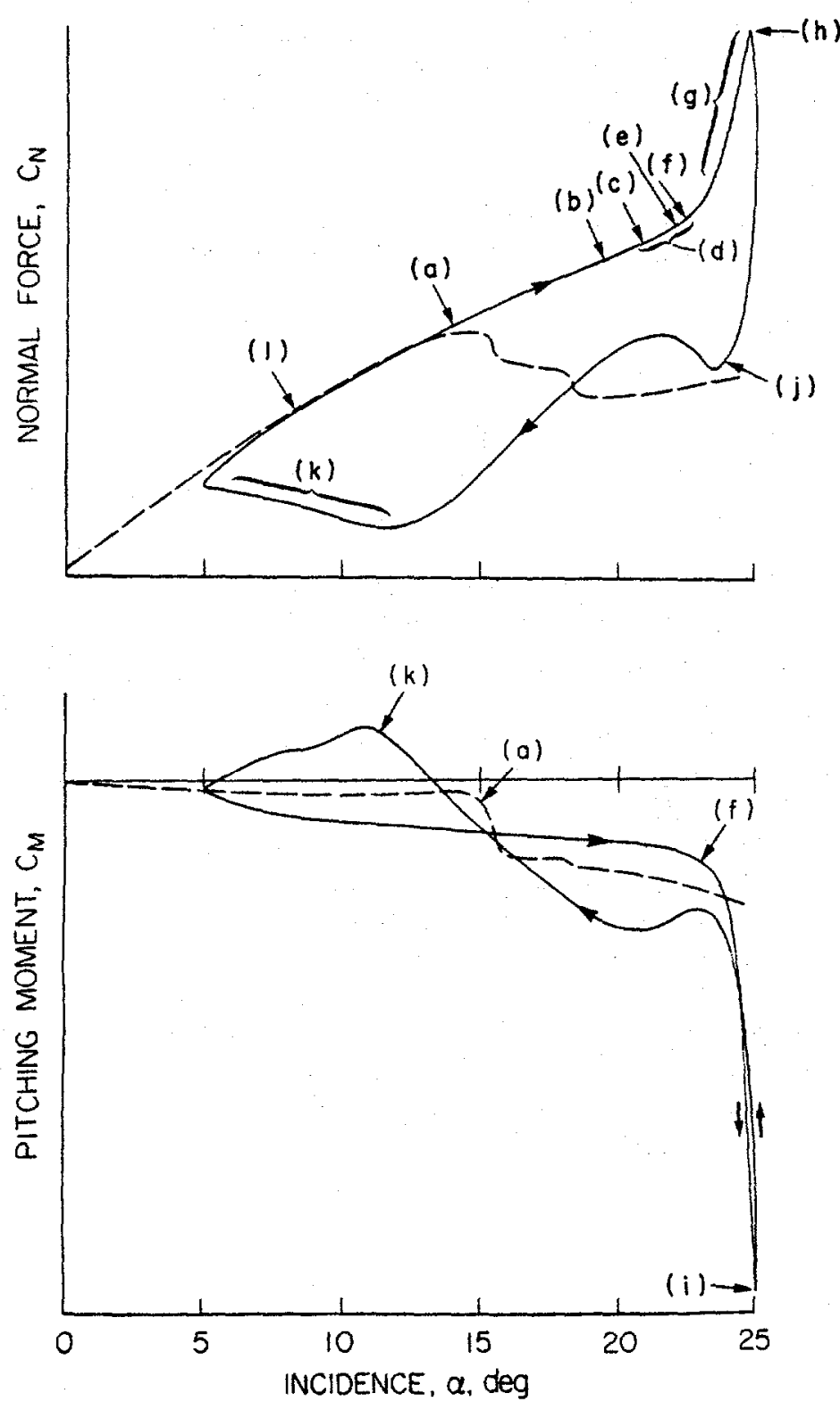

(a) STATIC STALL ANGLE EXCEEDED

(b) FIPST APPEARANCE OF FLOW REVERSAL ON SURFACE

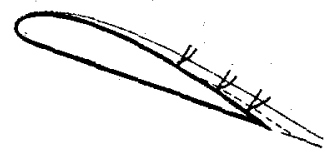

(c) LARGE EDDIES APPEAR IN BOUNDARY LAYER

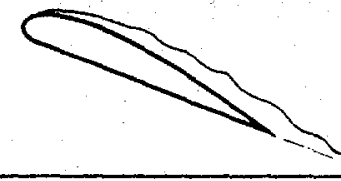

(d) FLOW REVERSAL SPREADS OVER MUCH OF AIRFOIL CHORD

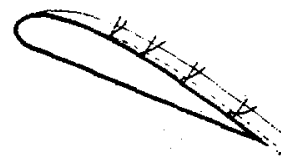

(e) VORTEX FORMS NEAR LEAOING EDGE

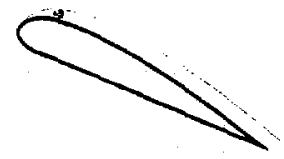

( $f$ ) LIFT SLOPE INCREASES

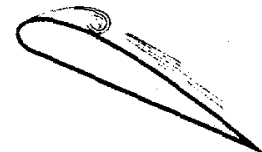

(g) MOMENT STALL OCCURS

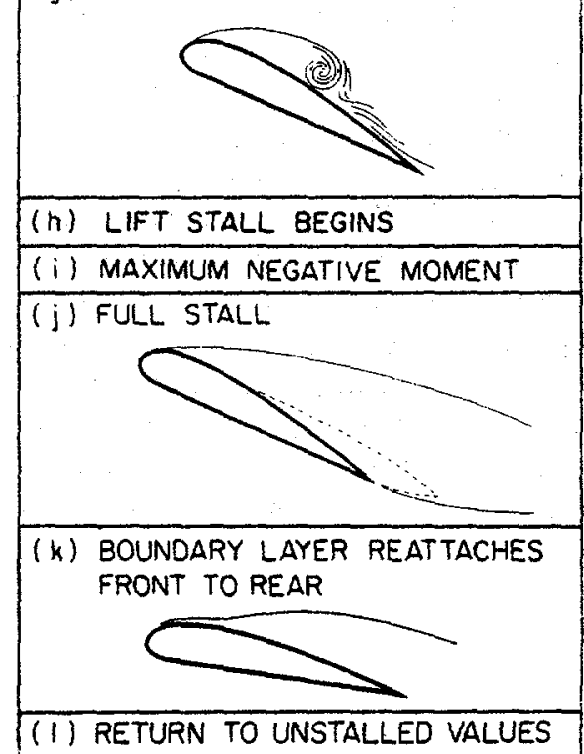


FIGURE A-5. DYNAMIC STALL FLOW EVENTS AND STREAMLINES [68]

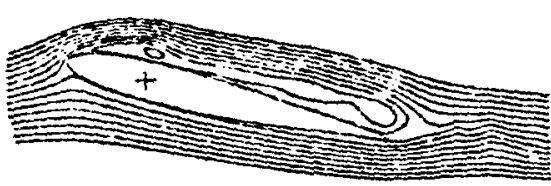

a) $\alpha=15.78^{\circ}$

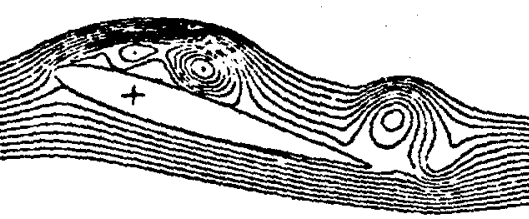

d) $\alpha=18.97^{\circ}$

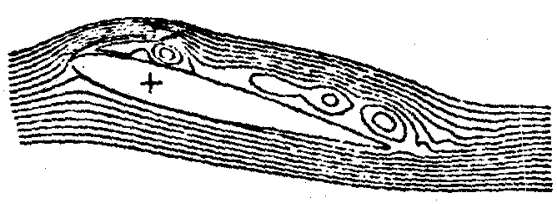

b) $\alpha=16.56^{\circ}$

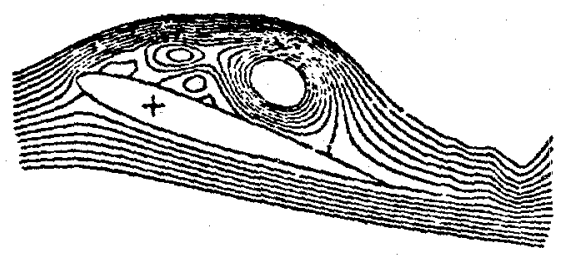

e) $\alpha=19.99^{\circ}$

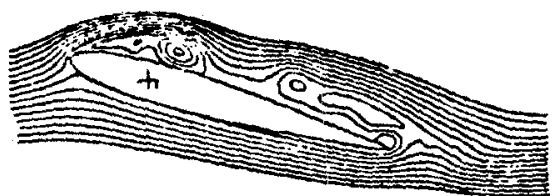

c) $\alpha=17.28^{\circ}$

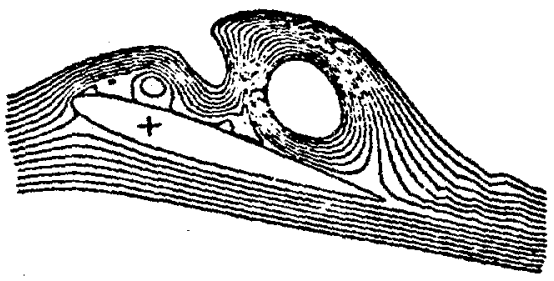

f) $\alpha=19.44^{\circ}$ 


\section{WIND TURBINE COMPARISON}

The rotor largely determines the performance, reliability, and especially weight of the rest of the system. This Section presents two examples in the 15-meter diameter class, of horizontal axis wind turbines which illustrate this. These turbines represent two production machines with similar cost and output. They should be viewed as two demonstrated design approaches to rotor aerodynamic design, even though they have very different deployment, operating, and development histories.

The example contrasts a high tip speed ratio approach with a low tip speed ratio approach. Both are stall-controlled rotors. The aerodynamic ranges of the airfoils on these two rotors are very different, as are the weights.

Table 1. Rotor Comparison

\begin{tabular}{|c|c|c|}
\hline Manufacturer & Storm Master & Bonus \\
\hline $\begin{array}{l}\text { Diameter } \\
\text { Number of blades } \\
\text { Design Speed } \\
\text { Tip Speed } \\
\text { Solidity } \\
\text { Optimum Tip Speed Ratio }\end{array}$ & $\begin{array}{l}40 \mathrm{ft} . \\
3 \\
133 \mathrm{RPM} \\
279 \mathrm{ft} / \mathrm{sec} \\
3.2 \% \\
9.5\end{array}$ & $\begin{array}{l}50 \mathrm{ft} \\
3 \\
50.3 \mathrm{RPM} \\
132 \mathrm{ft} / \mathrm{sec} \\
10 \% \\
5.5\end{array}$ \\
\hline $\begin{array}{l}\text { KW-design power output } \\
\text { Annual Productivity }\end{array}$ & $\begin{array}{r}50 @ 35 \mathrm{MPH} \\
125,000 \mathrm{KW}-\mathrm{h} / \mathrm{yr}\end{array}$ & $\begin{array}{r}55 @ 28 \mathrm{MPH} \\
140,000 \mathrm{KW}-\mathrm{h} / \mathrm{yr}\end{array}$ \\
\hline $\begin{array}{l}\text { Rotor Weight } \\
\text { Aloft Weight } \\
\text { Tower Weight } \\
\end{array}$ & $\begin{array}{r}420 \mathrm{lb} \\
2100 \mathrm{lb} \\
2900 \mathrm{lb}\end{array}$ & $\begin{array}{l}2645 \mathrm{lb} . \\
10748 \mathrm{lb} . \\
15211 \mathrm{lb} .\end{array}$ \\
\hline $\begin{array}{l}\text { Blade Mass Moment, I } \\
\text { Lock number } \\
\text { Airfoil } \\
\text { Flap frequency, } p \\
\text { Max. transient tip deflection }\end{array}$ & $\begin{array}{l}140 \text { slug-ft } \\
11.4 \\
\text { NACA } 4415 \\
1.05 \\
4.5 \mathrm{ft} \\
\end{array}$ & $\begin{array}{l}2573 \text { slug-ft } \\
5 \\
\text { NACA } 4415 \\
3.46 \\
0.5 \mathrm{ft} .\end{array}$ \\
\hline $\begin{array}{l}\text { Thrust@ rated } \\
\text { Blade loading@ rated } \\
\text { Disc loading@ rated } \\
\text { Gravity cyclic, \# cycles/yr }\end{array}$ & $\begin{array}{l}2000 \mathrm{lb} \\
49.7 \mathrm{lb} / \mathrm{ft}^{2} \\
1.6 \mathrm{lb} / \mathrm{ft}^{2} \\
40 \times 106\end{array}$ & $\begin{array}{r}2500 \mathrm{lb} \\
12.7 \mathrm{lb} / \mathrm{ft}^{2} \\
1.3 \mathrm{lb} / \mathrm{ft}^{2} \\
15 \times 106\end{array}$ \\
\hline
\end{tabular}




\section{Steady Aerodynamics}

In the previous discussion the free stream $V_{O}$ was constant and RPM was varied to change angle of attack. The two turbines of this example are constant speed, fixed-pitch designs. The angle of attack varies only with wind speed. Thus; the operating point on the nondimensional coefficient characteristic is determined by the wind speed alone. The CT and CP curves from the strip theory are given for these two rotors in Figure A-6.

At low wind speed, the tip speed ratio is high, so cut-in wind speed occurs at the $\mathrm{X}$-intercept where $\mathrm{CP}$ is just positive. This is very close to the Zero Slip Case, and as can be seen in the Bonus example, thrust coefficient is already high at this point owing to the flat blade angle. As wind speed increases, tip speed ratio decreases, angle of attack on the blades increases, and output power and thrust increase. The induced velocity is increasing from nearly zero at cut-in, to about $1 / 3$ at maximum $C P$.

Increasing wind speed further, increases angle of attack and induced velocity until stall is reached on some part of the blade. Actually the points of maximum CP for both the Bonus and Storm Master have substantial stall already on the inboard blade sections. With increased free stream $\mathrm{V}_{o}$ and deepening stall, comes Turbulent Wake State, and CP falls of $f$ rapidly, CT falling more slowly. Even though CT is falling, dimensional thrust is increasing due to the V-squared effect. As can be seen in the next curves, dimensional power actually stays about constant for both turbines, dropping off in a controlled way with increasing wind speed. This is precisely the design philosophy termed "stall control" for fixed-pitch wind turbines. It means the blades stall as tip speed ratio decreases, so output power is limited. Thrust, on the other hand, actually continues to increase for both these turbines.

The power curves are given in Figure A-7. These are just the dimensional plots of steady output power vs. steady wind speed. The power curve is the most significant comparator at present in the wind turbine industry since ideal yearly income is found by simply integrating this characteristic over the expected site annual wind speed distribution. The Method of Bins experimental technique is widely used at present in the industry to obtain the power curve.

As can be seen in the curves, both turbines continue to produce close to rated power in extremely high winds, by design. The rotors have actually become "drag devices" with very low power coefficients.

The point of maximum CP is also seen to occur at about $1 / 2$ of the range between cut-in and rated speed. This is accomplished by deliberate sizing of the drivetrain and generator, since some analytical studies have shown optimum productivity for such a power curve characteristic when integrated with an ideal wind speed distribution. Variations in this optimum load matching point are now known to be significantly dependent on the site average wind conditions: a high wind site having a large installed power to rotor area ratio, and a low wind site just the reverse. 
FIGURE A-6. ROTOR COMPARISON: THRUST AND POWER COEFFICIENT
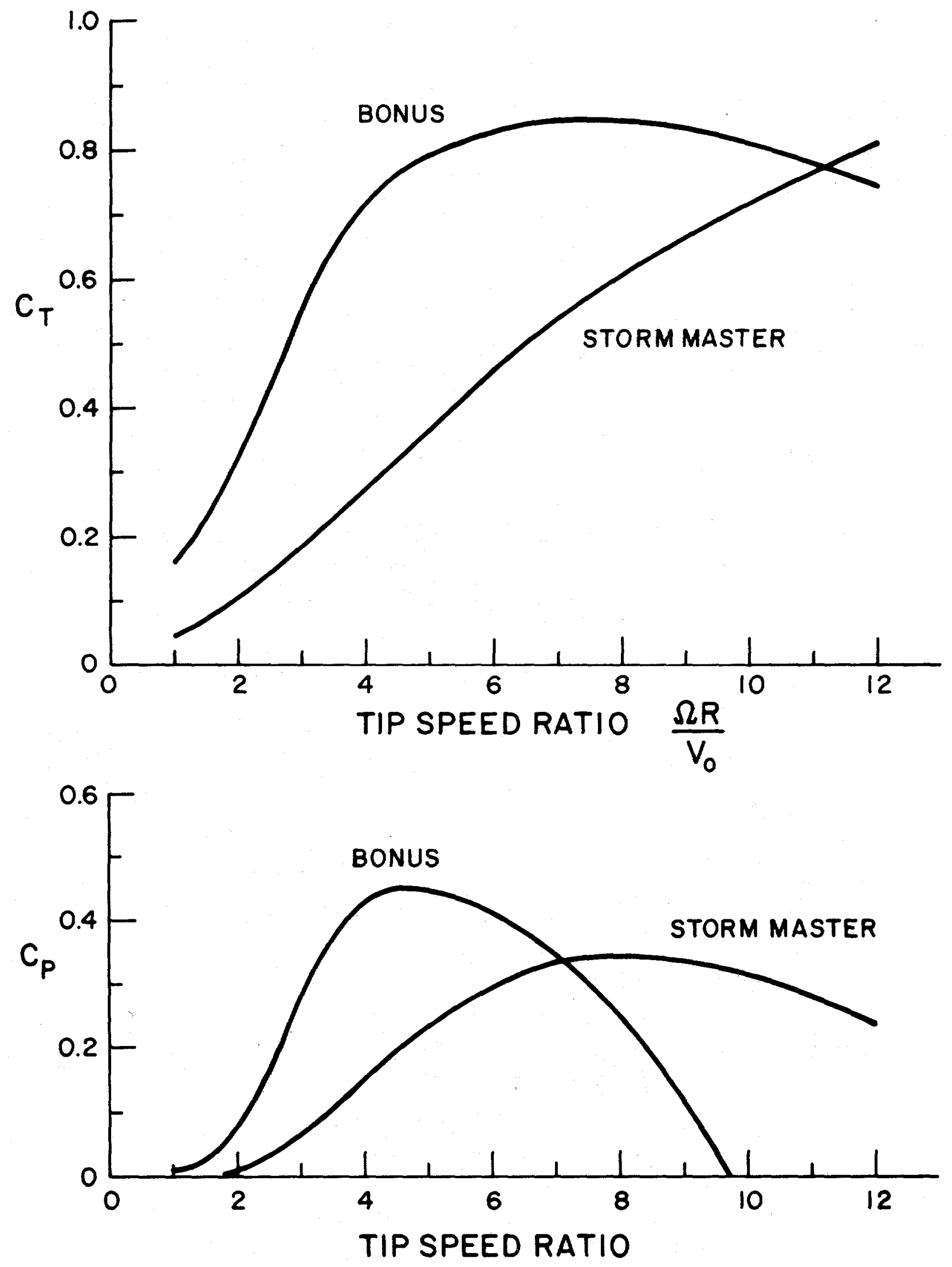
FIGURE A-7. ROTOR COMPARISON: POWER CURVES
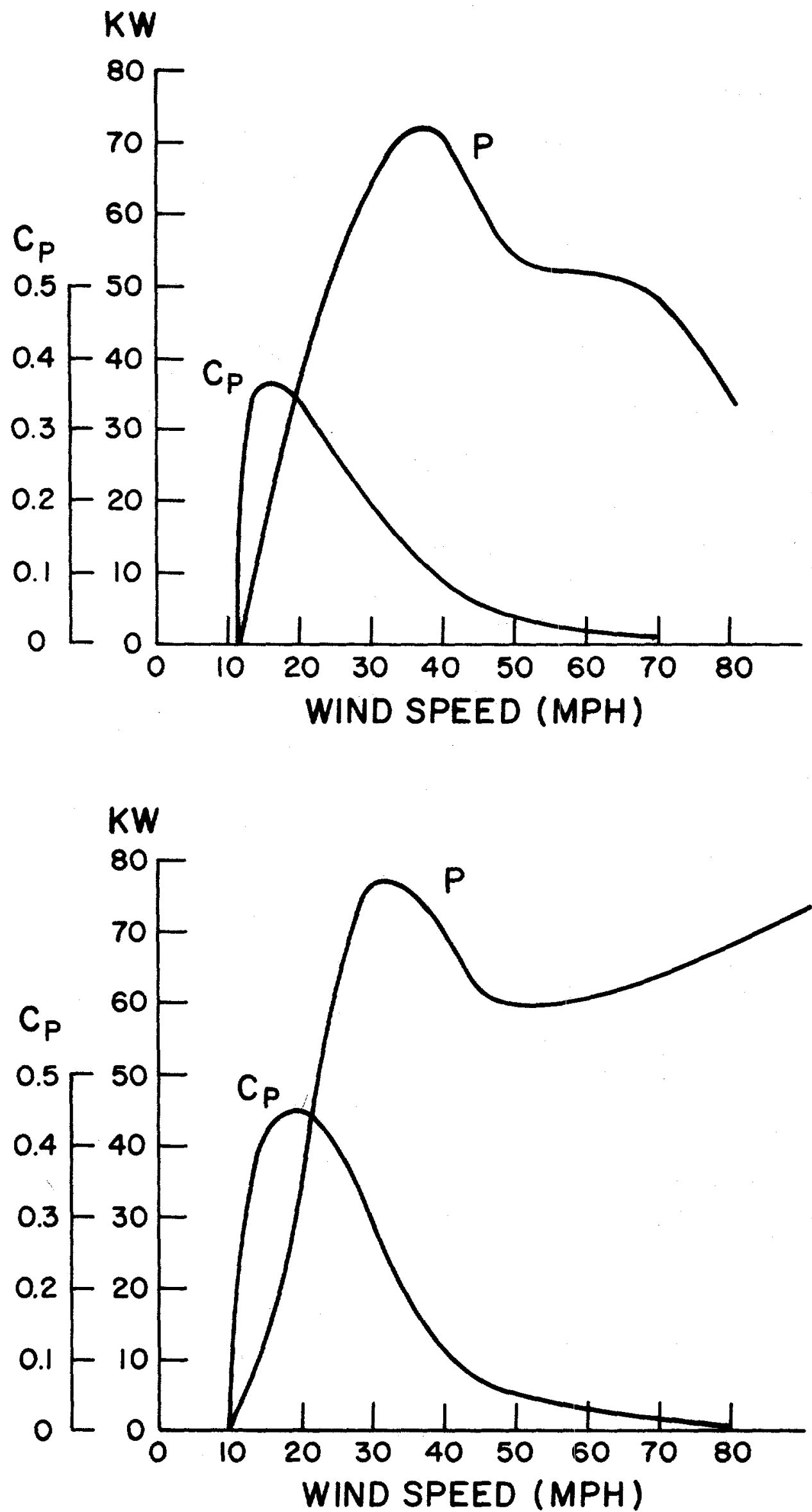


\section{Unsteady Aerodynamics}

For the two example rotors the following table is given for the 3/4-span airfoil section at rated conditions. A single inflow gust is superimposed: a $1 / 4$-second gust from 30 to $40 \mathrm{MPH}$.

Table 2. Airfoil Comparison: $r / R=0.75$

Storm Master

Bonus

Chord

Local speed (Omega X r)

Airfoil type

Blade loading

\author{
$0.667 \mathrm{ft}$. \\ $210 \mathrm{ft} / \mathrm{sec}$ \\ NACA 4415 \\ $50 \mathrm{lb} / \mathrm{ft}^{2}$ \\ or $75 \mathrm{lb} /$ span $\mathrm{ft}$.
}

$2.2 \mathrm{ft}$.

$99 \mathrm{ft} / \mathrm{sec}$

NACA 4415

$12.7 \mathrm{lb} / \mathrm{ft}^{2}$

or $5.8 \mathrm{lb} /$ span $\mathrm{ft}$.
Lift coefficient
1.20
.20
Angle of attack
$8 \mathrm{deg}$.
$-2 \mathrm{deg}$.

Due to sharp-edged gust: (1/4 sec gust from 30 to $40 \mathrm{MPH}$ )

\begin{tabular}{lcc} 
Delta angle of attack & $4 \mathrm{deg}$. & $8.4 \mathrm{deg}$. \\
Rate of change of alpha & $16 \mathrm{deg} / \mathrm{sec}$ & $33 \mathrm{deg} / \mathrm{sec}$ \\
Reduced frequency & .001 & .012 \\
\hline
\end{tabular}

Same gust effect at $r / R=0.25$ span station inboard:
Delta angle of attack
12 deg.
Rate of change
$48 \mathrm{deg} / \mathrm{sec}$
Reduced frequency
.008
$25 \mathrm{deg}$.
$100 \mathrm{deg} / \mathrm{sec}$
.116

The reduced frequency is a nondimensional measure of the time to convect a perturbation across the airfoil from leading to trailing edge. A value of 1.0 indicates a pitch excursion event, [d/dt (alpha) $X$ chord], which has the same period as the local section stream convection, [(rotor speed) $\mathrm{X}$ (span station radius)]. A reduced frequency of 0.1 or greater, coupled with high angle of attack, or high $\mathrm{CL}$, indicates dynamic stall.

As can be seen in Table 2 above, the Bonus wind turbine is susceptible to dynamic stall on the inboard sections for the example axial gust. The high solidity, low-RPM approach reduces the free stream convection to the point where this relatively common gust will cause unsteady lift effects on the inboard portion of blade. The integrated effect of this on blade loading and performance is still open for investigation.

\section{Fixed Pitch vs. Variable Pitch}

The above example dealt with relatively small turbines, of the 15-meter diameter class. These were fixed-pitch, stall-controlled, constant RPM turbines, and the aerodynamic variations and perturbations were caused by variation in the incident wind speed only. It is obvious that the fixed-pitch design, though simpler mechanically, has the great disadvantage of aerodynamic compromise: the pitch angle must be chosen for a single rotor flow 
condition, power coefficient, thrust coefficient, and blade angle of attack distribution. It follows from the plots of the last Section that the power coefficient is high at really only one wind speed. This necessity to compromise performance has led to blade geometric (e.g., twist and taper) design effort which results in "tip speed ratio-tolerant" rotor designs.

Simply stated, such designs have relatively high power coefficients over a wide range of tip speed ratios, which corresponds to the range expected at the intended site. The Bonus power curve is an example of this.

Another disadvantage of the fixed pitch approach is vulnerability. The fixed pitch rotor must always present its blades flatwise to high winds, gusts, and precipitation. Lastly, the fixed pitch compromise design point chosen for good performance at rated winds usually results in very poor static torque at low winds; that is, torque coefficient at startup is low or even negative, as is the case for fixed pitch vertical axis designs. This effect can be largely mitigated by large blade platform (solidity) and twist in the low tip speed ratio designs of smaller diameters.

For these reasons very large turbines have usually been pitch-controlled rather than stall-controlled. Some portion of the blade up to full span has pitching freedom up to the limit of full feather. The disadvantage of pitching freedom is complexity and associated cost. However, the pitch-control turbine can always be made to be self-starting and feathered (stable) in extreme conditions. The pitch-controlled machine also can directly alter blade angle of attack, thereby modulate pitch angle to vary the torque and thrust characteristics to obtain higher performance or lower vulnerability (see Figure A-8).

The steady aerodynamics of the pitch-controlled turbines are thus very different than for the fixed-pitch designs. However, the unsteady aerodynamics are much the same. Pitching mechanisms do not have the capability to "shed loads" because the control pitching rates are not fast enough. For example, the rotor of the graph (Figure A-8) is a 20-meter design, and the design pitching rate is only 8 degrees/second, which is an order of magnitude lower than the unsteady axial gust-induced rate of change in angle of attack. Therefore, the high frequency turbulence spectrum may cause very similar loading effects on both fixed- and variable-pitch rotors. 
FIGURE A-3. VARIOUS COEFFICIENTS VS. TIP SPEED RATIO
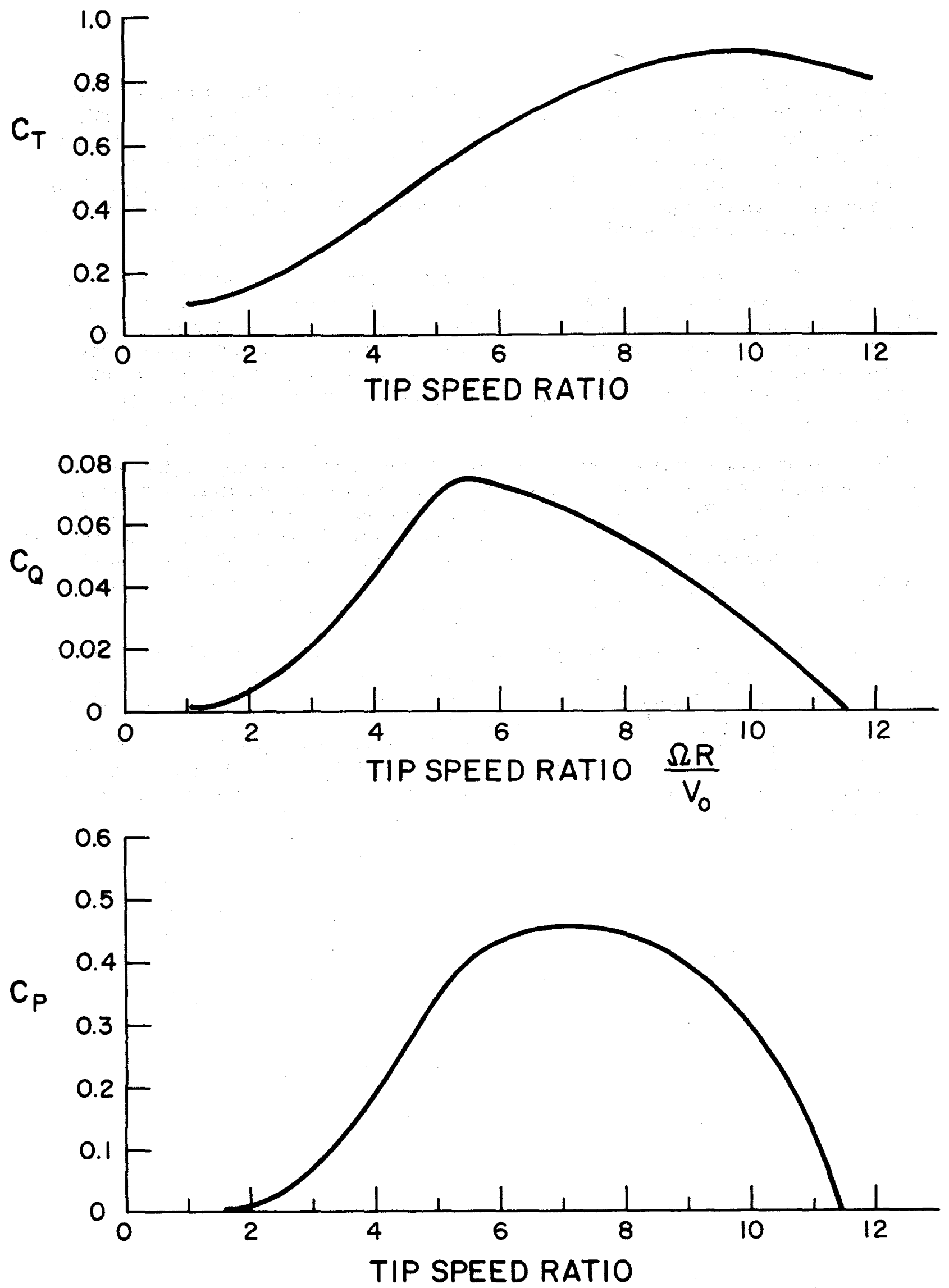


\section{APPENDIX 3. SUMMARY OF GENERIC DESIGN APPROACHES}

This Appendix presents the generic design approaches that were judged by the Panel to be most significant to continuing improvement in wind turbine aerodynamics. These approaches are somewhat indicative of specific hardware configurations, but the remarks are kept as general as possible so as not to be too specific to any certain mechanical design. The approaches, their requirements, characteristics, chief unknowns, known limitations, and possible improvements in performance and reliability, are summarized in Figure 2.

\section{STALL CONTROL}

The approach of stall-controlled rotors specifically means the use of normal airfoil stall, and its normal attenuation of blade lift, to limit load in the control region above rated wind speed. This approach uses fixed pitch and constant speed (RPM) to achieve stalling levels of angle of attack in high winds (see Figure A-7). The aerodynamic requirements for this approach are that the airfoil have a predictable and repeatable stalling characteristic, with a gentle transition between the unstalled and stalled operation. This indicates the need for a trailing edge stalling type airfoil, which normally has a thick airfoil section and large leading edge radius (see Figure 4). Additional characteristics are that the rotor operate at low R.PM and has large solidity. The thick airfoil sections and large leading edge radius require a large chord, and a low RPM is required to achieve high angles of attack in moderate winds.

The stall-controlled rotor thus sets up an aerodynamic condition where average lift coefficient is high at rated power, the load-limiting capability depends on passive airfoil stall, and large blade areas are exposed to extreme winds. Nevertheless, this type of rotor has been the most successful to date in the industry, due to the "boilerplate" nature of the rotor.

Currently, the size of stall-controlled rotors is thought to be limited by stall flutter [30], which is the inevitable result of the aerodynamic condition described above being combined with a blade stiffness high enough to cause large flapping velocities (see Figures A-9 and A-10). The historical solution is structural, and involves either making the blade stiffer (push the flutter boundary higher), or considerably less stiff (articulate or teeter the rotor). A more recently-proposed solution [32] is aerodynamic in which the airfoil is changed to have unique stalling characteristic not common to historical airfoils: the lift in this case does not fall after stall is reached, but stays practically constant, at the level of CL-max. This change removes the drop in CL-max that provides a steady-state limit cycle hysteresis, which is the stall flutter mechanism.

The chief unknowns in current design methodology for stall control are the prediction of unsteady airloads, prediction of the stalled behavior attainment of a repeatable transition through the stalling point, and assessment of the effect of roughness. 

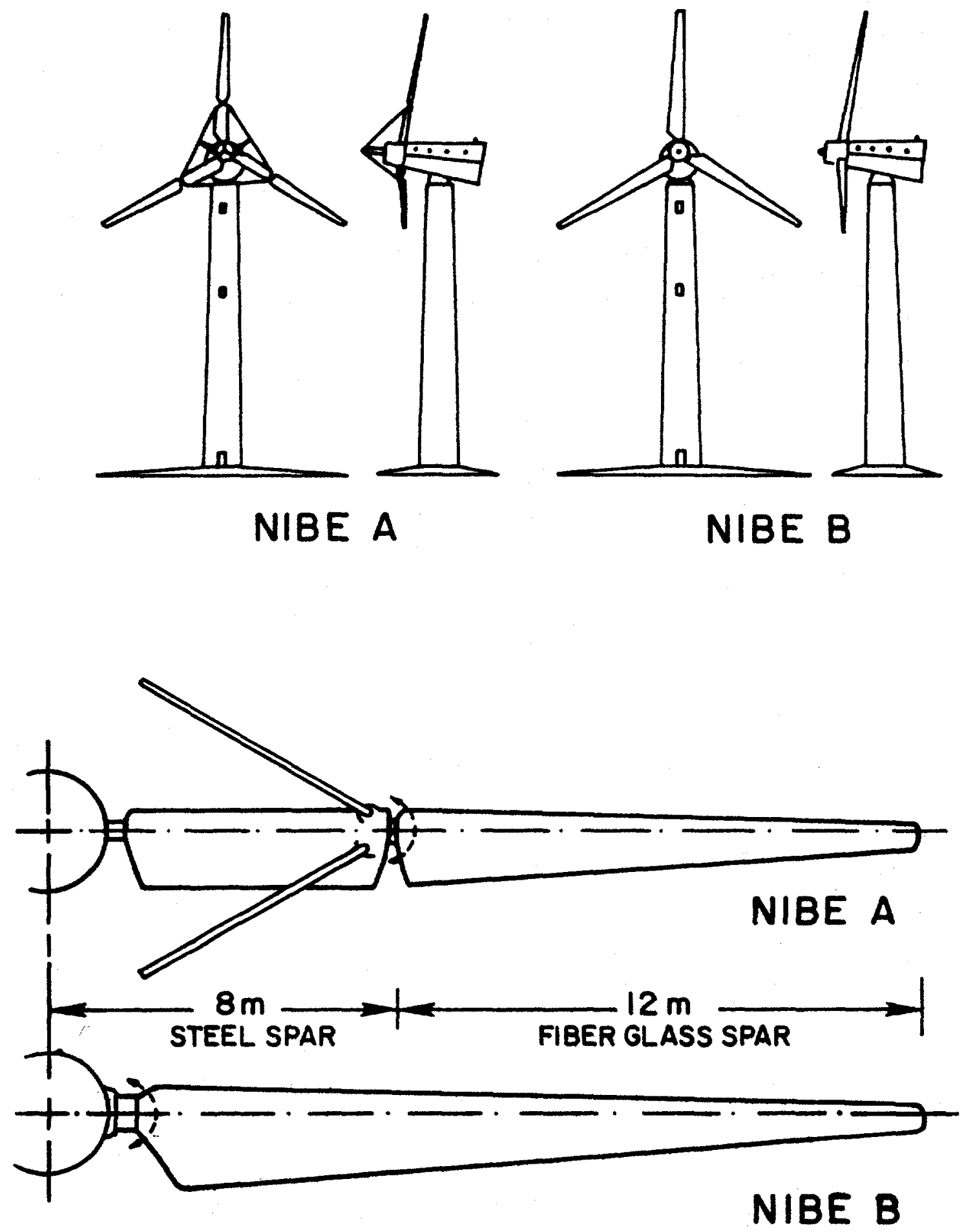

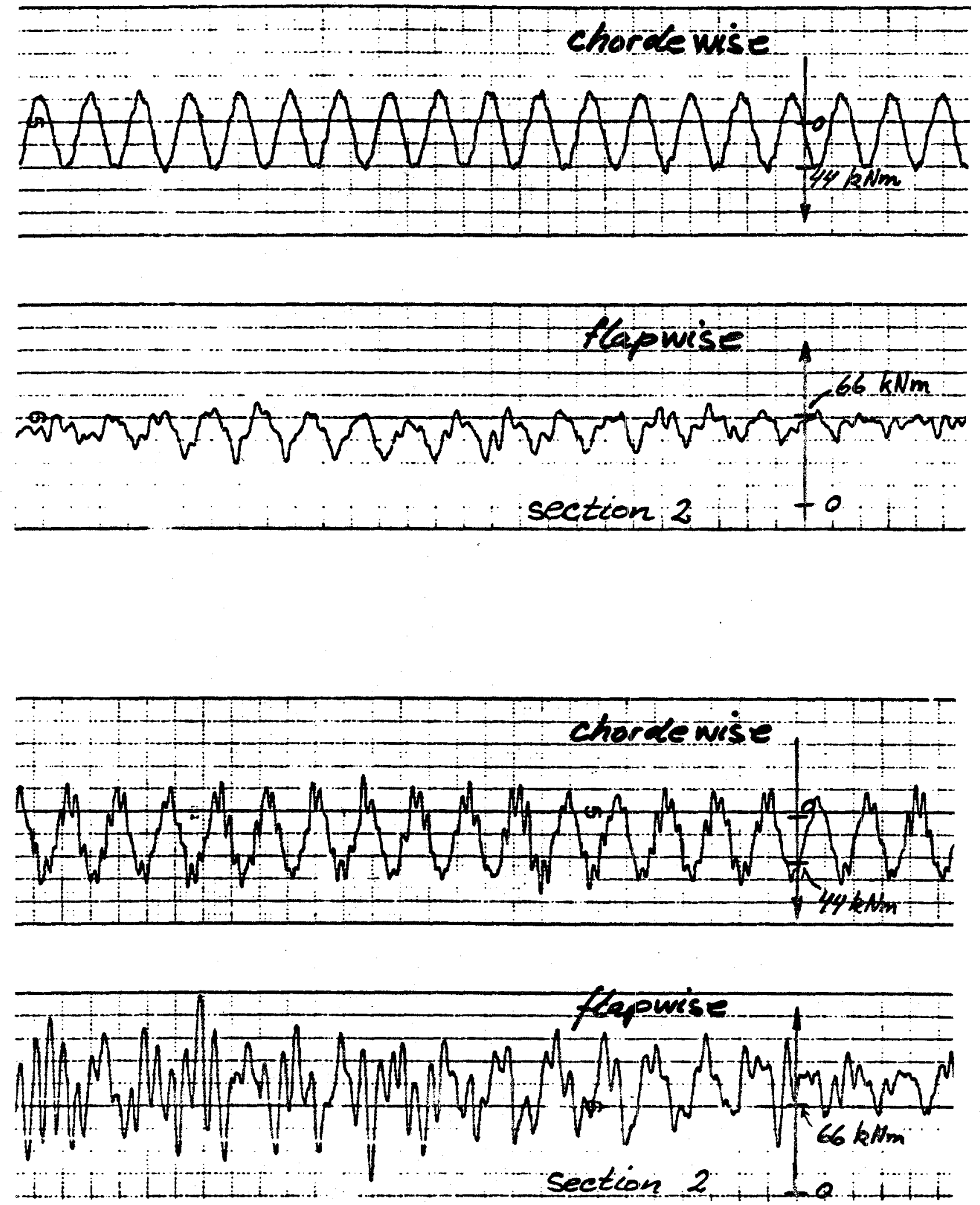

Nibe A Wind Turbine Chordwise and Flapwise Blade Bending

Moments During Normal Operation

and Stal1-Induced Vibrations

FIGURE A-10. NIBE A TURBINE: STALL-INDUCED VIBRATIONS [30] 
With proper effort expended on the unsteady aerodynamics and inflow as outlined in this Technical Assessment, about a $25 \%$ improvement in energy capture and significant further improvement in fatigue life are possible. This will be possible with a new unsteady aerodynamics technology base which will yield improved knowledge of the unsteady airloads and their fatigue effect, and provide the ability to reduce the weight, cost, and structure needed with improved unsteady airfoils.

\section{PITCH CONTROL}

Pitch-controlled rotors simply employ pitch angle changes to control loading. A pitch-controlled rotor can be used with constant speed or variable speed. The pitch degree of freedom requires a control system and algorithm (strategy), with many options for varying angle of attack in various control regions to improve performance. There is no compelling aerodynamic requirement that the airfoil stall gently, so a wider range of airfoils with higher performance and thinner sections can be used. The operating RPM as well is not constrained in order to produce high angles of attack, so the pitch-control machine can have a much higher operating tip speed and lower solidity.

The use of pitch-control allows another degree of freedom with which to extend the envelope of performance, and is thus not restricted to the load limit region. Pitch control can be used at any time when the average lift coefficient must be reduced. An example is the overspeed or shutdown control function (see Figure 17). The pitching degree of freedom is similar to the variable speed degree of freedom in that constant (average) angle of attack can be maintained on the blade (see Figure 26). The difference is in the unsteady behavior, which is driven by control limits in the former and rotor inertia in the latter.

The pitch-control machine is vulnerable to large transient airloads during pitch motions if the pitch rate is too high (see Figure A-11 [69]). In the load control region, the pitch control approach may result in higher transient gust loading since dynamic stall may force very high momentary lift coefficients, at a rate too high for pitch following.

Possible improvements to the pitch-controlled rotor are the same as for the stallcontrolled rotor. It is not likely that either one will be able to avoid dynamic stall entirely.

\section{VARIABLE SPEED}

The variable speed wind turbine can be either fixed or variable pitch, but must operate at constant tip speed ratio in the rising power region (control region 2). The requirement is that the driven load match the output torque as the square of RPM, and thus restrains the rotor to operate at constant tip speed ratio, where the RPM is linear with wind speed. Other variable speed options are possible, but the performance benefits are clearest with constant tip speed ratio operation. The turbine operates at its single best design point, or rotor efficiency, so the power coefficient is maximized (see Figure A-3). Load control is still necessary in the region above rated power, and this is achievable in various ways, such as pitch change and forced yaw. 


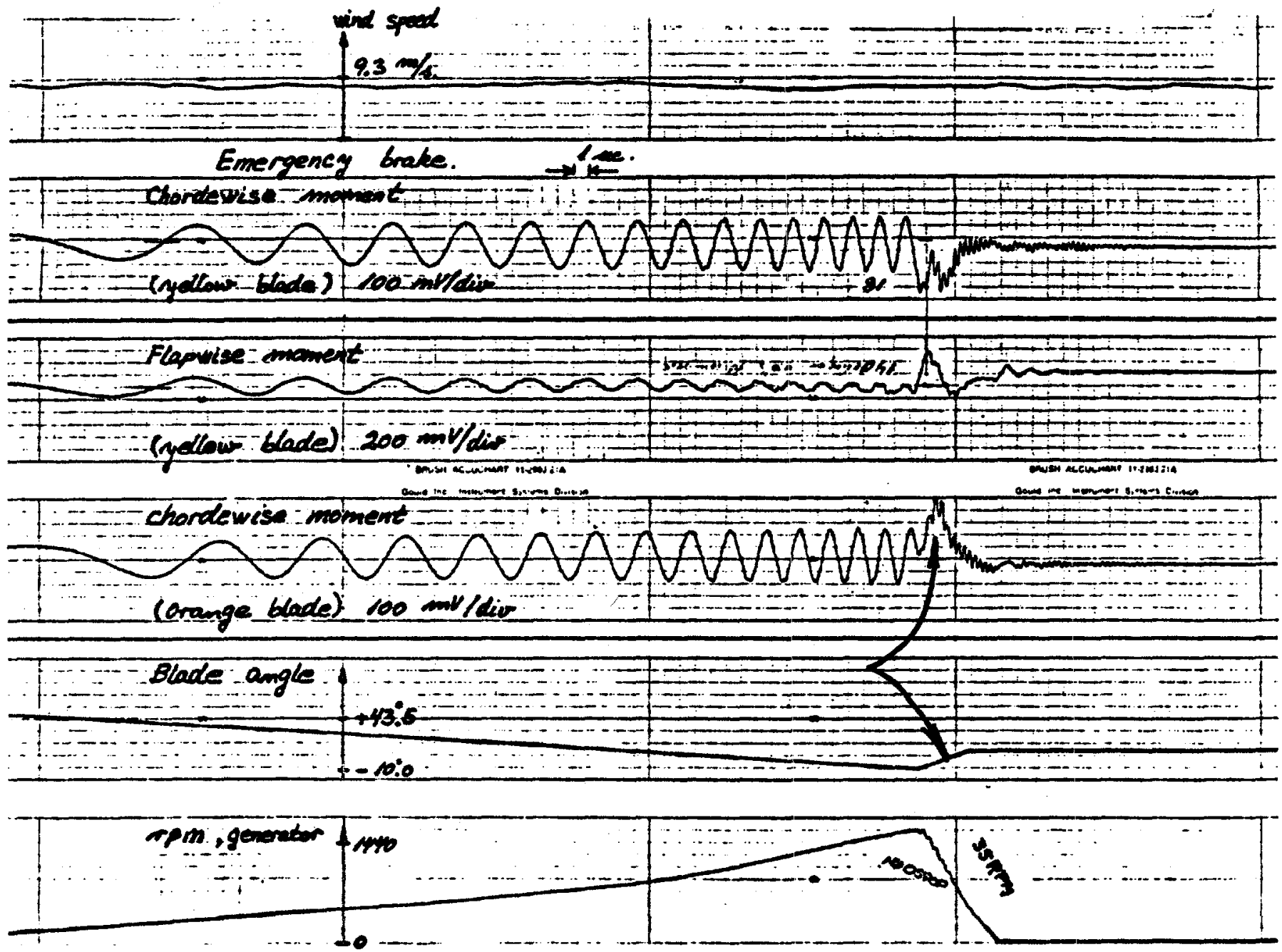

Nibe B Wind Turbine Root Bending Moments, Showing High Braking Transient Loads Due To High Pitching Rate of Blade Feathering 
One of the characteristics of constant tip speed ratio operation is that the rotor operates in load equilibrium, with the aerodynamic and inertial (centrifugal) loads in balance, and the average coning angle constant (see Chapter 7). This similarity condition holds for large rotors also, but the gravity loads do not scale, and this probably limits the large sizes [57]. Variable speed operation maintains constant (average) angle of attack on the rotor, and is comparable to pitch control in this way. Fluctuations about this equilibrium point are determined by the unsteady inflow and the rotor inertia.

Limitations of this approach consist mainly of the necessary driven load control. The only type of load which achieves this square-law torque matching exactly is a fluid mechanics device (as the rotor is also) such as a mechanical water pump or churn [70].

The possible improvements for a variable speed rotor, given the technology advances put forth in this Assessment, are similar to the stall- and pitch-control options.

\section{DARRIEUS (VAWT)}

The Darrieus is a specific design example in the category of vertical axis wind turbines. The blades are fixed pitch, rotate about a vertical axis at constant RPM, and have an approximately troposkein curved shape (see Figure A-12). Compared to HAWT of the same dimensions, the Darrieus has more blade area and weight but due to its larger "orbit" the rotor may influence a larger free streamtube. For this reason, it is not clear that the VAWT is restricted to the traditional Betz limit based on swept area for actuator performance, which is true for HAWT rotors without flow augmenters.

The Darrieus is independent of inflow direction changes, is a stall-control rotor, and operates at constant pitch, hence requires a separate shutdown system. The Darrieus airfoil can be said to never operate at steady conditions; the rotor is always in unsteady flow, and is vulnerable to all the unsteady effects possible throughout its life. This is both an advantage and a disadvantage, since the unsteady effects may be both.

Possible improvements in energy capture over present designs are estimated to amount to $50 \%$ given a well-performing unsteady airfoil. Possible improvements to airload calculation and fatigue life are at least as high as for HAWT rotors, and probably higher.

\section{STRAIGHT BLADE VAWT}

The straight bladed VAWT (Figure A-13) is another vertical axis approach which adds a possible degree of freedom in blade pitch as well as speed, blade geometry, and airfoil and traces a larger orbit than equivalent dimension HAWTs. It is also true for this approach that the Betz limit based on swept area may not apply, since the affected wind streamtube is unknown. It also operates exclusively in unsteady flow and is subject to both the advantages and disadvantages of dynamic stall.

The pitching freedom allows control options in the various regions to exploit dynamic stall. It may be possible to employ advantageous unsteady airfoils, with appropriate devices to tailor dynamic stall, and force them via the pitching system at 
FIGURE A-12. DARRIEUS WIND TURBINE ROTOR

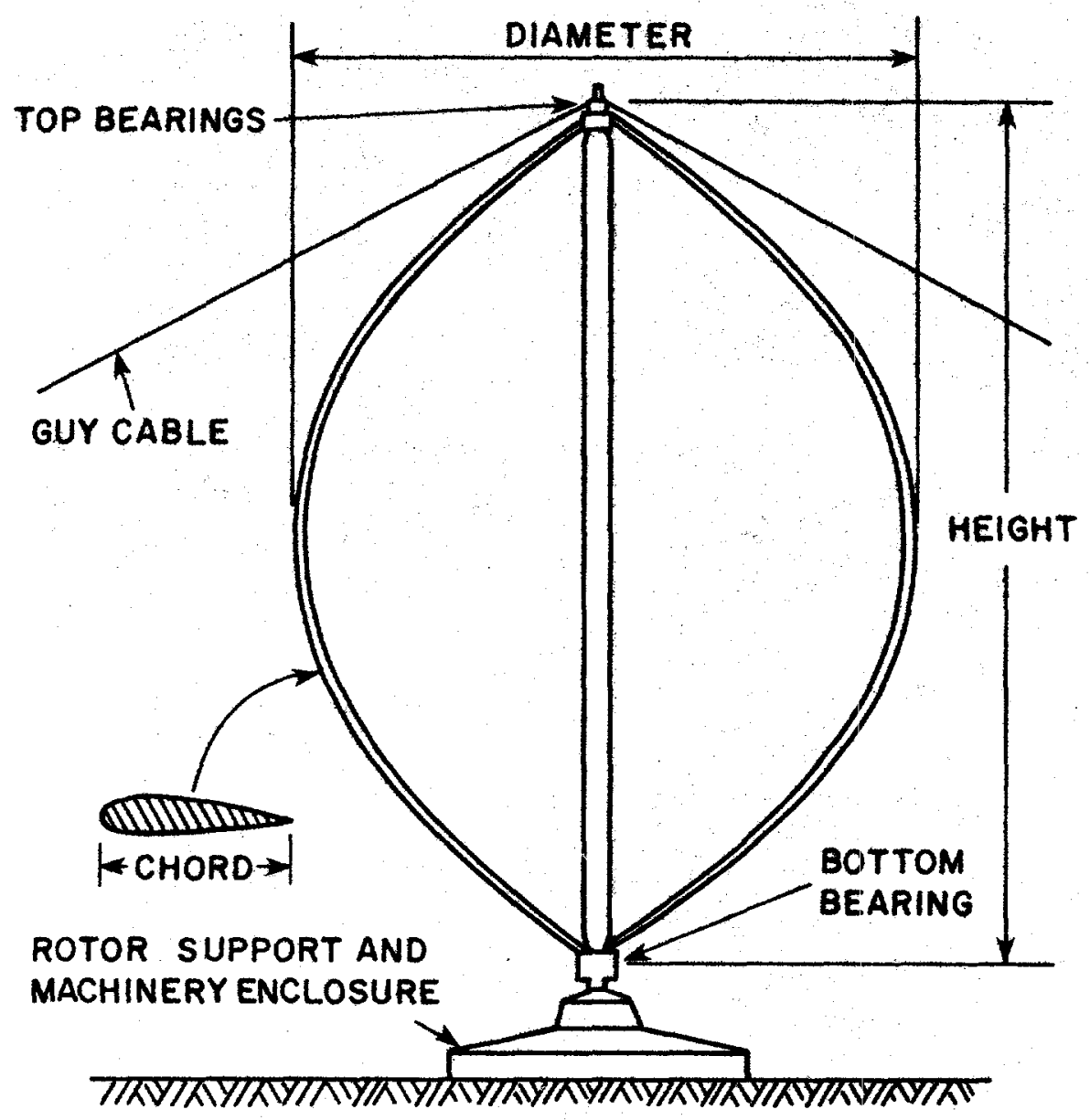


FIGURE A-13. STRAIGHT BLADE VERTICAL AXIS WIND TURBINE

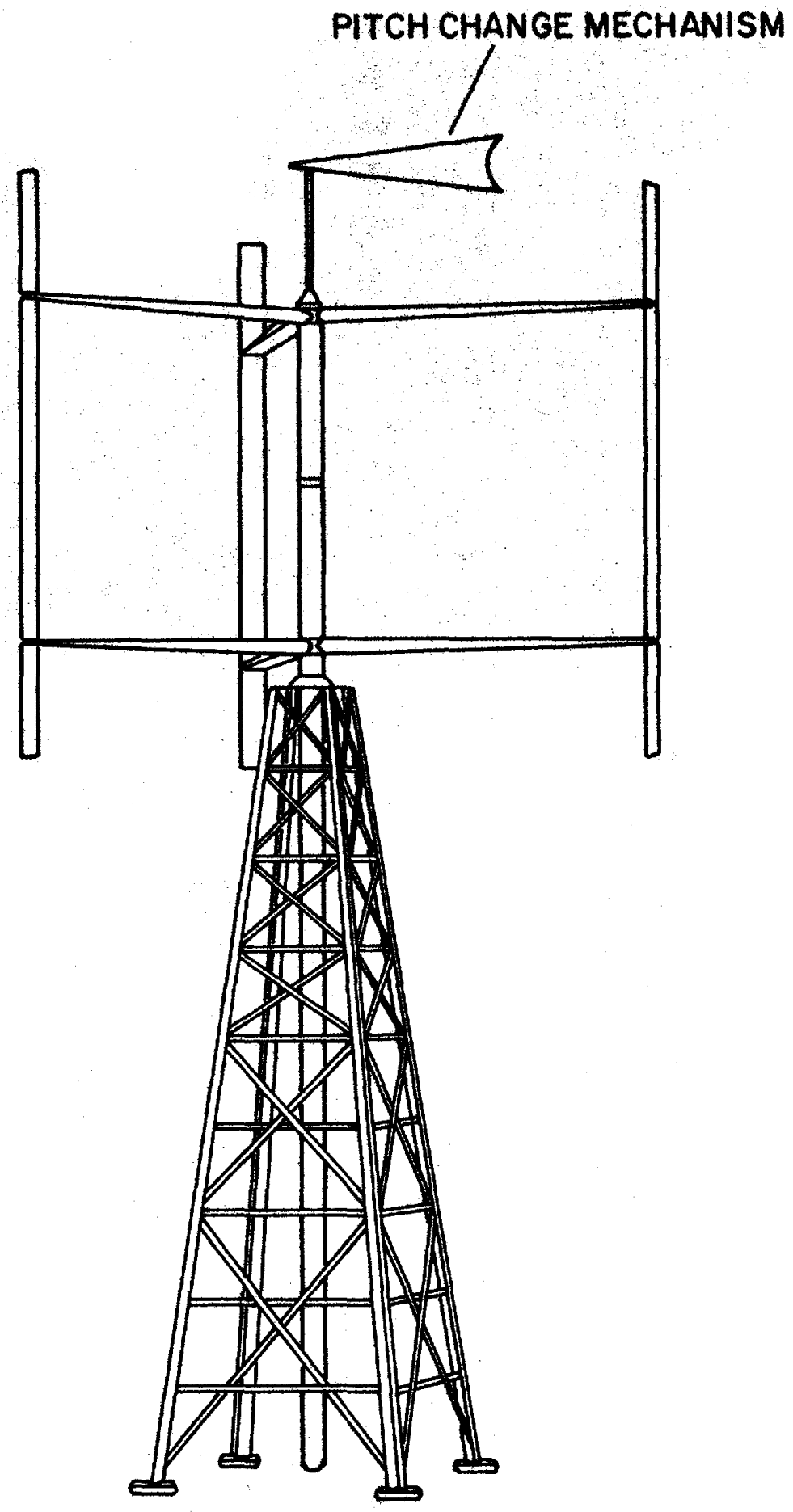


advantageous frequencies at various points on their orbits to convert their unsteady lift increases into torque without incurring a drag penalty. It is not hard to conceive of such a VAWT having much higher torque output than present values.

Advances in unsteady load prediction, as for the others, will also result in a dramatic improvement in rotor useful life.

\section{HIGH TIP SPEED RATIO}

The high tip speed ratio approach will likely be useful for rotors with very low solidity and large capture areas that will be used in low to moderate wind resource areas where the rotor capture are must be larger for a given installed power output. This approach will become more and more attractive as advances in understanding unsteady airloads and high strength-to-weight structures allow longer blades and lower rotor weights.

The high tip speed requires the characteristics of low solidity rotors: thin airfoils for low profile drag (which determines the upper tip speed limit for all rotors) and operation at low lift coefficients. The lower lift coefficient and higher speed allows airfoils to be used which have higher lift/drag ratios and (probably) better (since higher) Reynolds number performance. The tip speeds might be high enough that supercritical airfoils would be advantageous to delay drag rise at high subsonic Mach numbers. The limitation will be on acceptable noise levels (see Figure 28-30).

Another benefit of high tip speed ratio rotors is the smaller angle of attack change caused by inflow turbulence (see Figure 18). The high tip speed ratio turbine is less sensitive to unsteady aerodynamics effects for two reasons:

1. The rotor operates at lower angles of attack.

2. Inflow fluctuations induce smaller variation in angle of attack.

An increased burden is placed on aeroelastic analysis and the control functions due to the more stringent loads and dynamics requirements. Also, the thin airfoils likely to be used will probably be significantly more sensitive to roughness effects.

Possible improvements over present designs are dramatic. If this approach is possible, say, with advanced structures, energy capture could be increased by a factor of 2 to 3, which simply represents the larger rotor area which would be possible for the same cost or weight. Improvements in fatigue life are not as clear, since there is no technology base for comparison.

\section{FREE YAW (HAWT)}

The free yaw approach has the benefit of no active yaw sensors, actuators, or maintenance. The rotor for a free yaw turbine would by itself be stable in the unsteady inflow field, and have no average steady yaw tracking error. Present field experience indicates that active yaw systems are now, and will continue to be, high maintenance items [71], and that steady yaw tracking errors exist primarily due to the uncertainty of proper unsteady inflow measurement with the external sensors necessary [26]. 
The chief unknown in this approach is a detailed parametric knowledge of the aerodynamic yaw stability derivative, which is the restoring yawing moment. It is likely that dynamic stall plays a major role in determining this derivative for any set of unsteady conditions, just as for the unsteady airloads.

The possible improvement will be an increase in captured energy and a decrease in cyclic loading; both will result from better directional stability in unsteady flow. 
APPENDIX 4. WIND TURBINE INDUSTRY REPRESENTATIVES

AND SITES VISITED, JULY 1985

Wind Turbine Industry Briefing, Oakland, California, July 10-12, 1985:

Craig Hansen, University of Utah

Clint Coleman, North Wind Power Company

Jay Carter, Jr., Carter Wind Systems

Glidden Doman, Hamilton Standard

Herman Drees, FloWind Corporation

Jamie Chapman, U.S. Windpower, Inc.

Vaughn Nelson, West Texas State Univ.

Mike Zuteck, Consultant, Gougeon Bros.

M. C. Cheney, Windtech, Inc.

Wind Turbine Site Visits, California, July 12, 1985:

Boeing/PGE Mod-II Wind Turbine, Fairfield

U.S. Windpower, Altamont Pass

Altamont Energy Corp., Altamont Pass

Fayette Manufacturing Corp., Altamont Pass

Howden Wind Parks, Altamont Pass 